TRANSACTIONS OF THE

AMERICAN MATHEMATICAL SOCIETY

Volume 362, Number 1, January 2010, Pages 53-115

S 0002-9947(09)04940-X

Article electronically published on August 17, 2009

\title{
TOTALLY REAL IMMERSIONS OF SURFACES
}

\author{
ANDRZEJ DERDZINSKI AND TADEUSZ JANUSZKIEWICZ
}

\begin{abstract}
Totally real immersions $f$ of a closed real surface $\Sigma$ in an almost complex surface $M$ are completely classified, up to homotopy through totally real immersions, by suitably defined homotopy classes $\mathfrak{M}(f)$ of mappings from $\Sigma$ into a specific real 5-manifold $E(M)$, while $\mathfrak{M}(f)$ themselves are subject to a single cohomology constraint. This follows from Gromov's observation that totally real immersions satisfy the $h$-principle. For the receiving complex surfaces $\mathbf{C}^{2}, \mathbf{C P}^{1} \times \mathbf{C P}^{1}, \mathbf{C P}^{2}$ and $\mathbf{C P}^{2} \# m \overline{\mathbf{C P}^{2}}, m=1,2, \ldots, 7$, and all $\Sigma$ (or, $\mathrm{CP}^{2} \# 8 \overline{\mathbf{C P}}$ and all orientable $\Sigma$ ), we illustrate the above nonconstructive result with explicit examples of immersions realizing all possible equivalence classes. We also determine which equivalence classes contain totally real embeddings, and provide examples of such embeddings for all classes that contain them.
\end{abstract}

\section{CONTEnTs}

0 . Introduction 54

1. Preliminaries $\quad 55$

2. Statement of the results $\quad 57$

3. $\mathrm{A} \mathbf{Z}_{2}$ cohomology constraint $\quad 62$

4. Proof of Theorem $2.1 \quad 63$

5. Topological obstructions $\quad 64$

6. Degrees modulo $q$ for circle-valued mappings $\quad 65$

7. Proof of Theorem 2.2. Surjectivity $\quad 66$

8. Proof of Theorem 2.2, Injectivity 68

9. The simplest examples $\quad 70$

10. Zooming $\quad 71$

11. Immersions of spheres $\quad 72$

12. Totally real blow-ups $\quad 73$

13. Removability of complex points by blow-up $\quad 75$

14. Deformations involving complex points $\quad 76$

15. Other immersed two-spheres 78

16. Removal of transverse intersections $\quad 79$

17. Proof of Lemma 16.2

18. Connected sums 83

19. Proof of Theorem 18.1 87

20. Totally real tori and Klein bottles in $\mathbf{C}^{2}$

Received by the editors January 9, 2007.

2000 Mathematics Subject Classification. Primary 53C15, 53C42; Secondary 32Q60.

Key words and phrases. Totally real immersion, pseudoholomorphic immersion, Maslov invariant, $h$-principle.

The second author was partially supported by NSF grant no. DMS-0405825.

(C)2009 American Mathematical Society 53 
21. Proofs of Theorem 2.3 and Corollaries $2.4-2.9$

22. Obstructions for embedded orientable surfaces 91

23. Embeddings of nonorientable surfaces 92

24. The sets $\mathfrak{I}_{q}(\Sigma)$ and $\mathfrak{D}_{ \pm}^{\varepsilon}(M)$ in special cases

25. Closed surfaces and cohomology 94

26. More on tori and Klein bottles in $\mathbf{C}^{2} \quad 96$

27. The integer $q$ in (1.4) 97

28. Index and degree after modifications 98

29. Totally real Klein bottles in $\mathbf{C P}^{1} \times \mathbf{C P}^{1}$

30. Surfaces immersed in $\mathbf{C P}^{2} \# m \overline{\mathbf{C P}^{2}} \quad 102$

31. Some special cases of Theorem 2.17 103

32. A Diophantine equation 104

33. Deformations of pseudoholomorphic immersions 106

34. Proof of Theorem 2.18 107

35. Proofs of Theorem 2.19 and Corollaries 2.20-2.21 108

36. The remaining cases of Theorem 2.17 109

Appendix. Spheres and tori in $\mathbf{C P}^{2} \# 9 \overline{\mathbf{C P}^{2}} \quad 112$

References 113

\section{INTRODUCTION}

Given an almost complex surface, that is, an almost complex manifold $M$ with $\operatorname{dim}_{\mathbf{R}} M=4$, we ask which closed real surfaces $\Sigma$ admit totally real immersions/embeddings $f$ in $M$, and how such $f$ can be classified up to the equivalence relation $\sim_{\text {tri }}$ of being homotopic through totally real immersions.

We study these questions using a two-pronged approach. First, our Theorems 2.1 and 2.2 provide an answer for totally real immersions. Theorem 2.2 states that, when $M$ is simply connected, the $\sim_{\text {tri }}$ equivalence class of a totally real immersion $f: \Sigma \rightarrow M$ is completely determined by the Maslov index $\boldsymbol{i}=\boldsymbol{i}(f)$ and degree $\boldsymbol{d}=\boldsymbol{d}(f)$ of $f$, which in turn form an arbitrary element $(\boldsymbol{i}, \boldsymbol{d})$ of a specific set depending on $M$ and $\Sigma$. Theorem 2.1 classifies such $\sim_{\text {tri }}$ equivalence classes for arbitrary $M$, using the Maslov invariant $\mathfrak{M}(f)$, valued in a certain set of homotopy classes of mappings. Our definition of $\mathfrak{M}(f)$ in Section 2 is based on that in 2 .

What makes Theorems 2.1 and 2.2 less than completely satisfactory is the reliance of their proofs on Gromov's observation [20, p. 192] that totally real immersions satisfy the $h$-principle; see also [8, p. 176]. Consequently, those proofs offer little information about how the immersions which are shown to exist might actually be constructed. In addition, the two theorems deal only with the case of totally real immersions, as opposed to embeddings.

To make up for such shortcomings, we devote most of this paper (starting from Section (9) to our second approach, which deals with totally real immersions and embeddings $\Sigma \rightarrow M$ of arbitrary closed real surfaces $\Sigma$ in the "model" simply connected complex surfaces

$$
\mathbf{C}^{2}, \mathbf{C P}^{2}, \mathbf{C P}^{1} \times \mathbf{C P}^{1} \text { and } \mathbf{C P}^{2} \# m \overline{\mathbf{C P}^{2}}, \quad m \geq 1,
$$

$\mathbf{C P}^{2} \# m \overline{\mathbf{C P}^{2}}$ being obtained by blowing up any set of $m$ points in $\mathbf{C P}^{2}$. For these $M$, we provide explicit answers to the questions stated in the first paragraph. We 
begin by settling the existence question for totally real immersions/embeddings in $M$. (Table 1 in Section 2 summarizes the results.) Next, we construct examples of totally real immersions realizing all possible index-degree pairs $(\boldsymbol{i}, \boldsymbol{d})$. When $m \leq 7$ in (0.11), or $m=8$ and $\Sigma$ is orientable, this also answers the question about embeddings: some of our examples are embeddings, while, as an intersection argument shows, none of the other pairs $(\boldsymbol{i}, \boldsymbol{d})$ comes from a totally real embedding.

The constructions in question are summarized in Theorems 2.16 and 2.17

In our second approach we make a point of not using Theorems 2.1] or 2.2. Instead, we derive the nonexistence results directly from obstructions involving either characteristic classes and cohomology operations (for totally real immersions) or intersection numbers (for totally real embeddings). Our existence assertions are in turn established by explicit constructions.

In the case where the real surface $\Sigma$ is orientable, the nonexistence conclusions just mentioned were originally due to Lai [22] and Eliashberg and Kharlamov [7].

To prove Theorem 2.17 for $\mathbf{C P}^{2} \# m \overline{\mathbf{C P}^{2}}$ with $m=6,7,8$ we study a situation where a totally real immersion/embedding $\Sigma \rightarrow M$ of a closed oriented real surface $\Sigma$ in an almost complex surface $M$ can be obtained by deforming its exact opposite, that is, a pseudoholomorphic immersion/embedding $f: \Sigma \rightarrow M$. This turns out to be possible if and only if the normal bundle $\nu$ of $f$ and the tangent bundle $\tau$ of $\Sigma$ are anti-isomorphic as complex line bundles (Theorem 2.18), while the required (small) deformation is achieved by moving in the direction of a section $\psi$ of $\nu$ such that $\bar{\partial} \psi \neq 0$ everywhere, $\bar{\partial}$ being a Cauchy-Riemann operator. Although less constructive than the other existence proofs in the second part of the paper, such a technique is still more explicit than a general $h$-principle argument: it requires only finding a section $\psi$ of $\nu$ such that $\bar{\partial} \psi$ trivializes the line bundle $\operatorname{Hom}_{\mathbf{C}}(\bar{\tau}, \nu)$ (assumed trivial to begin with).

Our interest in the subject was sparked by Forstnerič's paper [13. We chose to focus on (0.1) as the receiving complex surfaces and the relation $\sim_{\text {tri }}$. However, other compact simply connected complex surfaces and other equivalence relations have been studied as well.

For instance, Slapar [26] proves the existence of totally real embeddings of closed oriented real surfaces in $K 3$ surfaces, their one-point blow-ups, and $E(3)$ surfaces. Borrelli [6] applies the $h$-principle to equivalence classes of totally real embeddings under the relation of being homotopic through totally real embeddings. As shown by Fiedler [9], even for totally real embeddings $T^{2} \rightarrow \mathbf{C}^{2}$ this last relation is stronger than being isotopic. On the other hand, Forstnerič and Rosay [16, Forstnerič [14, and Gong [17, 18, studied relations based on biholomorphic equivalence. See also Forstnerič [15].

Much work has also been done on totally real embeddings of closed $n$-manifolds in $\mathbf{C}^{n}$, for any $n \geq 2$. See [1, 4, 15, 11, 21, 27, 30] and [31.

Totally real immersions/embeddings may be viewed as a generalization of Lagrangian immersions/embeddings [4]. A detailed presentation of both categories and the $h$-principle can be found in the monographs [20] and [8].

\section{Preliminaries}

By 'planes' and 'lines' we always mean vector spaces. All manifolds are of class $C^{\infty}$ and connected, except when explicitly stated otherwise. 
An almost complex manifold is a real manifold $M$ with an almost complex structure (a $C^{\infty}$ bundle morphism $J: T M \rightarrow T M$ such that $J^{2}=-$ Id). The tangent bundle $T M$ then becomes a complex vector bundle in which $J$ is the multiplication by $i$. We usually write $i v$ rather than $J v$ for $v \in T_{x} M$ and $x \in M$.

A real vector subspace $W$ of a complex vector space $V$ is said to be totally real if $W \cap i W=\{0\}$. A totally real immersion/embedding of a real manifold $\Sigma$ (with or without boundary) in an almost complex manifold $M$ is an immersion/embedding $f: \Sigma \rightarrow M$ such that the image of the differential $d f_{x}$ at any $x \in \Sigma$ is a totally real subspace of $T_{f(x)} M$. If $f$ is a totally real embedding, the image $f(\Sigma)$ is called a totally real submanifold of $M$. (See [30, 20, and Remark 1.3])

Given an almost complex manifold $M$ with $\operatorname{dim}_{\mathbf{C}} M=n$, we define $E^{+}(M)$ and $E(M)$ to be the unit circle bundles of the determinant bundle $\operatorname{det}_{\mathbf{C}} T M=[T M]^{\wedge n}$ and, respectively, of its square $\left[\operatorname{det}_{\mathbf{C}} T M\right]^{\otimes 2}$. Thus, $E(M)$ is the $\mathbf{R P}^{1}$ bundle over $M$ associated with $\operatorname{det}_{\mathbf{C}} T M$. Both $E=E(M)$ and $E=E^{+}(M)$ are the total spaces of principal $\mathrm{U}(1)$-bundles over $M$, leading to the homotopy exact sequences

$$
\pi_{2} E \stackrel{\text { injective }}{\longrightarrow} \pi_{2} M \stackrel{\text { connecting }}{\longrightarrow} \pi_{1}[\mathrm{U}(1)]=\mathbf{Z} \longrightarrow \pi_{1} E \stackrel{\text { onto }}{\longrightarrow} \pi_{1} M .
$$

One also has an obvious twofold covering projection

$$
E^{+}(M) \rightarrow E(M)=E^{+}(M) / \mathbf{Z}_{2},
$$

equivariant relative to the homomorphism $\mathrm{U}(1) \ni z \mapsto z^{2} \in \mathrm{U}(1)$. Thus,

$$
\pi_{1}\left[E^{+}(M)\right] \subset \pi_{1}[E(M)], \quad \pi_{1}[E(M)] / \pi_{1}\left[E^{+}(M)\right]=\mathbf{Z}_{2} .
$$

If, in addition, $M$ is simply connected, (1.3) and the exactness of (1.1) give

$$
\pi_{1}[E(M)]=\mathbf{Z}_{q} \text { for some } q \in\{2,4, \ldots, \infty\} \text {, where we set } \mathbf{Z}_{\infty}=\mathbf{Z} \text {. }
$$

(More on $q$ can be found in Section 27,) For any manifold $\Sigma$ and any Abelian group $G$, we have natural isomorphic identifications

$$
H^{1}(\Sigma, G)=\operatorname{Hom}\left(\pi_{1} \Sigma, G\right)=\operatorname{Hom}\left(H_{1}(\Sigma, \mathbf{Z}), G\right) .
$$

Thus, according to (1.5) with $G=\mathbf{Z}_{2}$,

$$
w_{1}(\Sigma) \in H^{1}\left(\Sigma, \mathbf{Z}_{2}\right) \text { is the orientation homomorphism } \pi_{1} \Sigma \rightarrow \mathbf{Z}_{2} .
$$

We will also use Wu's formula 24, valid whenever $\Sigma$ is a closed real surface:

$$
w_{1}(\Sigma) \smile w_{1}(\Sigma)=[\chi(\Sigma) \bmod 2] \in H^{2}\left(\Sigma, \mathbf{Z}_{2}\right)=\mathbf{Z}_{2} .
$$

Remark 1.1. In terms of (1.5), the homomorphism $H^{1}(\Sigma, G) \rightarrow H^{1}\left(\Sigma, G^{\prime}\right)$ of coefficient reduction, corresponding to a homomorphism $h: G \rightarrow G^{\prime}$ of Abelian groups, sends a homomorphism $\varphi: H_{1}(\Sigma, \mathbf{Z}) \rightarrow G$ to $h \circ \varphi$.

Remark 1.2. The following properties of closed real surfaces $\Sigma$ are both well known and easily derived from Remark 25.1 and (25.3) i) in Section 25] the torsion subgroup of $H_{1}(\Sigma, \mathbf{Z})$ is trivial when $\Sigma$ is orientable, and isomorphic to $\mathbf{Z}_{2}$ otherwise; while, if $\chi(\Sigma)$ is odd, the torsion subgroup is not contained in the kernel of $w_{1}(\Sigma)$.

Remark 1.3. Our definition of a totally real subspace $W \subset V$ differs from that in [20, where $\operatorname{Span}_{\mathbf{C}} W$ is required to have the maximum possible dimension $\min (k, n)$, for $k=\operatorname{dim}_{\mathbf{R}} W$ and $n=\operatorname{dim}_{\mathbf{C}} V$. The two definitions agree if $k \leq n$, and are both devoid of content if $k \geq 2 n-1$ (as one of them then makes every subspace totally real, and the other allows no such subspace unless $n=k=1$ ); 
however, $k \leq n$ or $k \geq 2 n-1$ when $n=2$ (and $k \leq 2 n$ ), which is the case of our main interest.

\section{Statement of the Results}

Let $E(M)$ and $E^{+}(M)$ be as in Section 1 for an almost complex manifold $M$ with $\operatorname{dim}_{\mathbf{C}} M=n$. In view of (1.3), there exists a unique homomorphism

$$
\mathfrak{w}_{1}: \pi_{1}[E(M)] \rightarrow \mathbf{Z}_{2} \quad \text { with } \quad \operatorname{Ker} \mathfrak{w}_{1}=\pi_{1}\left[E^{+}(M)\right] .
$$

Thus (cf. (1.5)), $\mathfrak{w}_{1} \in H^{1}\left(E(M), \mathbf{Z}_{2}\right) \backslash\{0\}$ is the first Stiefel-Whitney class of the real line bundle over $E(M)$ associated with the $\mathbf{Z}_{2}$ bundle (1.2).

If $f: \Sigma \rightarrow M$ now is a totally real immersion of a real manifold $\Sigma$ in an almost complex manifold $M$ with $\operatorname{dim}_{\mathbf{R}} \Sigma=\operatorname{dim}_{\mathbf{C}} M=n$, we define the Maslov invariant $\mathfrak{M}(f)$ of $f$ to be the homotopy class of the mapping $\Sigma \rightarrow E(M)$ that sends $x \in \Sigma$ to the real line in $\left[T_{f(x)} M\right]^{\wedge n}$ spanned by the exterior product $v_{1} \wedge \ldots \wedge v_{n}$, where $v_{j}=d f_{x} e_{j}$ for any basis $e_{1}, \ldots, e_{n}$ of $T_{x} \Sigma$. (See also Section 3 and [2].)

Obviously, $\mathfrak{M}(f)$ depends only on the $\sim_{\text {tri }}$ equivalence class of $f$, for $\sim_{\text {tri }}$ defined in the Introduction. The following result is obtained in Section 4 by a straightforward application of the $h$-principle:

Theorem 2.1. Given an almost complex surface $M$ and a closed real surface $\Sigma$, the assignment $f \mapsto \mathfrak{M}(f)$ establishes a one-to-one correspondence between the set of all $\sim_{\text {tri }}$ equivalence classes of totally real immersions $f: \Sigma \rightarrow M$ and the set of those homotopy classes of mappings $\Theta: \Sigma \rightarrow E(M)$ for which

$$
\Theta^{*} \mathfrak{w}_{1}=w_{1}(\Sigma) \text { in } H^{1}\left(\Sigma, \mathbf{Z}_{2}\right) \text {, with } \mathfrak{w}_{1} \in H^{1}\left(E(M), \mathbf{Z}_{2}\right) \text { as in (2.1). }
$$

If $M$ in Theorem 2.1 happens to be simply connected, $\mathfrak{M}(f)$ may be replaced by a pair of more tangible invariants: the Maslov index $\mathbf{i}(f)$ of a totally real immersion $f: \Sigma \rightarrow M$, and its degree $\boldsymbol{d}(f)$, described below.

Specifically, discussing mappings $f$ from a real $n$-manifold $\Sigma$ into an almost complex manifold $M$, we will assume that an orientation of $\Sigma$ has been selected, as long as one exists; that is, $\Sigma$ is either oriented, or nonorientable. We then define a group $\mathbf{Z}_{[2]}$ associated with $\Sigma$ by

$$
\mathbf{Z}_{[2]}=\mathbf{Z} \text { if } \Sigma \text { is oriented, } \mathbf{Z}_{[2]}=\mathbf{Z}_{2} \text { if } \Sigma \text { is not orientable. }
$$

Let $[\Sigma] \in H_{n}\left(\Sigma, \mathbf{Z}_{[2]}\right)$ be the fundamental homology class of $\Sigma$. We set

$$
\boldsymbol{d}(f)=f_{*}[\Sigma] \in H_{n}\left(M, \mathbf{Z}_{[2]}\right) \text { with } n=\operatorname{dim}_{\mathbf{R}} \Sigma \text { and } \mathbf{Z}_{[2]} \text { as in (2.3). }
$$

Given a totally real immersion $f$ of a real manifold $\Sigma$ in an almost complex manifold $M$ such that $\operatorname{dim}_{\mathbf{R}} \Sigma=\operatorname{dim}_{\mathbf{C}} M=n$, we define $\boldsymbol{i}(f)$ to be the homomorphism $\pi_{1} \Sigma \rightarrow \pi_{1}[E(M)]$ induced by $\mathfrak{M}(f)$. Thus (cf. (1.4), (1.5)),

$$
\mathbf{i}(f) \in H^{1}\left(\Sigma, \mathbf{Z}_{q}\right) .
$$

Rather than being arbitrary elements of the (co)homology groups in question, $\boldsymbol{i}(f)$ and $\boldsymbol{d}(f)$ are both confined to specific subsets. Namely (see Lemma 7.1(b)), for a totally real immersion $f$ of a closed real surface $\Sigma$ in a simply connected almost complex surface $M$, and $q$ as in (1.4),

$$
\boldsymbol{i}(f) \in \mathfrak{I}_{q}(\Sigma) \subset H^{1}\left(\Sigma, \mathbf{Z}_{q}\right) \text { and } \boldsymbol{d}(f) \in \mathfrak{D}_{ \pm}^{\varepsilon}(M) \subset H_{2}\left(M, \mathbf{Z}_{[2]}\right) .
$$

Here $\varepsilon$ and \pm are $\mathbf{Z}_{2}$-valued parameters, determined by $\Sigma$ as follows:

$$
\varepsilon=1 \text { if } \Sigma \text { is orientable, } \varepsilon=0 \text { if it is not, and }(-1)^{\chi(\Sigma)}= \pm 1 \text {, }
$$


while $\mathfrak{D}_{ \pm}^{0}(M) \subset H_{2}\left(M, \mathbf{Z}_{2}\right)$ and $\mathfrak{D}_{ \pm}^{1}(M) \subset H_{2}(M, \mathbf{Z})$ are defined by

$$
\begin{aligned}
\mathfrak{D}_{+}^{0}(M) & =\operatorname{Ker}\left[w_{2}(M)\right], & & \mathfrak{D}_{-}^{0}(M)=H_{2}\left(M, \mathbf{Z}_{2}\right) \backslash \operatorname{Ker}\left[w_{2}(M)\right], \\
\mathfrak{D}_{+}^{1}(M) & =\operatorname{Ker}\left[c_{1}(M)\right], & & \mathfrak{D}_{-}^{1}(M)=\varnothing,
\end{aligned}
$$

with $c_{1}(M): H_{2}(M, \mathbf{Z}) \rightarrow \mathbf{Z}$ and $w_{2}(M): H_{2}\left(M, \mathbf{Z}_{2}\right) \rightarrow \mathbf{Z}_{2}$. Therefore, $\mathfrak{D}_{ \pm}^{\varepsilon}(M)$, if nonempty, is a coset of a subgroup in $H_{2}\left(M, \mathbf{Z}_{2}\right)$ or $H_{2}(M, \mathbf{Z})$. Finally, the subset $\mathfrak{I}_{q}(\Sigma)$ of $H^{1}\left(\Sigma, \mathbf{Z}_{q}\right)$ is given by

$$
\mathfrak{I}_{q}(\Sigma)=\left\{\lambda \in H^{1}\left(\Sigma, \mathbf{Z}_{q}\right):[\lambda \bmod 2]=w_{1}(\Sigma)\right\},
$$

where $H^{1}\left(\Sigma, \mathbf{Z}_{q}\right) \ni \lambda \mapsto[\lambda \bmod 2] \in H^{1}\left(\Sigma, \mathbf{Z}_{2}\right)$ denotes the $\bmod 2$ reduction homomorphism corresponding to the unique nonzero homomorphism $\mathbf{Z}_{q} \rightarrow \mathbf{Z}_{2}$. (Recall that $q \in\{2,4,6, \ldots, \infty\}$ in (1.4).) In Sections 7 and 8 we establish the following theorem.

Theorem 2.2. Given a simply connected almost complex surface $M$ and a closed real surface $\Sigma$, the assignment $f \mapsto(\mathbf{i}(f), \boldsymbol{d}(f))$ defines a bijective correspondence between the set of $\sim_{\text {tri }}$ equivalence classes of totally real immersions $f: \Sigma \rightarrow M$ and a specific subset $\mathcal{Z}(\Sigma, M)$ of the Cartesian product $\mathfrak{I}_{q}(\Sigma) \times \mathfrak{D}_{ \pm}^{\varepsilon}(M)$, in the notation of (1.4) and (2.7)-(2.9) .

The set $\mathcal{Z}(\Sigma, M)$ coincides with $\mathfrak{I}_{q}(\Sigma) \times \mathfrak{D}_{ \pm}^{\varepsilon}(M)$ except in the case where, simultaneously, $\Sigma$ is nonorientable and $\chi(\Sigma)$ is even, while $q$ is finite and divisible by 4 . In this latter case, $\mathcal{Z}(\Sigma, M)$ has half of the (finite) number of elements of $\mathfrak{I}_{q}(\Sigma) \times \mathfrak{D}_{ \pm}^{\varepsilon}(M)$, and consists of those $(\boldsymbol{i}, \boldsymbol{d}) \in \mathfrak{I}_{q}(\Sigma) \times \mathfrak{D}_{ \pm}^{\varepsilon}(M)$ which satisfy the following condition: if the image of the unique torsion element in $H_{1}(\Sigma, \mathbf{Z})$ under $\boldsymbol{i}: H_{1}(\Sigma, \mathbf{Z}) \rightarrow \mathbf{Z}_{q}$ is zero (or, nonzero), then $\boldsymbol{d} \in H_{2}\left(M, \mathbf{Z}_{2}\right)$ is (or, respectively, is not) the mod 2 reduction of an element of $\operatorname{Ker}\left[c_{1}(M)\right]$. (Cf. (1.5) and Remark [1.2.)

In contrast with Theorems 2.1 and 2.2 which make use of the $h$-principle, all the results listed below are established by explicit geometric arguments (even though one could also derive them from the $h$-principle). First, in Section 21 we prove the following six statements.

Theorem 2.3. Let $M$ be any almost complex surface. The class of closed real surfaces $\Sigma$ admitting a totally real embedding (or, immersion) in $M$ then includes the 2-torus $T^{2}$ and Klein bottle $K^{2}$ (and, for immersions, also the 2-sphere $S^{2}$ ), and is closed under the mapping $\Sigma \mapsto \Sigma \# T^{2} \# K^{2}$ (and, for immersions, under the connected-sum operation $\left.\left(\Sigma, \Sigma^{\prime}\right) \mapsto \Sigma \# \Sigma^{\prime}\right)$.

Corollary 2.4. Any closed real surface with an even Euler characteristic admits a totally real immersion in every almost complex surface.

Corollary 2.5. The 2-torus $T^{2}$ and all nonorientable closed real surfaces $\Sigma$ with $\chi(\Sigma) \equiv 0 \bmod 4$ admit totally real embeddings in every almost complex surface.

Corollary 2.6. Let $M$ be an almost complex surface. If there exists a totally real immersion $\mathbf{R P}^{2} \rightarrow M$, then every closed real surface $\Sigma$ admits a totally real immersion in $M$.

Corollary 2.7. Every closed real surface admits totally real immersions in $\mathbf{C P}^{2}$ and in all complex surfaces obtained from $\mathbf{C P}^{2}$ by blowing up any finite number of points. 
Corollary 2.8. The torus $T^{2}$, sphere $S^{2}$, and all nonorientable closed surfaces admit totally real embeddings in $\mathbf{C P}^{2} \# m \overline{\mathbf{C P}^{2}}$ for every integer $m \geq 2$.

Here and in the sequel, given a complex surface $M$, the connected sum $M \# s \overline{\mathbf{C P}^{2}}$ stands for any complex surface obtained by blowing up $s$ distinct points in $M$.

Corollaries 2.4 2.8 and Theorem 2.3 lead in turn to the following three results, the detailed proofs of which are given in Section 21 and Section 22 .

Corollary 2.9. For any almost complex surface $M$ which is a spin manifold, the closed real surfaces $\Sigma$ that admit totally real immersions in $M$ are precisely those having even Euler characteristics. This is, for instance, the case for $M=\mathbf{C}^{2}$ and $M=\mathrm{CP}^{1} \times \mathbf{C P}^{1}$.

Corollary 2.10. The class of closed real surfaces admitting totally real embeddings in $\mathbf{C P}^{1} \times \mathbf{C P}^{1}$ consists of the torus $T^{2}$, the sphere $S^{2}$, and all nonorientable closed surfaces with even Euler characteristics.

Corollary 2.11. For any fixed $m \in\{2,3, \ldots, 9\}$, the class of closed real surfaces admitting a totally real embedding in $\mathbf{C P}^{2} \# m \overline{\mathbf{C P}^{2}}$ consists of the torus $T^{2}$, the sphere $S^{2}$, and all nonorientable closed surfaces.

In each of the last three corollaries an existence statement based on an explicit elementary construction is coupled with a nonexistence assertion that uses elementary topological obstructions: an intersection-number relation (5.4) or (5.5), and a condition satisfied by either the first Chern class (for orientable surfaces $\Sigma$ ) or Stiefel-Whitney classes (for nonorientable $\Sigma$ ).

The Stiefel-Whitney class obstruction in (5.30) a) fails, however, to detect that some nonorientable closed surfaces $\Sigma$ do not admit totally real embeddings in $\mathbf{C}^{2}$, $\mathbf{C P}^{2}$ or $\mathbf{C P}^{2} \# \overline{\mathbf{C P}^{2}}$. Instead, following Audin 4] we use Massey's formula (23.1), which involves mod 4 intersection numbers and Pontryagin squares. This leads to a proof, in Section 23, of the next three corollaries; the first of them is a special case of a result of Audin [4. Corollaire 0.6], characterizing totally-real embeddability of closed $n$-manifolds in $\mathbf{C}^{n}$, for all even $n \geq 2$.

Corollary 2.12. A closed real surface $\Sigma$ admits a totally real embedding in $\mathbf{C}^{2}$ if and only if either $\Sigma$ is diffeomorphic to the torus $T^{2}$ or $\Sigma$ is nonorientable and $\chi(\Sigma) \equiv 0 \bmod 4$.

That $\chi(\Sigma) \equiv 0 \bmod 4$ for nonorientable $\Sigma$ in Corollary 2.12 is also obvious from the result of Whitney 33, according to which the Euler number of the normal bundle of $\Sigma$ (here equal to $\chi(\Sigma)$, by (5.1)) differs from $2 \chi(\Sigma)$ by a multiple of 4 whenever $\Sigma$ is a nonorientable closed real surface embedded in $\mathbf{C}^{2}$. Rudin 25] first showed that the Klein bottle admits a totally real embedding in $\mathbf{C}^{2}$.

Corollary 2.13. The class of closed real surfaces that admit a totally real embedding in $\mathbf{C P}^{2}$ consists of the torus $T^{2}$ and all nonorientable closed surfaces $\Sigma$ with $\chi(\Sigma) \equiv 0$ or $\chi(\Sigma) \equiv 1 \bmod 4$.

Corollary 2.14. The closed real surfaces admitting totally real embeddings in the complex surface $\mathbf{C P}^{2} \# \overline{\mathbf{C P}^{2}}$ are the torus $T^{2}$ and all nonorientable closed surfaces whose Euler characteristics are odd or divisible by 4. 
TABLE 1. Totally-real immersibility/embeddability of closed real surfaces $\Sigma$ in the complex surfaces (0.1) with $m \leq 9$. Here t.r. means 'totally real' and $\chi_{4} \in\{0,1,2,3\}$ stands for $\chi(\Sigma) \bmod 4$.

\begin{tabular}{|c|c|c|c|c|}
\hline the complex surface & $M=\mathbf{C P}^{2}$ & $M=\mathbf{C P}^{2} \# m \overline{\mathbf{C P}^{2}}$ & $M=\mathbf{C P}^{1} \times \mathbf{C P}^{1}$ & $M=\mathbf{C}^{2}$ \\
\hline $\begin{array}{c}\text { which orientable } \Sigma \text { are } \\
\text { t.r. embeddable in } M\end{array}$ & $T^{2}$ only & $\begin{array}{c}T^{2} \quad \text { only if } m=1 \\
S^{2}, T^{2} \text { if } 2 \leq m \leq 9\end{array}$ & $S^{2}, T^{2}$ only & $T^{2}$ only \\
\hline $\begin{array}{l}\text { t.r. embeddabil- } \\
\text { ity condition for } \\
\text { nonorientable } \Sigma\end{array}$ & $\chi_{4} \in\{0,1\}$ & $\begin{array}{c}\chi_{4} \neq 2 \text { if } m=1 \\
\text { all } \Sigma \text { t.r. embed- } \\
\text { dable if } 2 \leq m \leq 9\end{array}$ & $\chi(\Sigma)$ even & $\begin{array}{c}\chi(\Sigma) \\
\text { divisible } \\
\text { by } 4\end{array}$ \\
\hline $\begin{array}{l}\text { when a t.r. immer- } \\
\text { sion } \Sigma \rightarrow M \text { exists }\end{array}$ & \multicolumn{2}{|c|}{$\begin{array}{c}\text { exists for all } \Sigma \\
\text { (since it does for } \Sigma=\mathbf{R P}^{2} \text { ) }\end{array}$} & \multicolumn{2}{|c|}{$\begin{array}{c}\text { if and only if } \chi(\Sigma) \text { is even } \\
\text { (just because } M \text { is spin) }\end{array}$} \\
\hline
\end{tabular}

Corollaries 2.7 and 2.9-2.14 are summarized in Table1. For a conclusion similar to but weaker than Corollaries 2.13 and 2.14, see Proposition 5.1

Further such results can be derived from the following well-known existence theorem, proved in Section 14 via another explicit argument.

Theorem 2.15. Let $M^{\prime}$ be the complex surface obtained by blowing up $k$ distinct points, $k \geq 1$, in a given complex surface $M$. The class of closed real surfaces admitting a totally real embedding in $M^{\prime}$ then includes

(a) the 2-sphere $S^{2}$, if $k \geq 2$,

(b) the connected sum $\Sigma \# s \overline{\mathbf{R P}^{2}}$, whenever $s \in\{0,1, \ldots, k\}$ and $\Sigma$ is any totally real closed surface embedded in $M$ that contains at least $s$ of the $k$ blown-up points.

The next result, as stated, is a special case of Theorem 2.2 however, we establish it separately in Section 30, using - in contrast with our proof of Theorem 2.2 in Sections 7 and 8 - only explicit geometric constructions.

Theorem 2.16. If $M$ is one of the complex surfaces (0.1) and $\Sigma$ is a closed real surface, then $\mathcal{Z}(\Sigma, M)$ defined in the lines following Theorem 2.2 coincides with the set of all pairs $(\boldsymbol{i}, \boldsymbol{d})$ such that $\boldsymbol{i}(f)=\boldsymbol{i}$ and $\boldsymbol{d}(f)=\boldsymbol{d}$ for some totally real immersion $f: \Sigma \rightarrow M$.

Geometric constructions are also used to prove, in Section 36, a similar theorem about totally real embeddings. First, according to (5.5) and (23.2), we have the well-known relations

i) $\boldsymbol{d} \cdot \boldsymbol{d}=-\chi(\Sigma) \in \mathbf{Z}, \quad$ if $\Sigma$ is orientable,

ii) $\boldsymbol{d}^{2}=[\chi(\Sigma) \bmod 4] \in \mathbf{Z}_{4}$, if $\Sigma$ is not orientable.

Here $\boldsymbol{d}=\boldsymbol{d}(f)$ is the degree of any totally real embedding of a closed real surface $\Sigma$ in an almost complex surface $M$ and . denotes the intersection form in $H_{2}(M, \mathbf{Z})$. In (2.10) ii), $M$ is assumed, in addition, to be either diffeomorphic to $\mathbf{C}^{2}$ (and then $\boldsymbol{d}^{2}$ stands for $0 \in \mathbf{Z}_{4}$ ) or compact and simply connected (and then we set $\boldsymbol{d}^{2}=[\xi \smile \xi \bmod 4] \in H^{4}\left(M, \mathbf{Z}_{4}\right)=\mathbf{Z}_{4}$ for any $\xi \in H^{2}(M, \mathbf{Z})$ such that the mod 2 reduction of $\xi$ is the class $\mu \in H^{2}\left(M, \mathbf{Z}_{2}\right)$, Poincaré-dual to $\left.\boldsymbol{d} \in \mathrm{H}_{2}\left(M, \mathbf{Z}_{2}\right)\right)$.

Let $M$ be one of the complex surfaces (0.1). If $\Sigma$ is any closed real surface, the index-degree pairs $(\boldsymbol{i}, \boldsymbol{d})$ of totally real immersions $\Sigma \rightarrow M$ are precisely the elements of $\mathcal{Z}(\Sigma, M)$. (See Theorem 2.16 or Theorem 2.2.) That is no more the 
case when 'immersion' is replaced by 'embedding' since, as we just saw, (2.10) then must hold as well. For the surfaces (0.1) with $1 \leq m \leq 7$, condition (2.10) is, in fact, the only additional obstruction:

Theorem 2.17. Let $M$ be one of the complex surfaces (0.1) for $1 \leq m \leq 8$, and let $\Sigma$ be a closed real surface, orientable if $m=8$. Any $(\boldsymbol{i}, \boldsymbol{d})$ in the set $\mathcal{Z}(\Sigma, M)$ described immediately after Theorem 2.2, such that d satisfies (2.10), then equals $(\mathbf{i}(f), \boldsymbol{d}(f))$ for some totally real embedding $f: \Sigma \rightarrow M$.

Our proof of Theorem 2.17 for $\mathbf{C P}^{2} \# m \overline{\mathbf{C P}^{2}}$ with $m=6,7,8$ uses a fact, appearing below as Corollary 2.21 and obtained by deforming pseudoholomorphic immersions/embeddings to totally real ones (cf. the end of the Introduction). The last four results, stated next, are proved in Sections 34- 35.

Theorem 2.18. Given a pseudoholomorphic immersion/embedding $f$ of a closed oriented real surface $\Sigma$ in an almost complex surface $M$, the following three conditions are equivalent:

(a) the tangent bundle $\tau$ of $\Sigma$ and the normal bundle $\nu$ of $f$ are antiisomorphic as complex line bundles,

(b) $f^{*}\left[\operatorname{det}_{\mathbf{C}} T M\right]$ is trivial; that is, $f^{*} c_{1}(M)=0$ in $H^{2}(\Sigma, \mathbf{Z})$,

(c) $f$ is homotopic through immersions/embeddings $\Sigma \rightarrow M$ to a totally real immersion/embedding $f^{\prime}: \Sigma \rightarrow M$.

Moreover, $f^{\prime}$ in (c) then can be chosen arbitrarily $C^{1}$-close to $f$.

Here we call an immersion $f: \Sigma \rightarrow M$ pseudoholomorphic [20] if, for each $x \in \Sigma$, the image $d f_{x}\left(T_{x} \Sigma\right)$ is a complex line in $T_{f(x)} M$ and the isomorphism $d f_{x}: T_{x} \Sigma \rightarrow d f_{x}\left(T_{x} \Sigma\right)$ is orientation-preserving.

Let $M^{\prime}$ be the complex surface obtained from a given complex surface $M$ by blowing up any (ordered) $k$-tuple of distinct points. We will use the isomorphic identification

$$
H_{2}\left(M^{\prime}, \mathbf{Z}\right)=H_{2}(M, \mathbf{Z}) \times \mathbf{Z}^{k},
$$

with the convention that, for any oriented closed real surface $\Sigma$ embedded in $M^{\prime}$, the $\mathbf{Z}^{k}$ component of its homology class $[\Sigma]$ in the decomposition (2.11) consists of the ordered $k$-tuple formed by the intersection numbers of $\Sigma$ with the resulting $k$ exceptional divisors in $M^{\prime}$.

Theorem 2.19. Let $\Sigma$ be a one-dimensional compact complex submanifold of a complex surface $M$, and let $c$ be the integral of $c_{1}(M)$ over $\Sigma$. If $c \geq 0$ and $M^{\prime}$ is the complex surface obtained from $M$ by blowing up any $k$-tuple of distinct points, where $k \geq c$, then $\Sigma$ admits a totally real embedding $f$ in $M^{\prime}$ such that, under the identification (2.11),$f_{*}[\Sigma]=([\Sigma], 1, \ldots, 1,0, \ldots, 0)$ with $c \geq 0$ occurrences of 1 and $k-c \geq 0$ occurrences of 0 .

The identification $H_{2}\left(M^{\prime}, \mathbf{Z}\right)=\mathbf{Z}^{k+1}$ used in the next two corollaries is nothing other than (2.11) for $M=\mathbf{C P}^{2}$, with $H_{2}\left(\mathbf{C P}^{2}, \mathbf{Z}\right)=\mathbf{Z}$.

Theorem 2.19 has the following easy consequences (see Section [35):

Corollary 2.20. Given integers $d, k$ with $k \geq 3 d \geq 3$, let $M^{\prime}$ be the complex surface obtained from $\mathbf{C P}^{2}$ by blowing up any ordered set of $k$ points. Then the closed orientable surface $\Sigma$ of genus $(d-1)(d-2) / 2$ admits a totally real embedding $f: \Sigma \rightarrow M^{\prime}$ with $f_{*}[\Sigma]=(d, 1,1, \ldots, 1,0, \ldots, 0)$, where 1 occurs $3 d$ times and 0 occurs $k-3 d \geq 0$ times. 
Corollary 2.21. Given positive integers $d, j$ with $j \geq 2 d+2$, let $M$ be the complex surface obtained from $\mathbf{C P}^{2}$ by blowing up any $j$-tuple of distinct points. Then there exists a totally real oriented 2-sphere $\Sigma$ embedded in $M$ such that $[\Sigma]=(d, d-1,1, \ldots, 1,0, \ldots, 0)$, with 1 occurring $2 d+1$ times and 0 occurring $j-2 d-2$ times.

\section{A $\mathbf{Z}_{2}$ Cohomology constraint}

Given a complex vector space $V$ with $\operatorname{dim}_{\mathbf{C}} V=n \geq 1$, let $\mathrm{TR}(V)\left(\right.$ or $\left.\mathrm{TR}^{+}(V)\right)$ denote the set of all totally real (or, respectively, oriented totally real) vector subspaces of real dimension $n$ in $V$. (See Section 1) Also, let $\mathbf{R P}(W)$ (or $\mathrm{S}(W)$ ) be the real projective space (or sphere) of all real lines through 0 (or, respectively, rays emanating from 0 ) in any given real vector space $W$. We have natural mappings

$$
\mathfrak{L}: \operatorname{TR}(V) \rightarrow \operatorname{RP}\left(V^{\wedge n}\right), \quad \mathfrak{L}^{+}: \mathrm{TR}^{+}(V) \rightarrow \mathrm{S}\left(V^{\wedge n}\right)
$$

sending each $W$ in $\operatorname{TR}(V)$ or $\operatorname{TR}^{+}(V)$ to the real line/ray containing $e_{1} \wedge \ldots \wedge e_{n}$, where $e_{1}, \ldots, e_{n}$ is any basis (or positive-oriented basis) of $W$, and $V^{\wedge n}$ is the $n$th complex exterior power of $V$. Thus, $V^{\wedge n}$ is a complex line, while $\operatorname{RP}\left(V^{\wedge n}\right)$ and $\mathrm{S}\left(V^{\wedge n}\right)$ are circles. For a totally real immersion $f$ of a real manifold $\Sigma$ in an almost complex manifold $M$ with $\operatorname{dim}_{\mathbf{R}} \Sigma=\operatorname{dim}_{\mathbf{C}} M=n$, the Maslov invariant $\mathfrak{M}(f) \in[\Sigma, E(M)]$ was defined, in Section 2 to be the homotopy class of the mapping

$$
\Theta(f): \Sigma \rightarrow E(M)
$$

which sends each $x \in \Sigma$ to the real line $\mathfrak{L}\left(d f_{x}\left(T_{x} \Sigma\right)\right)$ in $\left[T_{f(x)} M\right]^{\wedge n}$, with $\mathfrak{L}$ as in (3.1) for $V=T_{f(x)} M$. If, in addition, $\Sigma$ is orientable, $\mathfrak{M}(f)$ can be lifted to $E^{+}(M)$; that is, there exists a homotopy class $\mathfrak{M}^{+}(f) \in\left[\Sigma, E^{+}(M)\right]$ whose composite with (1.2) is $\mathfrak{M}(f)$. A representative $\Theta^{+}(f): \Sigma \rightarrow E^{+}(M)$ of $\mathfrak{M}^{+}(f)$ is obtained by fixing an orientation of $\Sigma$, which does not affect the resulting homotopy class, and then assigning to any $x \in \Sigma$ the ray $\mathfrak{L}^{+}\left(d f_{x}\left(T_{x} \Sigma\right)\right)$ in $\left[T_{f(x)} M\right]^{\wedge n}$ (notation of (3.1)), with $d f_{x}\left(T_{x} \Sigma\right)$ oriented via $d f_{x}$.

Being a homotopy class of mappings, $\mathfrak{M}(f)$ induces a homomorphism

$$
[\Theta(f)]_{*}: \pi_{1} \Sigma \rightarrow \pi_{1}[E(M)]
$$

of the fundamental groups (with fixed base points). Under the identifications (1.4) and (1.5), the homomorphism (3.3) coincides with $\boldsymbol{i}(f)$ in (2.5).

We have the following easy fact.

Proposition 3.1. Let $f: \Sigma \rightarrow M$ be a totally real immersion of a real $n$-manifold $\Sigma$ in an almost complex manifold $M$ with $\operatorname{dim}_{\mathbf{C}} M=n$. Condition (2.2) then holds for the mapping $\Theta=\Theta(f)$ appearing in (3.2).

Proof. Let $\Gamma=\operatorname{Ker}\left[w_{1}(\Sigma)\right]$, so that $\Gamma$ is the subgroup of index 1 or 2 in $\pi_{1} \Sigma$ formed by all homotopy classes of loops $\gamma: S^{1} \rightarrow \Sigma$ for which $\gamma^{*}(T \Sigma)$ is orientable. Orientability of $\gamma^{*}(T \Sigma)$ means that the composite $\Theta(f) \circ \gamma$ can be lifted to a loop in $E^{+}(M)$; that is, its homotopy class lies in $\pi_{1}\left[E^{+}(M)\right]=\operatorname{Ker} \mathfrak{w}_{1}$ (see (2.1)). Thus, $\Gamma=\operatorname{Ker}\left(\mathfrak{w}_{1} \circ[\Theta(f)]_{*}\right)$. Hence $\mathfrak{w}_{1} \circ[\Theta(f)]_{*}=w_{1}(\Sigma)$, and so (1.5) gives (2.2) with $\Theta^{*}=[\mathfrak{M}(f)]^{*}$.

Remark 3.2. Let $\Sigma, M$ and $E(M)$ be a closed real manifold, an almost complex manifold and, respectively, the principal U(1)-bundle over $M$ defined in Section 1 . Furthermore, let $f: \Sigma \rightarrow M$ be a continuous mapping. 
(i) Continuous lifts $\Theta: \Sigma \rightarrow E(M)$ of $f$ satisfying (2.2) are nothing other than real-line subbundles of $f^{*}\left[\operatorname{det}_{\mathbf{C}} T M\right]$ isomorphic to $\operatorname{det}_{\mathbf{R}} T \Sigma$.

(ii) A lift as in (i) exists if and only if the complex line bundle $f^{*}\left[\operatorname{det}_{\mathbf{C}} T M\right]$ over $\Sigma$ is isomorphic to the complexification $\left[\operatorname{det}_{\mathbf{R}} T \Sigma\right]^{\mathbf{C}}$.

In fact, a lift $\Theta$ of $f$ to $E(M)$ selects a real line $\Theta_{x}$ in $\left[\operatorname{det}_{\mathbf{C}} T M\right]_{f(x)}$ for each $x \in \Sigma$, that is, forms a real-line subbundle of $f^{*}\left[\operatorname{det}_{\mathbf{C}} T M\right]$, while (2.2) states that this subbundle is isomorphic to $\operatorname{det}_{\mathbf{R}} T \Sigma$.

\section{Proof of Theorem 2.1}

Let $\mathcal{H}$ be the set of all homotopy classes of mappings $\Theta: \Sigma \rightarrow E(M)$ with (2.2). Given $(x, \rho) \in \Sigma \times E(M)$, let $D[x, \rho]$ be the set of all injective real-linear operators $A: T_{x} \Sigma \rightarrow T_{y} M$ such that $y \in M$ is the image of $\rho$ under the bundle projection $E(M) \rightarrow M$ and the image $A\left(T_{x} \Sigma\right)$ is a totally real subspace of $T_{y} M$ satisfying the condition $\mathfrak{L}\left(A\left(T_{x} \Sigma\right)\right)=\rho$ for $\mathfrak{L}$ as in (3.1) with $V=T_{y} M$. The Lie group $G_{x, \rho}$ of all complex automorphisms $B$ of $T_{y} M$ with $\operatorname{det}_{\mathbf{C}} B \in \mathbf{R}$, for $y$ as above, now acts on $D[x, \rho]$ simply transitively by the left multiplication, giving rise to a homotopy equivalence $D[x, \rho] \approx \mathrm{SU}(2) \times \mathbf{Z}_{2}$.

In view of Proposition 3.1 the assignment $f \mapsto \mathfrak{M}(f)$ descends to a mapping $\mathcal{T} \rightarrow \mathcal{H}$, where $\mathcal{T}$ is the set of all $\sim_{\text {tri }}$ equivalence classes of totally real immersions $\Sigma \rightarrow M$. To show that $\mathcal{T} \rightarrow \mathcal{H}$ is surjective, let $\Theta: \Sigma \rightarrow E(M)$ be continuous and satisfy (2.2), and let $\Lambda$ be (the total space of) the bundle over $\Sigma$ with the fibres $\Lambda_{x}=D[x, \Theta(x)]$, for $x \in \Sigma$.

Then $\Lambda$ has two connected components: they are bundles over $\Sigma$, and their fibres, homotopy-equivalent to $\mathrm{SU}(2)$, are connected components of the fibres of $\Lambda$. In fact, as stated at the very end of the last section, we may choose a vector-bundle isomorphism $F$ between $\operatorname{det}_{\mathbf{R}} T \Sigma$ and $\Theta$ treated as a real-line bundle over $\Sigma$. A selection of one connected component of $\Lambda_{x}$, varying continuously with $x \in \Sigma$, consists of those $A \in \Lambda_{x}$ inducing real-line isomorphisms $\left[T_{x} \Sigma\right]^{\wedge 2} \rightarrow \Theta_{x}$ equal to $F_{x}$ times a positive factor.

Using a CW-decomposition of the surface $\Sigma$ we now see that (a connected component of) $\Lambda$ admits a continuous section, and so surjectivity of $\mathcal{T} \rightarrow \mathcal{H}$ follows from the $h$-principle for totally real immersions [20, p. 192].

Finally, to show that $\mathcal{T} \rightarrow \mathcal{H}$ is injective, consider two totally real immersions $f, f^{\prime}: \Sigma \rightarrow M$ with $\mathfrak{M}(f)=\mathfrak{M}\left(f^{\prime}\right)$, and choose a homotopy $[0,1] \ni t \mapsto \Theta_{t}$ between the mappings $\Theta_{0}=\Theta(f)$ and $\Theta_{1}=\Theta\left(f^{\prime}\right)$ defined as in the line following (3.2). If $\Xi$ now is the bundle over $\Sigma \times[0,1]$ with the fibres $D\left[x, \Theta_{t}(x)\right]$ for all $(x, t) \in \Sigma \times[0,1]$, a CW-decomposition argument shows, as before, that (a connected component of) $\Xi$ admits a continuous section which coincides with $d f$ on $\Sigma \times\{0\}$ and with $d f^{\prime}$ on $\Sigma \times\{1\}$. More precisely, if an extension of $d f$ to a section of $\Xi$ thus obtained has a restriction to $\Sigma \times\{1\}$ lying in a different connected component than that containing $d f^{\prime}$, a correction can be made by extending the original homotopy to one parametrized by $t \in[0,2]$, with $\Theta_{t}=e^{2(t-1) \pi i} \Theta_{1}$ for $t \in[1,2]$ (multiplication in the principal $\mathrm{U}(1)$-bundle $E(M)$ ).

Hence, according to the $h$-principle [20, p. 192], $f$ and $f^{\prime}$ are homotopic through totally real immersions, which proves Theorem 2.1.

Note that, as $\mathrm{SU}(3)$ is 2-connected, the surjectivity part of the above argument is still valid when the dimension $n=2$ is replaced by $n=3$. 


\section{TOPOLOGICAL OBSTRUCtions}

All facts presented in this section are well known.

Every almost complex manifold carries a natural orientation. Specifically, an $n$-dimensional complex vector space $V(1 \leq n<\infty)$ becomes an oriented real vector space if one declares the real basis $e_{1}, i e_{1}, \ldots, e_{n}, i e_{n}$ to be positive oriented for some (or any) complex basis $e_{1}, \ldots, e_{n}$. With this convention, the effect on the orientation of the direct sum operation for complex spaces agrees with that for oriented real spaces. For the oriented totally real subspace $W=\operatorname{Span}_{\mathbf{R}}\left\{e_{1}, \ldots, e_{n}\right\}$ of $V$, with the orientation of $i W$ obtained using the direct-sum requirement (that $V=W \oplus i W$ as oriented spaces), the isomorphism $W \rightarrow i W$ of multiplication by $i$ "multiplies" the orientation by the sign factor $(-1)^{n(n-1) / 2}$.

Let $f$ now be a totally real immersion of a real $n$-manifold $\Sigma$ in an almost complex manifold $M$ with $\operatorname{dim}_{\mathbf{C}} M=n$. The multiplication by $i$ provides an isomorphic identification between the tangent bundle $\tau$ of $\Sigma$ (treated as a subbundle of $\left.f^{*} T M\right)$ and the normal bundle $\nu$ of $f$. Thus,

$$
\tau=(-1)^{n(n-1) / 2} \nu, \text { where } \tau=d f(T \Sigma) \text { and } \nu=\left[f^{*} T M\right] / \tau .
$$

The factor $(-1)^{n(n-1) / 2}$ represents the orientation if $\Sigma$ is oriented, and is to be ignored otherwise. In the former case, the isomorphism $\tau \approx \nu$ in (5.1) is orientation-preserving if and only if $n \equiv 0$ or $n \equiv 1 \bmod 4$.

Let $f: \Sigma \rightarrow M$ be a totally real immersion of a $k$-dimensional real manifold $\Sigma$ in an almost complex manifold $M$ with $\operatorname{dim}_{\mathbf{C}} M=n$. Thus, $k \leq n$, and we have an obvious isomorphic identification $\operatorname{Span}_{\mathbf{C}} \tau=[T \Sigma]^{\mathbf{C}}$ of complex vector bundles over $\Sigma$, with $\tau \subset f^{*} T M$ as in (5.1), and [ ] ${ }^{\mathbf{C}}$ denoting complexification. In fact, since $f$ is an immersion, $\tau$ is isomorphic to $T \Sigma$, while $\operatorname{Span}_{\mathbf{C}} \tau=\tau \oplus i \tau \approx \tau^{\mathbf{C}}$ as $f$ is totally real.

If, in addition, $\Sigma$ is closed and $\operatorname{dim}_{\mathbf{R}} \Sigma=\operatorname{dim}_{\mathbf{C}} M$, we can rewrite the relation $\operatorname{Span}_{\mathbf{C}} \tau=[T \Sigma]^{\mathbf{C}}$ as $\operatorname{Span}_{\mathbf{C}}[d f(T \Sigma)]=f^{*} T M$, which, followed by the operation $\operatorname{det}_{\mathbf{C}}$, leads to natural isomorphic identifications:

$$
\text { i) } f^{*} T M=[T \Sigma]^{\mathbf{C}}, \quad \text { ii) } f^{*}\left[\operatorname{det}_{\mathbf{C}} T M\right]=\left[\operatorname{det}_{\mathbf{R}} T \Sigma\right]^{\mathbf{C}} \text {. }
$$

Here and in the sequel, given a real manifold $\Sigma$ with $\operatorname{dim}_{\mathbf{R}} \Sigma=n$ (or, an almost complex manifold $M$ with $\operatorname{dim}_{\mathbf{C}} M=n$ ), we will denote by $\operatorname{det}_{\mathbf{R}} T \Sigma=[T \Sigma]^{\wedge n}$ or $\operatorname{det}_{\mathbf{C}} T M=[T M]^{\wedge n}$ the determinant bundle of the tangent bundle, that is, its highest real/complex exterior power. Taking $w_{2}$ (or $c_{1}$ ) of both sides in (5.2) ii) and noting that $\operatorname{det}_{\mathbf{R}} T \Sigma$ is trivial if $\Sigma$ is orientable, we obtain

$$
\begin{aligned}
& \text { a) } f^{*}\left[w_{2}(M)\right]=w_{1}(\Sigma) \smile w_{1}(\Sigma) \text { in } H^{2}\left(\Sigma, \mathbf{Z}_{2}\right) \\
& \text { b) } f^{*}\left[c_{1}(M)\right]=0 \text { in } H^{2}(\Sigma, \mathbf{Z}) \text { whenever } \Sigma \quad \text { is orientable. }
\end{aligned}
$$

If, in addition, $f: \Sigma \rightarrow M$ is a totally real embedding and the closed manifold $\Sigma$ with $\operatorname{dim}_{\mathbf{R}} \Sigma=\operatorname{dim}_{\mathbf{C}} M=n$ is orientable, we have

$$
f_{*}[\Sigma] \cdot f_{*}[\Sigma]=(-1)^{n(n-1) / 2} \chi(\Sigma),
$$

where $f_{*}[\Sigma] \in H_{n}(M, \mathbf{Z})$ corresponds to either fixed orientation of $\Sigma$ and the dot . denotes the $\mathbf{Z}$-valued intersection form. In fact, the Euler class e $(\nu)$ of the normal bundle $\nu=\nu_{f}$ of any embedding $f: \Sigma \rightarrow M$, integrated over $\Sigma$, yields $f_{*}[\Sigma] \cdot f_{*}[\Sigma]$, while for totally real embeddings $f$, (5.1) gives $\int_{\Sigma} \mathrm{e}(\nu)=$ 
$(-1)^{n(n-1) / 2} \chi(\Sigma)$. When $\Sigma$ is not assumed orientable, instead of (5.4) the same argument gives

$$
f_{*}[\Sigma] \cdot f_{*}[\Sigma]=[\chi(\Sigma) \bmod 2],
$$

where, this time, $f_{*}[\Sigma] \in H_{n}\left(M, \mathbf{Z}_{2}\right)$ and $\cdot$ takes values in $\mathbf{Z}_{2}$.

Formula (5.4) has the following obvious consequences.

Proposition 5.1. Let a closed, orientable manifold $\Sigma$ of even real dimension $n \geq 2$ admit a totally real embedding in $M=\mathbf{C P}^{n}$ or in the complex manifold $M=\mathbf{C P}^{n} \# \overline{\mathbf{C P}^{n}}$ obtained from $\mathbf{C P}^{n}$ by blowing up a point.

(i) If $M=\mathbf{C P}^{n}$, then $(-1)^{n / 2} \chi(\Sigma) \geq 0$ and either $\chi(\Sigma) \equiv 0 \bmod 4$ or $\chi(\Sigma) \equiv 1 \bmod 4$.

(ii) If $M=\mathbf{C P}^{n} \# \overline{\mathbf{C P}^{n}}$, then $\chi(\Sigma)$ is either odd or divisible by 4 .

Proof. The (quadratic) intersection form in $H^{n}(M, \mathbf{Z})$ is algebraically equivalent to $\mathbf{Z} \ni p \mapsto p^{2}$ or $\mathbf{Z} \oplus \mathbf{Z} \ni(p, q) \mapsto p^{2}-q^{2}$. As $p^{2}=p p$ and $p^{2}-q^{2}=(p+q)(p-q)$, with both factors even or both odd, our claim follows from (5.4).

Corollary 5.2. If $n$ is even, $S^{n}$ admits no totally real embedding in $\mathbf{C P}^{n}$ or $\mathrm{CP}^{n} \# \overline{\mathbf{C P}^{n}}$.

Finally, given a continuous mapping $f: \Sigma \rightarrow M$ of a closed real surface $\Sigma$ into an almost complex surface $M$, conditions (5.3) are not only necessary for $f$ to be homotopic to a totally real immersion $\Sigma \rightarrow M$, but also sufficient. This is clear from Theorem 2.1 and Remark 3.2 since $c_{1}$ and $w_{2}$ classify complex line bundles over surfaces [32, p. 798].

\section{Degrees modulo $q$ For CIRCle-VAlued MAPPINGS}

Whenever $\Sigma$ is a manifold, $q \in\{1,2,3, \ldots, \infty\}$, and $g: \Sigma \rightarrow \mathrm{U}(1)$ is a continuous mapping, we define $[g \bmod q] \in H^{1}\left(\Sigma, \mathbf{Z}_{q}\right)=\operatorname{Hom}\left(\pi_{1} \Sigma, \mathbf{Z}_{q}\right)$ to be the composite homomorphism $\pi_{1} \Sigma \rightarrow \mathbf{Z} \rightarrow \mathbf{Z}_{q}$ of the action of $g$ on the fundamental groups with the projection $\mathbf{Z} \rightarrow \mathbf{Z}_{q}$, where $\mathbf{Z}_{1}=\{0\}$ and $\mathbf{Z}_{\infty}=\mathbf{Z}$.

(i) $[g \bmod q]$, as a homomorphism $\pi_{1} \Sigma \rightarrow \mathbf{Z}_{q}$, sends the homotopy class of any loop $S^{1} \rightarrow \Sigma$ to the remainder modulo $q$ of the degree of the composite $S^{1} \rightarrow \Sigma \rightarrow \mathrm{U}(1)$, in which the loop is followed by $g$.

(ii) $[g \bmod q]=0$ if and only if either $q=\infty$ and $g$ has a lift $\Sigma \rightarrow \mathbf{R}$ to the universal covering of $\mathrm{U}(1)$, or $q<\infty$ and the $q$ th root of $g$ treated as a complex-valued function, with $\mathrm{U}(1)=S^{1} \subset \mathbf{C}$, has a single-valued continuous branch $\Sigma \rightarrow \mathrm{U}(1) \subset \mathbf{C}$.

In fact, (i) is obvious and easily implies (ii).

Let $E$ now be any principal $\mathrm{U}(1)$-bundle over a simply connected manifold $M$. As (1.1) is exact, $\pi_{1} E=\mathbf{Z}_{q}$ for some $q \in\{1,2,3, \ldots, \infty\}$, with $\mathbf{Z}_{1}=\{0\}$ and $\mathbf{Z}_{\infty}=\mathbf{Z}$. Given a manifold $\Sigma$ and a continuous mapping $\Theta: \Sigma \rightarrow E$, let us define $\boldsymbol{j}(\Theta) \in H^{1}\left(\Sigma, \mathbf{Z}_{q}\right)$ to be the homomorphism of the fundamental groups induced by $\Theta$ (cf. (1.5) ). Then, for any continuous mapping $g: \Sigma \rightarrow \mathrm{U}(1)$, with $[g \bmod q]$ as above, we have

$$
\boldsymbol{j}(g \Theta)=\boldsymbol{j}(\Theta)+[g \bmod q],
$$

$g \Theta$ being the valuewise product. (This is clear from (i) in Section 6 , since a principal $\mathrm{U}(1)$-bundle over $S^{1}$ is trivial.) 


\section{Proof of Theorem 2.2. Surjectivity}

In view of Theorem 2.1, the assertion of Theorem 2.2 amounts to bijectivity of the assignment given, in the notation of Section 6 and (2.4), by

$$
[\Theta] \mapsto(\boldsymbol{i}, \boldsymbol{d}) \quad \text { with } \boldsymbol{i}=\boldsymbol{j}(\Theta) \text { and } \boldsymbol{d}=\boldsymbol{d}(\pi \circ \Theta) .
$$

Explicitly, (7.1) sends the homotopy class of any mapping $\Theta: \Sigma \rightarrow E(M)$ with (2.2) to the pair $(\boldsymbol{i}, \boldsymbol{d})$ formed by $\boldsymbol{i} \in H^{1}\left(\Sigma, \mathbf{Z}_{q}\right)=\operatorname{Hom}\left(\pi_{1} \Sigma, \mathbf{Z}_{q}\right)$ which is the action of $\Theta$ on the fundamental groups, and $\boldsymbol{d}$ which is the image of the fundamental homology class $[\Sigma] \in H_{2}\left(\Sigma, \mathbf{Z}_{[2]}\right)$ under $\pi \circ \Theta$. (Here $\mathbf{Z}_{[2]}$ is defined as in (2.3), and $\pi: E(M) \rightarrow M$ stands for the bundle projection.) Recall that $\boldsymbol{i}$ and $\boldsymbol{d}$ in (7.1) are the Maslov index and degree of a totally real immersion $f$ of $\Sigma$ in $M$ whose $\sim_{\text {tri }}$ equivalence class corresponds to $[\Theta]$ as in Theorem 2.1 since $\pi \circ \Theta$ then is homotopic to $f$.

In this section we prove the surjectivity part of Theorem 2.2, Injectivity will be established in Section 8. We begin with a lemma:

Lemma 7.1. Given a simply connected almost complex surface $M$ and a closed real surface $\Sigma$, let $q, \mathfrak{I}_{q}(\Sigma), \mathfrak{D}_{ \pm}^{\varepsilon}(M), \varepsilon$ and \pm be defined by (1.4) and (2.7)-(2.9).

(a) $(\boldsymbol{i}, \boldsymbol{d}) \in \mathfrak{I}_{q}(\Sigma) \times \mathfrak{D}_{ \pm}^{\varepsilon}(M)$ if $(\boldsymbol{i}, \boldsymbol{d})$ corresponds as in (7.1) to a continuous mapping $\Theta: \Sigma \rightarrow E(M)$ satisfying (2.2).

(b) $\mathbf{i}(f) \in \mathfrak{I}_{q}(\Sigma) \subset H^{1}\left(\Sigma, \mathbf{Z}_{q}\right)$ and $\boldsymbol{d}(f) \in \mathfrak{D}_{ \pm}^{\varepsilon}(M) \subset H_{2}\left(M, \mathbf{Z}_{[2]}\right)$ whenever $f: \Sigma \rightarrow M$ is a totally real immersion and $\mathbf{i}(f), \boldsymbol{d}(f)$ are its Maslov index and degree.

Proof. As $\pi_{1}\left[E^{+}(M)\right]=2 \mathbf{Z}_{q} \subset \mathbf{Z}_{q}$ by (1.3)-(1.4), $\mathfrak{w}_{1}$ in (2.1) must be the unique nonzero homomorphism $\mathbf{Z}_{q} \rightarrow \mathbf{Z}_{2}$. For $\Theta$ as in (a), relation (2.2) states that $\mathfrak{w}_{1} \circ \Theta_{*}=w_{1}(\Sigma)$, and so $w_{1}(\Sigma)$ is the $\bmod 2$ reduction of $\boldsymbol{i}$ (see Remark 1.1). Hence $\boldsymbol{i} \in \mathfrak{I}_{q}(\Sigma)$. Next, $\boldsymbol{d}(f) \in \mathfrak{D}_{ \pm}^{\varepsilon}(M)$ in view of Remark 3.2 (ii) for $f=\pi \circ \Theta$ and (1.7), which proves (a). Finally, (b) follows from (a) and Proposition 3.1. completing the proof.

Let $\mathcal{Z}$ be the image of the mapping (7.1). Thus, $\mathcal{Z} \subset \mathfrak{I}_{q}(\Sigma) \times \mathfrak{D}_{ \pm}^{\varepsilon}(M)$ by Lemma 7.1(a). To prove surjectivity in Theorem 2.2. we show that $\mathcal{Z}$ is the set $\mathcal{Z}(\Sigma, M)$ defined immediately after Theorem 2.2. First, if $\mathfrak{D}_{ \pm}^{\varepsilon}(M)=\varnothing$, our claim follows as $\mathcal{Z}=\mathfrak{I}_{q}(\Sigma) \times \mathfrak{D}_{ \pm}^{\varepsilon}(M)=\varnothing$.

From now on, we may thus assume that $\mathfrak{D}_{ \pm}^{\varepsilon}(M) \neq \varnothing$. Let us now fix any $\boldsymbol{d} \in \mathfrak{D}_{ \pm}^{\varepsilon}(M)$ and describe the set of all $\boldsymbol{i} \in \mathfrak{I}_{q}(\Sigma)$ with $(\boldsymbol{i}, \boldsymbol{d}) \in \mathcal{Z}$.

Since $M$ is simply connected, $\boldsymbol{d}$ (or, in fact, any class in $H_{2}\left(M, \mathbf{Z}_{[2]}\right)$ ) is realized by a mapping $S^{2} \rightarrow M$ and, with the aid of a degree 1 map $\Sigma \rightarrow S^{2}$, also by a mapping $f: \Sigma \rightarrow M$. However, $\boldsymbol{d}=\boldsymbol{d}(f) \in \mathfrak{D}_{ \pm}^{\varepsilon}(M)$, and so relations (2.8), (2.7) and (1.7) show that either $\Sigma$ is orientable and $f^{*}\left[\operatorname{det}_{\mathbf{C}} T M\right]$ is trivial, or $\Sigma$ is nonorientable and the line bundles $f^{*}\left[\operatorname{det}_{\mathbf{C}} T M\right]$ and $\left[\operatorname{det}_{\mathbf{R}} T \Sigma\right]^{\mathbf{C}}$ have the same $w_{2}$. Thus, in either case, $f^{*}\left[\operatorname{det}_{\mathbf{C}} T M\right]$ and $\left[\operatorname{det}_{\mathbf{R}} T \Sigma\right]^{\mathbf{C}}$ are isomorphic [32, p. 798]; hence, by Remark 3.2(ii), $f$ admits a continuous lift $\Theta: \Sigma \rightarrow E(M)$ with (2.2).

Once such $\Theta$ is fixed, those $\boldsymbol{i} \in \mathfrak{I}_{q}(\Sigma)$ for which $(\boldsymbol{i}, \boldsymbol{d}) \in \mathcal{Z}$ are precisely the values $\boldsymbol{j}(g \Theta)$ for all continuous mappings $g: \Sigma \rightarrow \mathrm{U}(1)$ (notation of Section 6) with the property that the homomorphism $[g \bmod q]: \pi_{1} \Sigma \rightarrow \mathbf{Z}_{q}$ defined in Section 6 is valued in the even subgroup $2 \mathbf{Z}_{q}$ of $\mathbf{Z}_{q}$. In fact, $\boldsymbol{d}$ determines the homotopy class of $f: \Sigma \rightarrow M$ uniquely [35], which, combined with an obvious homotopy-lifting argument, shows that the elements of $\mathcal{Z}$ having the form $(\boldsymbol{i}, \boldsymbol{d})$ 
are images under (7.1) of products $g \Theta$. However, since $\Theta$ satisfies (2.2), condition (2.2) for $g \Theta$ (rather than $\Theta$ ) is equivalent to even-valuedness of $[g \bmod q]$, as $\Theta^{*}$ in (2.2) is dual to $\boldsymbol{j}(\Theta)$.

The set $\left\{\boldsymbol{i} \in \mathfrak{I}_{q}(\Sigma):(\boldsymbol{i}, \boldsymbol{d}) \in \mathcal{Z}\right\}$ is thus nonempty (as it contains $\boldsymbol{j}(\Theta)$ ), and hence, by (6.1), it is a coset, in $\operatorname{Hom}\left(\pi_{1} \Sigma, 2 \mathbf{Z}_{q}\right)$, of the subgroup $\mathcal{G}$ consisting of those elements of $\operatorname{Hom}\left(\pi_{1} \Sigma, 2 \mathbf{Z}_{q}\right)$ which have the form $[g \bmod q]$, with a continuous mapping $g: \Sigma \rightarrow \mathrm{U}(1)$. Therefore $\mathfrak{I}_{q}(\Sigma) \neq \varnothing$, and, by (2.9) and Remark 1.1. $\mathfrak{I}_{q}(\Sigma)$ is a coset of $\operatorname{Hom}\left(\pi_{1} \Sigma, 2 \mathbf{Z}_{q}\right)$ in $\operatorname{Hom}\left(\pi_{1} \Sigma, \mathbf{Z}_{q}\right)=H^{1}\left(\Sigma, \mathbf{Z}_{q}\right)$.

First, suppose that $\Sigma$ is orientable, or $q=\infty$, or $\chi(\Sigma)$ is odd, or $q$ is finite but not divisible by 4 . In these four cases, $\mathcal{G}=\operatorname{Hom}\left(\pi_{1} \Sigma, 2 \mathbf{Z}_{q}\right)$ (and hence $\mathcal{Z}=$ $\mathfrak{I}_{q}(\Sigma) \times \mathfrak{D}_{ \pm}^{\varepsilon}(M)$ according to the last paragraph). In fact, for every closed surface $\Sigma$, continuous mappings $g: \Sigma \rightarrow \mathrm{U}(1)$ realize all homomorphisms $\pi_{1} \Sigma \rightarrow \mathbf{Z}=$ $\pi_{1}[\mathrm{U}(1)]$, while, in each of the four cases, a homomorphism $H_{1}(\Sigma, \mathbf{Z}) \rightarrow \mathbf{Z}_{q}$ valued in $2 \mathbf{Z}_{q}$ is necessarily the composite $H_{1}(\Sigma, \mathbf{Z}) \rightarrow \mathbf{Z} \rightarrow \mathbf{Z}_{q}$ of some homomorphism $H_{1}(\Sigma, \mathbf{Z}) \rightarrow \mathbf{Z}$ and the projection $\mathbf{Z} \rightarrow \mathbf{Z}_{q}$. In the first two cases, this is obvious since $H_{1}(\Sigma, \mathbf{Z})$ is free, or, respectively, $\mathbf{Z}_{\infty}=\mathbf{Z}$. In the last two cases, $\Sigma$ may thus be assumed nonorientable, and $q$ finite. Then $H_{1}(\Sigma, \mathbf{Z})$ has just one nontrivial element $\xi$ of finite order, namely, of order 2. (See Remark 1.2.) Case three now implies case four: if $\chi(\Sigma)$ is odd, $\xi \notin \operatorname{Ker}\left[w_{1}(\Sigma)\right]$, and so the image of $\xi$ under any homomorphism that lies in $\mathfrak{I}_{q}(\Sigma)$ necessarily equals $q / 2$, due to its being odd in $\mathbf{Z}_{q}$ and of order two; hence $q / 2$ is odd as $\mathfrak{I}_{q}(\Sigma) \neq \varnothing$. Next, in the fourth case, any homomorphism $H_{1}(\Sigma, \mathbf{Z}) \rightarrow 2 \mathbf{Z}_{q}$ sends $\xi$ to 0 , since $q / 2$, the only nontrivial element of order 2 in $\mathbf{Z}_{q}$, is odd.

Finally, let us assume that $\Sigma$ is nonorientable, $\chi(\Sigma)$ is even, and $q$ is a finite multiple of 4. A homomorphism $\varphi: H_{1}(\Sigma, \mathbf{Z}) \rightarrow 2 \mathbf{Z}_{q}$ sends the torsion element $\xi \in H_{1}(\Sigma, \mathbf{Z})$ either to 0 or to $q / 2$, and only those homomorphisms $\varphi$ with $\varphi(\xi)=0$ have factorizations $H_{1}(\Sigma, \mathbf{Z}) \rightarrow \mathbf{Z} \rightarrow \mathbf{Z}_{q}$ as above, that is, lie in $\mathcal{G}$. Hence $\mathcal{G}$ is an index 2 subgroup of $\operatorname{Hom}\left(\pi_{1} \Sigma, 2 \mathbf{Z}_{q}\right)$, which shows that, in this case, $\mathcal{Z}$ has half the number of elements of $\mathfrak{I}_{q}(\Sigma) \times \mathfrak{D}_{ \pm}^{\varepsilon}(M)$. The relation $\mathcal{Z}=\mathcal{Z}(\Sigma, M)$ will now follow once we show that the image of $\xi$ under some (or any) $\boldsymbol{i}$ with $(\boldsymbol{i}, \boldsymbol{d}) \in \mathcal{Z}$ equals 0 if and only if our $\boldsymbol{d}$ is the $\bmod 2$ reduction of an element of $\operatorname{Ker}\left[c_{1}(M)\right] \subset H_{2}(M, \mathbf{Z})$.

To establish the 'only if' part of this last statement, let some such $\boldsymbol{i}$ send $\xi$ to 0 . For a suitably chosen embedded circle $\Gamma \subset \Sigma$ representing $\xi$ in homology, $\Sigma \backslash \Gamma$ is the interior of a compact orientable surface with a boundary formed by two circles. (Cf. case (b) of Remark 25.1 in Section 25) Capping the two circles with two copies of a 2-disk $D$, we obtain a closed orientable surface $\Sigma^{\prime}$ such that $\Sigma$ is homeomorphic to $\Sigma^{\prime} \# K^{2}$, where $K^{2}$ is the Klein bottle. A mapping $\Theta: \Sigma \rightarrow E(M)$ with (2.2) that realizes $(\boldsymbol{i}, \boldsymbol{d})$ as in (7.1) now gives rise to a mapping $\Theta^{\prime}: \Sigma^{\prime} \rightarrow E(M)$ equal to $\Theta$ on $\Sigma \backslash \Gamma$ and obtained on both copies of $D$ by extending $\Theta$ from $\Gamma$ to $D$, which is possible as $\boldsymbol{i}$ sends $\xi$ to 0 . In addition, $\Theta^{\prime}$ still satisfies (2.2): every element of $H_{1}\left(\Sigma^{\prime}, \mathbf{Z}\right)$ is represented by a loop $\gamma: S^{1} \rightarrow \Sigma \backslash \Gamma$, for which $\gamma^{*}(T \Sigma)$ is orientable (since so is $\gamma^{*}\left(T \Sigma^{\prime}\right)$ ), and, therefore, $\Theta^{\prime} \circ \gamma=\Theta \circ \gamma$ can be lifted to a loop in $E^{+}(M)$. Thus, by Lemma 7.1(a), $\boldsymbol{d}^{\prime}=\boldsymbol{d}(\pi \circ \Theta)$ lies in $\mathfrak{D}_{+}^{1}(M)=\operatorname{Ker}\left[c_{1}(M)\right]$, and the $\bmod 2$ reduction of $\boldsymbol{d}^{\prime}$ is $\boldsymbol{d}$, due to mutual cancellation of the contibutions from the two copies of $D$.

For the 'if' part, let $\boldsymbol{d}$ be the $\bmod 2$ reduction of $\boldsymbol{d}^{\prime} \in \operatorname{Ker}\left[c_{1}(M)\right]$, and let $\Sigma^{\prime}$ be a closed orientable real surface with $\Sigma^{\prime} \# K^{2}=\Sigma$. For reasons given in 
the third paragraph after the proof of Lemma 7.1 (but now applied to $\boldsymbol{d}^{\prime}, \Sigma^{\prime}$ and $\mathfrak{D}_{+}^{1}(M)=\operatorname{Ker}\left[c_{1}(M)\right]$ rather than $\boldsymbol{d}, \Sigma$ and $\left.\mathfrak{D}_{ \pm}^{\varepsilon}(M)\right)$, one can realize $\boldsymbol{d}^{\prime}$ by a continuous mapping $f^{\prime}: \Sigma^{\prime} \rightarrow M$, and any such $f^{\prime}$ admits a continuous lift $\Theta^{\prime}: \Sigma^{\prime} \rightarrow E(M)$ satisfying (2.2). Next, let a mapping $\Theta^{\prime \prime}: K^{2} \rightarrow E(M)$ from the Klein bottle into $E(M)$ be obtained as the composite of the bundle projection $K^{2} \rightarrow S^{1}$ followed by a homeomorphism of $S^{1}$ onto a fibre, intersecting $\Theta^{\prime}\left(\Sigma^{\prime}\right)$, of the principal $\mathrm{U}(1)$-bundle $E(M)$. Without changing the homotopy class of $\Theta^{\prime}$ or $\Theta^{\prime \prime}$, we may further assume that they map some small nonempty open sets $U^{\prime} \subset \Sigma^{\prime}$ and $U^{\prime \prime} \subset K^{2}$ onto a single point of $E(M)$. Modifying both mappings in closed 2-disks $D^{\prime} \subset U^{\prime}$ and $D^{\prime \prime} \subset U^{\prime \prime}$, we obtain a mapping $\Theta: \Sigma=\Sigma^{\prime} \# K^{2} \rightarrow E(M)$ that is constant on the tube connecting $\Sigma^{\prime} \backslash D^{\prime}$ to $K^{2} \backslash D^{\prime \prime}$ in $\Sigma^{\prime} \# K^{2}$, while $\Theta=\Theta^{\prime}$ on $\Sigma^{\prime} \backslash D^{\prime}$ and $\Theta=\Theta^{\prime \prime}$ on $K^{2} \backslash D^{\prime \prime}$. It is now clear that $\Theta$ sends $\xi$ to 0 and satisfies (2.2): $\xi$ is represented by a fibre of the bundle projection $K^{2} \rightarrow S^{1}$, on which $\Theta$ is constant, while $\xi$ and the homology classes of loops lying in $\Sigma^{\prime} \backslash D^{\prime}$ generate the kernel of $w_{1}(\Sigma): H_{1}(\Sigma, \mathbf{Z}) \rightarrow \mathbf{Z}_{2}$, so that the $\Theta$-images of these loops can be lifted to loops in $E^{+}(M)$ due to orientability of $\Sigma^{\prime}$. However, no such lifts exist for a circle in $K^{2}$ forming a section of the bundle $K^{2} \rightarrow S^{1}$, as its $\Theta$-image is a fibre of $E(M)$ (and so any lift is a semicircle in a fibre of $E^{+}(M)$ ).

\section{Proof of Theorem 2.2, Injectivity}

To prove injectivity of (7.1), let two mappings $\Theta, \Theta^{\prime}: \Sigma \rightarrow E(M)$, both satisfying (2.2), have $\boldsymbol{j}(\Theta)=\boldsymbol{j}\left(\Theta^{\prime}\right)=\boldsymbol{i}$ and $\boldsymbol{d}(\pi \circ \Theta)=\boldsymbol{d}\left(\pi \circ \Theta^{\prime}\right)=\boldsymbol{d}$. As $\boldsymbol{d}(f)=f_{*}[\Sigma] \in H_{2}\left(M, \mathbf{Z}_{[2]}\right)$ uniquely determines the homotopy class of $f: \Sigma \rightarrow M$ (see [35]), we can lift a fixed homotopy between $f=\pi \circ \Theta$ and $f^{\prime}=\pi \circ \Theta^{\prime}$ to the principal $\mathrm{U}(1)$-bundle $E(M)$ over $M$, obtaining a homotopy between $\Theta^{\prime}$ and some lift $g \Theta$ of $f=\pi \circ \Theta$ to $E(M)$, where $g: \Sigma \rightarrow \mathrm{U}(1)$ is a suitable continuous mapping. By (6.1), $[g \bmod q]=0$. It now suffices to show that $\Theta$ and $g \Theta$ are homotopic. We may assume that $q<\infty$, for otherwise $g$ is homotopic to a constant mapping ((ii) in Section 6).

The required homotopy $\Xi: \Sigma \times[0,1] \rightarrow E(M)$ with $\Xi(\cdot, 0)=\Theta$ and $\Xi(\cdot, 1)=$ $g \Theta$ will be built on successive skeleta of a specific CW-decomposition of $\Sigma$, namely, one resulting from a surjective continuous mapping $\phi: D \rightarrow \Sigma$, where $D$ is an oriented 2-disk with the accordingly oriented boundary circle $\partial D$. We choose $\phi$ so that $\partial D$ is partitioned by some $2 k$-element subset, $k \geq 1$, into $2 k$ compact boundary segments, and the only identifications of points of $D$ under $\phi$ are those provided by some $k$ homeomorphisms between pairs of boundary segments. Replacing $\Xi$ by $\hat{\Xi}: D \times[0,1] \rightarrow E(M)$ with $\hat{\Xi}(z, t)=\Xi(\phi(z), t)$ for all $(z, t) \in D \times[0,1]$, we see that, at any stage, instead of $\Xi$ we may just construct $\hat{\Xi}$, which depends on $(z, t)$ only through $(\phi(z), t)$.

First, we choose a single-valued continuous function $h: \Sigma \rightarrow \mathrm{U}(1) \subset \mathbf{C}$ with $h^{q}=g$. (See (ii) in Section [6.) Now, for any point $x$ in the 0-skeleton of $\Sigma$ (the $\phi$-image of the $2 k$-element partitioning set), we let $t_{j}=j / q$ and $\Xi(x, t)=$ $[h(x)]^{j} \gamma_{x}\left(t-t_{j}\right) \Theta(x)$ for $t \in\left[t_{j}, t_{j+1}\right]$ and $j \in\{0, \ldots, q-1\}$, with any fixed curve $\gamma_{x}:[0,1 / q] \rightarrow \mathrm{U}(1)$ joining 1 to $h(x)$.

An extension of $\hat{\Xi}$ from the 0 -skeleton to the 1 -skeleton is in turn obtained separately on each rectangle $R=S \times[0,1]$, where $S \subset \partial D$ is one of the $2 k$ boundary segments. Our $\hat{\Xi}$ is already defined on the (oriented) boundary $\partial R$ of $R$, so that $\hat{\Xi}: \partial R \rightarrow E(M)$ represents a free homotopy class $\alpha$ of loops in $E(M)$. 
As $\pi_{1}[E(M)]=\mathbf{Z}_{q}$ is Abelian, such free homotopy classes can be meaningfully multiplied and form a group isomorphic to $\pi_{1}[E(M)]$. We now show that $\alpha=\beta^{q}$ for the free homotopy class $\beta$ of some loop $\Pi$, and so $\alpha$ is trivial, which provides an extension of $\hat{\Xi}$ from $\partial R$ to $R$, thus concluding the 1-skeleton step. Specifically, $\Pi: \partial R^{\prime} \rightarrow E(M)$ is defined on the boundary of $R^{\prime}=S \times[0,1 / q]$ by $\Phi(\cdot, 1 / q)=$ $(h \Theta) \circ \phi$ on $S \times\{1 / q\}$ and $\Pi=\hat{\Xi}$ everywhere else. Also, $\alpha=\beta_{0} \ldots \beta_{q-1}$, with $\beta_{j}$ denoting the free homotopy class of the loop $\Pi_{j}: \partial R^{\prime} \rightarrow E(M)$ given by $\Pi_{j}(\cdot, t)=\left(h^{j} \circ \phi\right) \Pi(\cdot, t)$, as one sees noting that for each $j \in\{0, \ldots, q-2\}$ the contribution to $\beta_{j}$ from $S \times\{1 / q\}$ cancels the contribution to $\beta_{j+1}$ from $S \times\{0\}$. On the other hand, $\beta_{j}=\beta$ for all $j$, since the segment $S$ is contractible, and so we may choose a homotopy between the constant mapping 1 and $h: S \rightarrow \mathrm{U}(1)$, which leads to a free homotopy between $\Pi_{j}$ and $\Pi_{j+1}, j=0, \ldots, q-2$.

Now that $\hat{\Xi}$ is already defined on the boundary $\partial C$ of the solid cylinder $C=$ $D \times[0,1]$, our 2 -skeleton step amounts to extending it from $\partial C$ to $C$. Let us denote by $\sigma \in \pi_{2}[E(M)]$ and $\xi \in H_{2}(E(M), \mathbf{Z})$ the homotopy class with any fixed base point, and, respectively, the homology class, of $\hat{\Xi}: \partial C \rightarrow E(M)$ (for the standard orientation of the 2 -sphere $\partial C$ ). There are two cases.

If $\Sigma$ is orientable, $\sigma=0$. In fact, $\xi$ then equals the difference of the homology classes of $\Theta$ and $g \Theta$, for a suitable orientation of $\Sigma$. (Contributions from $\hat{\Xi}$ restricted to $\partial D \times[0,1]$ undergo pairwise cancellations, as all identifications, under $\phi$, of pairs of boundary segments in $\partial D$ are orientation-reversing.) Since $\pi \circ \Theta=$ $\pi \circ(g \Theta)$, we thus get $\pi_{*} \xi=0$ in $H_{2}(M, \mathbf{Z})$, so that, in view of the Hurewicz isomorphism, $\pi_{*} \sigma=0$ in $\pi_{2} M$. Injectivity of the first homomorphism in (1.1) now gives $\sigma=0$, which provides the required extension of $\hat{\Xi}$ from the 2 -sphere $\partial C$ to the 3-disk $C$.

If $\Sigma$ is not orientable, $\sigma$ may depend on how one chose the extension on $\Xi$ from the 0 -skeleton to the 1 -skeleton of $\Sigma$. Namely, if we choose that extension differently, we can modify the resulting $\sigma$ so as to add to it any prescribed even element of $\pi_{2}[E(M)]$, 'even' meaning divisible by 2 in $\pi_{2}[E(M)]$. In fact, at least one identification under $\phi$ of a pair $S, S^{\prime}$ of boundary segments in $\partial D$ is now orientation-preserving. Let us fix such $S, S^{\prime} \subset \partial D$, with $\phi(S)=\phi\left(S^{\prime}\right)$. Any given element $\rho$ of $\pi_{2}[E(M)]$ can be represented by a mapping $F$ from the 2-sphere obtained when two separate copies of the rectangle $R=S \times[0,1]$ are glued together by identifying their boundaries $\partial R$, and $F$ may be chosen so that the restriction of $F$ to one copy of $R$ is the extension of $\hat{\Xi}$ from $\partial R$ to $R$ used in the 1-skeleton step. If we now replace that extension by a new one, namely, by the restriction of $F$ to the other copy of $R$, the corresponding element $\sigma$ of $\pi_{2}[E(M)]$ will be replaced by $\sigma+2 \rho$. (As the identification of $S$ with $S^{\prime}$ under $\phi$ is orientationpreserving, the restriction of either version of $\hat{\Xi}$ to $S \times[0,1]$ contributes twice to the homotopy or homology class.)

Therefore, to show that $\sigma=0$ for a suitably chosen extension to the 1-skeleton, we just need to verify that the original $\sigma$ is an even element of $\pi_{2}[E(M)]$. To this end, first note that $\pi_{*} \xi$ is even in $H_{2}(M, \mathbf{Z})$, since for some pairs $S, S^{\prime}$ of boundary segments identified under $\phi$ the contributions of $\hat{\Xi}: S \times[0,1] \rightarrow E(M)$ to homology count twice (namely, the pairs whose identification is orientation-preserving), while for the remaining pairs the contributions cancel each other, which is also the case for the contributions of $\pi \circ \hat{\Xi}: D \times\{t\} \rightarrow M$ for $t=0$ and $t=1$ (due to their having the same image, with opposite orientations). The Hurewicz 
isomorphism theorem now implies that $\pi_{*} \sigma$ is even in $\pi_{2} M$. If $\sigma$ itself were not even in $\pi_{2}[E(M)]$, an element of $\pi_{2} M$ whose double is $\pi_{*} \sigma$ would project onto a nontrivial element of order 2 in the quotient of $\pi_{2} M$ over the isomorphic image of $\pi_{2}[E(M)]$ under $\pi_{*}$ (cf. (1.1)). This would contradict the fact that, in view of exactness of (1.1), the quotient group in question is isomorphic to a subgroup of $\pi_{1}[\mathrm{U}(1)]=\mathbf{Z}$, and hence torsion-free. Thus, (7.1) is injective, which proves Theorem 2.2 .

\section{The SIMPLest EXAMPLES}

Here begins the second part of the paper, devoted to constructing explicit examples of totally real immersions and embeddings. The simplest such constructions are described in this section. All of them are well known.

Example 9.1. A real subspace $W$ of a complex vector space $V$ with $\operatorname{dim} V<\infty$ is totally real if and only if $\operatorname{Span}_{\mathbf{C}} W$ in $V$ has the complex dimension $\operatorname{dim}_{\mathbf{R}} W$. This means that some (or every) $\mathbf{R}$-basis of $W$ is also linearly independent over $\mathbf{C}$ in $V$. Thus, given complex-valued $C^{1}$ functions $f_{1}, \ldots, f_{n}$ on a nonempty open set $U$ in $\mathbf{R}^{n}$, the mapping $f=\left(f_{1}, \ldots, f_{n}\right)$ is a totally real immersion $U \rightarrow \mathbf{C}^{n}$ if and only if $\mathcal{J}\left(f_{1}, \ldots, f_{n}\right) \neq 0$ at every $x \in U$, where $\mathcal{J}\left(f_{1}, \ldots, f_{n}\right)=\operatorname{det} \mathfrak{F}$ for the complex $n \times n$ Jacobian matrix $\mathfrak{F}=F(x)$ with the entries $\partial f_{j} / \partial x_{k}$.

Example 9.2. Obviously, an embedding $f$ of a real manifold $\Sigma$ in an almost complex manifold $M$ is totally real if and only if so is the image $f(\Sigma)$ as a submanifold of $M$.

Example 9.3. Let $\Sigma=\{(z, v) \in U \times V: v=\varphi(z)\}$ be the graph of a $C^{\infty}$ mapping $\varphi: U \rightarrow V$ from a nonempty connected open set $U \subset \mathbf{C}$ into a complex vector space $V$ with $\operatorname{dim} V<\infty$. Then $\Sigma$ is a totally real submanifold of $U \times V$ if and only if $\varphi$ satisfies, at each point of $U$, the Cauchy-Riemann inequality $\varphi_{\bar{z}} \neq 0$, where $\varphi_{\bar{z}}=\left(\varphi_{x}+i \varphi_{y}\right) / 2$ with $x=\operatorname{Re} z, y=\operatorname{Im} z$, and the subscripts stand for the partial derivatives.

In fact, $\Sigma$ is totally real in $U \times V$ if and only if the graph embedding $U \rightarrow U \times V$ given by $z \mapsto(z, \varphi(z))$ is totally real (Example 9.2), and our claim follows in view of Example 9.1 .

Example 9.4. Given a complex vector space $V$ with $\operatorname{dim}_{\mathbf{C}} V=2$, any real subspace $W \subset V$ with $\operatorname{dim}_{\mathbf{R}} W=2$ which is not totally real must, obviously, be a complex 1-dimensional subspace of $V$. Suppose now that $\Sigma$ is a submanifold of an almost complex manifold $M$ and $\operatorname{dim}_{\mathbf{R}} \Sigma=2$. Removing from $\Sigma$ all complex points, that is, those $x \in \Sigma$ for which $T_{x} \Sigma$ is a complex line in $T_{x} M$, we obtain an open subset $U$ of $\Sigma$ and, if $U$ is nonempty, its connected components are totally real submanifolds of $M$.

Totally real submanifolds naturally arise in many other common situations. For instance, a real submanifold $\Sigma \subset M$ of an almost complex manifold $M$ is totally real in each of the following obvious cases:

(i) $\operatorname{dim} \Sigma=1$.

(ii) $\Sigma$ is a connected component of the fixed-point set $\{x \in M: \bar{x}=x\}$ of any $C^{\infty}$ involution $M \ni x \mapsto \bar{x} \in M$ reversing the almost complex structure. One then has $\operatorname{dim}_{\mathbf{R}} \Sigma=\operatorname{dim}_{\mathbf{C}} M$. 
(iii) $N$ is an almost complex manifold admitting an involution $x \mapsto \bar{x}$ that reverses the almost complex structure and $M$ is the product almost complex manifold $N \times N$, while $\Sigma \subset M$ is the anti-diagonal submanifold $\{(\bar{x}, x): x \in N\}$, diffeomorphic to $N$. (This is clear from (ii) applied to the involution $(x, y) \mapsto(\bar{y}, \bar{x})$ of $N \times N$.)

(iv) $\Sigma=\Sigma^{\prime} \times \Sigma^{\prime \prime}$, where $\Sigma^{\prime} \subset M^{\prime}$ and $\Sigma^{\prime \prime} \subset M^{\prime \prime}$ are totally real submanifolds, and $M=M^{\prime} \times M^{\prime \prime}$ is the product almost complex manifold. (For a more general construction, see Lemma 29.4.)

(v) By (i) and (iv), embedded closed curves $K, K^{\prime} \subset \mathbf{C}$ give rise to a Cliffordlike totally real embedded 2-torus $\Sigma=K \times K^{\prime} \subset M=\mathbf{C}^{2}$. Iterating this produces totally real embedded $n$-tori in $\mathbf{C}^{n}$.

(vi) The standard real form $\Sigma=\mathbf{R P}^{n} \subset M=\mathbf{C P}^{n}$ is a totally real embedded submanifold, which follows from (ii) applied to the involution of $\mathbf{C P}^{n}$ given by $\left[x_{0}, \ldots, x_{n}\right] \mapsto\left[\bar{x}_{0}, \ldots, \bar{x}_{n}\right]$ in projective coordinates.

(vii) Denoting by $x \mapsto \bar{x}$ any antiholomorphic involution of $S^{2}=\mathrm{CP}^{1}$ (e.g., the complex conjugation in $\mathbf{C}$ extended to the Riemann sphere), we see that, by (iii), the anti-diagonal 2 -sphere $\Sigma=\left\{(\bar{x}, x): x \in S^{2}\right\}$ is totally real in $M=S^{2} \times S^{2}=\mathrm{CP}^{1} \times \mathbf{C P}^{1}$.

\section{ZOOMING}

The following well-known fact might be called a zooming principle.

Proposition 10.1. If a compact manifold $\Sigma$ admits a totally real immersion/embedding $f$ in $\mathbf{C}^{n}$, then it admits a totally real immersion/embedding $h$ in every almost complex manifold $M$ of complex dimension $n$. We may choose $h$ to be the composite of $f$ with a suitable $C^{\infty}$-diffeomorphic embedding in $M$ of an open ball in $\mathbf{C}^{n}$ containing $f(\Sigma)$.

Corollary 10.2. For every integer $n \geq 1$, the $n$-torus $T^{n}$ admits a totally real embedding in every almost complex manifold of complex dimension $n$. Such an embedding may be chosen so that its image lies in any prescribed open subset diffeomorphic to a ball.

Proposition 10.1 trivially follows from Lemma 10.4 below (as explained in Remark 10.5), and Corollary 10.2 then is immediate from (v) in Section 9 . First, we need a definition and another lemma.

Given an immersion $\Phi: \Sigma \rightarrow V$ of a real $n$-dimensional manifold $\Sigma$ in a real or complex vector space $V$, the Gauss mapping

$$
\mathrm{G}_{\Phi}: \Sigma \rightarrow \mathrm{Gr}_{n}(V)
$$

of $\Phi$ assigns to each $x \in \Sigma$ the image $d \Phi_{x}\left(T_{x} \Sigma\right)$. Here $\operatorname{Gr}_{n}(V)$ is the Grassmann manifold of all $n$-dimensional real vector subspaces of $V$.

Lemma 10.3. Let $J$ be a complex structure in a real vector space $V$, that is, a linear operator $V \rightarrow V$ with $J^{2}=-\mathrm{Id}$, and let $Y$ be some given set of $J$-totally real subspaces of a fixed dimension $n \geq 0$. If $\operatorname{dim} V<\infty$ and $Y$ is compact as a subset of $\operatorname{Gr}_{n}(V)$, then all $W \in Y$ are totally real relative to every complex structure that lies in a suitable neighborhood $\Omega$ of $J$ in $\operatorname{Hom}_{\mathbf{R}}(V, V)$.

In fact, otherwise there would exist a sequence $J_{k} \in \operatorname{Hom}_{\mathbf{R}}(V, V)$ of complex structures and sequences $W_{k} \in Y$ and $u_{k} \in V$ such that $J_{k} \rightarrow J$ as $k \rightarrow \infty$, 
while $u_{k} \in W_{k} \cap J_{k} W_{k}$ and $\left|u_{k}\right|=1$ for some fixed Euclidean norm || in $V$. Using compactness of $Y$ and the unit sphere, we could pass to subsequences for which $W_{k} \rightarrow W$ and $u_{k} \rightarrow u$ with some $W \in Y$ and $u \in V$, so that $u \in W \cap J W$ and $|u|=1$, contradicting the assumption that $W \cap J W=\{0\}$ for all $W \in Y$.

Lemma 10.4. Let there be given an almost complex manifold $M$, a point $y$ in $M$, a real manifold $\Sigma$, a neighborhood $U^{\prime}$ of 0 in $T_{y} M$, as well as $C^{\infty}$ mappings $\Phi: \Sigma \rightarrow T_{y} M$ and $F: U^{\prime} \rightarrow M$ such that $\Phi$ is a totally real immersion/embedding of $\Sigma$ in the complex vector space $T_{y} M$, while $F(0)=y$ and $d F_{0}$ is the identity mapping of $T_{y} M$. If, in addition, the image $Y$ of the Gauss mapping (10.1), with $V=T_{y} M$, is compact, and $\varepsilon: T_{y} M \rightarrow T_{y} M$ denotes the multiplication by $\varepsilon \in \mathbf{R}$, then, for some neighborhood $U$ of 0 in $T_{y} M$ contained in $U^{\prime}$, and all sufficiently small $\varepsilon>0$, the composite $F \circ \varepsilon \circ \Phi: \Phi^{-1}\left(\varepsilon^{-1} U\right) \rightarrow M$ is a totally real immersion/embedding in $M$ of the open subset $\Phi^{-1}\left(\varepsilon^{-1} U\right)$ of $\Sigma$.

Proof. Let $U^{\prime} \ni x \mapsto J(x) \in \operatorname{Hom}_{\mathbf{R}}(V, V)$, with $V=T_{y} M$, be the $F$-pullback to $U^{\prime}$ of the original almost complex structure in $M$, and let $U \subset U^{\prime}$ be a neighborhood of 0 in $V$ such that $J(x) \in \Omega$ for all $x \in U$, with $\Omega$ obtained by applying Lemma 10.3 to our $Y$ and $J=J(0)$. Obviously, $d \varepsilon_{v}(W)=W$ whenever $\varepsilon \neq 0, v \in V$ and $W$ is a real vector subspace of $V=T_{v} V=T_{\varepsilon v} V$. Hence $\varepsilon \circ \Phi: \Phi^{-1}\left(\varepsilon^{-1} U\right) \rightarrow U$ is a totally real immersion/embedding relative to the almost complex structure in the receiving manifold $U$, pulled back from $M$ via $F$.

Remark 10.5. Lemma 10.4 becomes particularly simple when $\Sigma$ is compact (and hence so is $Y$ ), as one then has $\varepsilon(\Phi(\Sigma)) \subset U$ for sufficiently small $\varepsilon>0$, and so $F \circ \varepsilon \circ \Phi$ is a totally real immersion/embedding of $\Sigma$ in $M$.

\section{IMMERSIONS OF SPHERES}

The immersions described in Proposition 11.2 were found by Whitney 34 and Weinstein [29]. Gromov [19] showed that no totally real embedding $S^{n} \rightarrow \mathbf{C}^{n}$ exists unless $n \in\{1,3\}$. (This was independently proved by Stout and Zame 28]; cf. also [31, p. 430] and [21.) For $n=3$ such embeddings do exist, which is a result of Ahern and Rudin [3]; see also [12] and [10]. On the other hand, every embedding $S^{1} \rightarrow \mathbf{C}$ is totally real.

Lemma 11.1. Let || be a fixed Euclidean norm in a totally real subspace $W$ of a complex vector space $V$ with $\operatorname{dim}_{\mathbf{R}} W=\operatorname{dim}_{\mathbf{C}} V$, and let $S$ denote the sphere of some radius $a>0$ in $W$, centered at 0 . Furthermore, let $K \subset \mathbf{C} \backslash\{0\}$ be an embedded $C^{\infty}$ curve with $0 \notin K+K$, where $K+K=\{b+c: b, c \in K\}$. Then the mapping $f: K \times S \rightarrow V$, given by $f(c, v)=c v$, is a totally real embedding.

Proof. The differential of $f$ at $(c, v)$ sends $(\dot{c}, \dot{v}) \in T_{c} K \times T_{v} S \subset \mathbf{C} \times W$ to $\dot{c} v+c \dot{v}$, and so it transforms an $\mathbf{R}$-basis of $T_{c} K \times T_{v} S$, each of whose vectors $(\dot{c}, \dot{v})$ has either $\dot{c}=0$ or $\dot{v}=0$, onto a $\mathbf{C}$-basis of $V$. Thus, $f$ is a totally real immersion (see Example 9.1). Injectivity of $f$ follows since the relation $c v=b w$ with $b, c \in K$ and $v, w \in S$ implies $v=c^{-1} b w$, and so, as $W$ is totally real and $|v|=|w|=a$, we have $b= \pm c$ (cf. Example 9.1 again), while $b \neq-c$ in view of the assumption that $0 \notin K+K$.

Any fixed Euclidean norm in a totally real subspace $W$ of a complex vector space $V$ with $\operatorname{dim}_{\mathbf{R}} W=\operatorname{dim}_{\mathbf{C}} V=n$ gives rise to the group $\operatorname{SO}(W) \approx \operatorname{SO}(n)$ of all 
orientation-preserving linear isometries of $W$, with the inclusion $\mathrm{SO}(W) \subset \mathrm{GL}(V)$ obtained by extending operators $W \rightarrow W$ complex-linearly to $V$.

Proposition 11.2. Let $W$ and $L$ be a totally real subspace and a complex subspace of a complex vector space $V$ with $W \cap L \neq\{0\}, \operatorname{dim}_{\mathbf{R}} W=\operatorname{dim}_{\mathbf{C}} V=n$ and $\operatorname{dim}_{\mathbf{C}} L=1$. Next, let $\Gamma \subset L$ be a compact set such that $0 \in \Gamma$ and $\Gamma \backslash\{0\}$ is a 1-dimensional $C^{\infty}$ submanifold of $L$ with $v+v^{\prime} \neq 0$ whenever $v, v^{\prime} \in \Gamma \backslash\{0\}$, while the intersection of $\Gamma$ with some neighborhood of 0 in $L$ consists of two nonparallel real-line segments emanating from 0 . Finally, let $Q=\mathrm{SO}(W) \Gamma$, so that $Q$ is the set of all $A x$ with $A \in \mathrm{SO}(W)$ and $x \in \Gamma$, where $\mathrm{SO}(W) \subset \mathrm{GL}(V)$ is defined as above for any fixed Euclidean norm || in $W$.

Then $Q$ is a totally real $n$-sphere immersed in $V$. It has just one self-intersection in the form of a double point at 0 , and its two tangent spaces $T, T^{\prime}$ at 0 are related by $T^{\prime}=z T$ for some $z \in \mathbf{C} \backslash \mathbf{R}$.

Proof. Fix $u \in W \cap L \backslash\{0\}$. Now $\Gamma \backslash\{0\}=K u$ for some embedded $C^{\infty}$ curve $K \subset \mathbf{C} \backslash\{0\}$ with $0 \notin K+K$, which approaches 0 along two real-line segments $(0,1) b$ and $(0,1) c$, where $b, c \in \mathbf{C} \backslash\{0\}$ and $b / c \notin \mathbf{R}$. For the sphere $S$ of radius $|u|$ in $W$, centered at 0 , we have $Q \backslash\{0\}=K S$, as $Q \backslash\{0\}=\operatorname{SO}(W) K u=$ $K \mathrm{SO}(W) u$. Thus, in view of Lemma 11.1, $Q \backslash\{0\}$ is a totally real submanifold of $V$, diffeomorphic to $K \times S$, that is, to $S^{n}$ minus two points. However, because of how $K$ approaches 0 in $\mathbf{C}$, a neighborhood of 0 in $Q$ is the union of two open $n$-balls centered at 0 in the totally real subspaces $T=b W$ and $T^{\prime}=c W$ spanned by $\mathrm{SO}(W) b u$ and $\mathrm{SO}(W) c u$.

As an obvious consequence of Propositions 10.1 and 11.2, we obtain

Corollary 11.3. Given an almost complex manifold $M$ with $\operatorname{dim}_{\mathbf{C}} M=n \geq 2$, a point $y \in M$, and a neighborhood $U$ of $y$ in $M$, there exists a totally real immersion of the $n$-sphere in $U$ which has a double point with a transverse selfintersection at $y$, and no other multiple points.

\section{Totally REAL BLOW-UPS}

Let $M^{\prime}$ be the complex manifold, diffeomorphic to $M \# \overline{\mathbf{C P}^{n}}$, obtained by blowing up a point in a given complex manifold $M$ with $n=\operatorname{dim}_{\mathbf{C}} M$.

Totally real immersions/embeddings in $M$ lead to totally real immersions/embeddings in $M^{\prime}$. We discuss three such constructions, two of which are summarized in the following lemma; the third one is presented, for $n=2$ only, in Section 13 (see Remark 13.1).

Lemma 12.1. Given a complex manifold $M$, a point $y \in M$, and a $k$-dimensional totally real submanifold $\Sigma \subset M$ which is closed as a subset of $M$ and carries the subset topology, let the complex manifold $M^{\prime}=M \# \overline{\mathbf{C P}^{n}}$, where $n=\operatorname{dim}_{\mathbf{C}} M$, be the result of blowing up $y$ in $M$.

(a) If $y \notin \Sigma$, then $\Sigma$ is also totally real as a submanifold of $M^{\prime}$.

(b) If $y \in \Sigma$, then the closure $\hat{\Sigma}$, in $M^{\prime}$, of the preimage of $\Sigma \backslash\{y\}$ under the blow-down projection $M^{\prime} \rightarrow M$, is a totally real submanifold of $M^{\prime}$, diffeomorphic to the manifold $\Sigma^{\prime} \approx \Sigma \# \mathbf{R P}^{k}$ obtained by the real blow-up of $y$ in $\Sigma$. 
(c) If $n=k=2$ and $y \in \Sigma$, while $\mathbf{P} \subset M^{\prime}$ is the divisor created by the blow-up, then the surface $\hat{\Sigma}$ in (b) can be deformed, by arbitrarily small isotopies supported in any given open subset of $M^{\prime}$ containing $\mathbf{P}$, to a surface embedded in $M^{\prime}$ and having a single, transverse intersection with the divisor $\mathbf{P}$.

Proof. (a) is obvious. For (b), let us fix a point $y^{\prime} \in \pi^{-1}(y) \subset \Sigma^{\prime}$, where $\pi: \Sigma^{\prime} \rightarrow \Sigma$ is the real blow-down projection. We may choose holomorphic coordinates $z_{a}$ in $M, a=1, \ldots, n$, and $C^{\infty}$ coordinates $x_{j}$ in $\Sigma, j=1, \ldots, k$, both defined near $y$, such that $z_{a}=x_{j}=0$ and $\partial z_{a} / \partial x_{j}=\delta_{a j}$ at $y$ for all $a, j$, while $y^{\prime}$, as a real line through 0 in $T_{y} \Sigma$, is tangent to the $x_{1}$ coordinate axis. (This is easily achieved by an affine coordinate change; cf. Example 9.1.) In suitable local coordinates $x, \xi_{2}, \ldots, \xi_{k}$ and $z, \zeta_{2}, \ldots, \zeta_{n}$ for $\Sigma^{\prime}$ and $M^{\prime}$, the blow-down projections $\Sigma^{\prime} \rightarrow \Sigma$ and $M^{\prime} \rightarrow M$ are given by $\left(x_{1}, \ldots, x_{k}\right)=\left(x, x \xi_{2}, \ldots, x \xi_{k}\right)$ and $\left(z_{1}, \ldots, z_{n}\right)=\left(z, z \zeta_{2}, \ldots, z \zeta_{n}\right)$, with $x \in \mathbf{R}$ and $z \in \mathbf{C}$ both varying in neighborhoods of 0 . The integral form of the first-order Taylor formula now gives $z_{a}=$ $\sum_{j=1}^{k} x_{j} h_{a j}\left(x_{1}, \ldots, x_{k}\right)$ for some $C^{\infty}$ functions $h_{a j}$ with $h_{a j}=\partial z_{a} / \partial x_{j}=\delta_{a j}$ at $x_{1}=\ldots=x_{k}=0$. In terms of $x, \xi_{2}, \ldots, \xi_{k}$ this becomes $z=z_{1}=x \mu\left(x, \xi_{2}, \ldots, \xi_{k}\right)$ for a $C^{\infty}$ function $\mu$ with $\mu\left(0, \xi_{2}, \ldots, \xi_{k}\right)=1$. Similarly, $z_{a} / x$ is, for each $a=2, \ldots, n$, a $C^{\infty}$ function of $x, \xi_{2}, \ldots, \xi_{k}$ (where $x$ varies around 0 in $\mathbf{R}$ ). Hence so is $\zeta_{a}=z_{a} / z=z_{a} /(x \mu)$, while $\left(z, \zeta_{2}, \ldots, \zeta_{n}\right) \rightarrow\left(0, \xi_{2}, \ldots, \xi_{k}, 0, \ldots, 0\right)$ as $x \rightarrow 0$, so that $\partial \zeta_{a} / \partial \xi_{j}=\delta_{a j}$ wherever $x=0$. The inclusion mapping $\Sigma \backslash\{y\} \rightarrow M \backslash\{y\}$ thus has a $C^{\infty}$ extension $f: \Sigma^{\prime} \rightarrow M^{\prime}$ represented by our assignment $\left(x, \xi_{2}, \ldots, \xi_{k}\right) \mapsto\left(z, \zeta_{2}, \ldots, \zeta_{n}\right)$, which is again injective, as it acts by $\left(0, \xi_{2}, \ldots, \xi_{k}\right) \mapsto\left(0, \xi_{2}, \ldots, \xi_{k}, 0, \ldots, 0\right)$ on the added $\mathbf{R} P^{k-1}$ given by the equation $x=0$.

At $x=0$ we have $\mu=1$ and hence $\partial \mu / \partial \xi_{j}=0$, while, as we just saw, $\partial \zeta_{a} / \partial \xi_{j}=\delta_{a j}$ if $x=0$. On the other hand, the relation $z=x \mu$ gives $\partial z / \partial x=$ $\mu=1$ and $\partial z / \partial \xi_{j}=0$ when $x=0$. Consequently, at points with $x=0$, the matrix $\mathfrak{A}=\left[\partial \zeta_{a} / \partial \xi_{j}\right]$ with $1 \leq a \leq n$ and $1 \leq j \leq k$ (where $\xi_{1}=x, \zeta_{1}=z$ ) has a nonzero $k \times k$ subdeterminant obtained by restricting $a$ to $\{1, \ldots, k\}$. Therefore, $\operatorname{rank} \mathfrak{A}=k$, and so $f$ is a totally real immersion (see Example 9.1), which proves assertion (b).

Now let $n=k=2$, and let $f: \Sigma^{\prime} \rightarrow M^{\prime}$ be the totally real embedding with $f\left(\Sigma^{\prime}\right)=\hat{\Sigma}$, described above. Thus, $\Gamma=\hat{\Sigma} \cap \mathbf{P}$ is the $f$-image of the circle $\mathbf{R P}^{1}$ in $\Sigma^{\prime}$ created by the real blow-up of $y \in \Sigma$. A tubular neighborhood $U$ of $\Gamma$ in $\hat{\Sigma}$ is diffeomorphic to the Möbius strip, so that we may fix $\varepsilon>0$ and a two-fold covering map $F: S^{1} \times(-\varepsilon, \varepsilon) \rightarrow U$ invariant under the involution of $S^{1} \times(-\varepsilon, \varepsilon)$ sending $(z, t)$ to $(-z,-t)$, with $S^{1}=\{z \in \mathbf{C}:|z|=1\}$. The push-forward under $F$ of the standard unit vector field on $S^{1} \times(-\varepsilon, \varepsilon)$ tangent to the $(-\varepsilon, \varepsilon)$ factor is a double-valued vector field $\pm v$ tangent to $U$, defined only up to a sign. If $\varepsilon$ is made sufficiently small and a Riemannian metric is chosen on $M$, the union of suitable short geodesic segments emanating from points of $U$ in the direction of $\pm i v$ is a 3 -dimensional open submanifold $N$ of $M$, and $F$ has an extension to a two-fold covering map $H: S^{1} \times D_{\varepsilon} \rightarrow N$, where $D_{\varepsilon}=\{w \in \mathbf{C}:|w|<\varepsilon\}$, such that $H$ is invariant under the involution $(z, w) \mapsto(-z,-w)$. Making $\varepsilon$ even smaller, if necessary, we may also assume that $H\left(S^{1} \times D_{\varepsilon}\right)$ intersects $\mathbf{P}$ only along $\Gamma$. (In fact, since $\hat{\Sigma}$ is totally real, at each point of $\Gamma$ the complex line spanned by $\pm v$ has a trivial intersection with the tangent complex line of $\mathbf{P}$.) 
Finally, let us fix a $C^{\infty}$ function $\varphi:[0, \infty) \rightarrow \mathbf{R}$ equal to 1 near 0 and vanishing outside the interval $\left(-\varepsilon^{2} / 2, \varepsilon^{2} / 2\right)$. We now obtain the required small deformations of $\hat{\Sigma}$ by replacing $U \subset \hat{\Sigma}$ with the $H$-image of the involution-invariant surface $\left\{\left(z, t+\operatorname{ri\varphi }\left(t^{2}\right) \operatorname{Re} z\right): z \in S^{1}, t \in(-\varepsilon, \varepsilon)\right\}$, depending on a real parameter $r$ close to 0 , and transverse to the curve $S^{1} \times\{0\}$.

In the following obvious corollary, by the nonorientable closed surface of genus $s \geq 1$ we mean, as usual, the connected sum $s \mathbf{R} \mathbf{P}^{2}$ of $s$ copies of $\mathbf{R P}^{2}$.

Corollary 12.2. The surface $3 \mathbf{R} \mathbf{P}^{2}=\mathbf{R} \mathbf{P}^{2} \# \mathbf{R} \mathbf{P}^{2} \# \mathbf{R} P^{2}$ admits totally real embeddings in $\mathbf{C P}^{2} \# m \overline{\mathbf{C P}^{2}}$ for all $m \geq 1$.

More generally, for any integers $m, s$ with $m \geq s-2 \geq 0$, the nonorientable closed surface $s \mathbf{R P}^{2}$ of genus $s$ admits a totally real embedding in the complex surface $\mathbf{C P}^{2} \# m \overline{\mathbf{C P}^{2}}$ obtained by blowing up the points of a suitable m-element set in $\mathrm{CP}^{2}$.

This is clear if one blows up $m$ distinct points of $\mathbf{C P}^{2}$, of which $s-2$ lie in a given totally real torus embedded in $\mathbf{C}^{2} \subset \mathbf{C P}^{2}$ (cf. (v) in Section 9), and then uses Lemma 12.1 .

\section{Removability of COMPlex POINTS By BLOW-UP}

Given a point $y$ of a real surface $\Sigma$ embedded in a complex surface $M$, we will say that $\Sigma$ contains $y$ as a complex point removable by blow-up if, for some neighborhood $U$ of $y$ in $\Sigma$ and some totally real $C^{\infty}$ submanifold $U^{\prime}$ of the complex surface $M^{\prime}$ obtained from $M$ by blowing up the point $y$, the blow-down projection $\pi: M^{\prime} \rightarrow M$ maps $U^{\prime}$ diffeomorphically onto $U$. This amounts to requiring that $\pi^{-1}(x)$ have a limit $y^{\prime} \in M^{\prime}$ as $x \in U \backslash\{y\}$ approaches $y$, and that $U^{\prime}=\left\{y^{\prime}\right\} \cup \pi^{-1}(U \backslash\{y\})$ be a totally real $C^{\infty}$ submanifold of $M^{\prime}$ transverse, at $y^{\prime}$, to the divisor $\pi^{-1}(y)$. Note that $y$ then must actually be an (isolated) complex point of $\Sigma$, since $T_{y} \Sigma$ coincides with the image of the differential of $\pi$ at $y^{\prime}$ (which is the complex line in $T_{y} M$ corresponding to $y^{\prime}$ ), while $\pi: M^{\prime} \backslash \pi^{-1}(y) \rightarrow M \backslash\{y\}$ is a biholomorphism (and so $U \backslash\{y\}=\pi\left(U^{\prime} \backslash\left\{y^{\prime}\right\}\right.$ ) is totally real in $M$ ).

Remark 13.1. Removability by blow-up leads to our third blow-up procedure. Namely, let a real surface $\Sigma$ embedded in a complex surface $M$ be totally real except for a finite number of complex points removable by blow-up, and let $M^{\prime}$ be the complex surface obtained from $M$ by blowing up those points. Then $M^{\prime}$ contains a totally real embedded surface $\Sigma^{\prime}$, which the blow-down projection $M^{\prime} \rightarrow M$ maps diffeomorphically onto $\Sigma$.

Example 13.2. Let $\Sigma=\{(z, w) \in U \times \mathbf{C}: w=h(z)\}$ be the graph of a $C^{\infty}$ function $h: U \rightarrow \mathbf{C}$ defined on a neighborhood $U$ of 0 in $\mathbf{C}$, and let $y=(0, h(0))$. Then the following two conditions are equivalent:

(a) $\Sigma$ treated as a real surface embedded in $M=\mathbf{C}^{2}$ contains $y$ as a complex point removable by blow-up, while $\Sigma \backslash\{y\}$ is totally real;

(b) $h(z)=h(0)+z \varphi(z)$ for all $z \in U$, where $\varphi: U \rightarrow \mathbf{C}$ is a $C^{\infty}$ function satisfying at each point the inequality $\varphi_{\bar{z}} \neq 0$ of Example 9.3.

In fact, let $M^{\prime}$ be the complex surface obtained from $\mathbf{C}^{2}$ by blowing up $y$. The blow-down projection $\pi: M^{\prime} \rightarrow \mathbf{C}^{2}$ is given by $(\zeta, \eta) \mapsto(z, w)=(\zeta, \zeta \eta+h(0))$ in suitable holomorphic local coordinates $(\zeta, \eta)$ for $M^{\prime}$. The relation $w=h(z)$ for 
$z \neq 0$ now reads $\eta=[h(\zeta)-h(0)] / \zeta$ whenever $\zeta \neq 0$. Thus, our claim follows from Example 9.3 note that a limit $L$ of $[h(\zeta)-h(0)] / \zeta$ as $\zeta \rightarrow 0$, if it exists, must be finite: using real values of $\zeta$ we get $L=h_{x}(0)$, with $h_{x}=\partial h / \partial x$ and $x=\operatorname{Re} z$.

Example 13.3. The graph surface $\Sigma=\{(z, w) \in U \times \mathbf{C}: w=z \bar{z}\} \subset \mathbf{C}^{2}$ is a paraboloid of revolution in the real subspace $\mathbf{C} \times \mathbf{R}$, and $y=(0,0)$ is its unique complex point, as well as a complex point removable by blow-up. This is clear from Example 13.2 with $U=\mathbf{C}$ and $h(z)=z \bar{z}$.

Example 13.4. The embedded 2-sphere $S \subset \mathbf{C P}^{2}$ given by $a \bar{a}=b \bar{c}$ in the homogeneous coordinates $[a, b, c]$ has just two complex points, $x=[0,0,1]$ and $y=[0,1,0]$, both removable by blow-up. This is clear from Example 13.3. if one removes $x$ (or $y$ ) from $S$, by setting $b=1$ (or $c=1$ ), one gets the paraboloid $c=|a|^{2}$ (or $b=|a|^{2}$ ) in the $a c$-plane (or, $a b$-plane).

Example 13.5. In $\mathbf{C}^{2}$ with the coordinates $(a, b)$, the 2-sphere $S$ of radius $R>0$, given by $|a|^{2}+|b|^{2}=R^{2}$ and $\operatorname{Im} b=0$, has just two complex points $x^{ \pm}=(0, \pm R)$, both removable by blow-up. In fact, $S \backslash\left\{x^{+}, x^{-}\right\}$is totally real since $\mathbf{C} \times \mathbf{R}$ contains only one complex-line direction: that of the $z$ axis $\mathbf{C} \times\{0\}$. Removability of $x^{ \pm}$is clear from Example 13.2 in the new coordinates $(z, w)=(a, R \mp b)$, the components of $x^{ \pm}$are $(z, w)=(0,0)$, while $S$ is, near $x^{ \pm}$, the graph of $w=h(z)$ for $h(z)=R-\sqrt{R^{2}-|z|^{2}}$. Next, $\varphi(z)=h(z) / z$ equals $\bar{z} F(z \bar{z})$, where $F(s)=\left[R-\left(R^{2}-s\right)^{1 / 2}\right] / s$ is obviously real-analytic at $s=0$. Finally, $\varphi_{\bar{z}}(0)=F(0)=R / 2>0$.

\section{Deformations involving COMPlex points}

The definition of removability by blow-up given in Section 13 has an immediate extension to the case of immersions. Namely, if $f$ is an immersion of a real surface $\Sigma$ in a complex surface $M$, by a complex point of $f$ removable by blow-up we mean any $x \in \Sigma$ such that $f(x)$ is a complex point, removable by blow-up, for the surface $f\left(\Sigma^{\prime}\right) \subset M$, where $\Sigma^{\prime}$ is some connected neighborhood of $x$ in $\Sigma$ with the property that $f$ restricted to $\Sigma^{\prime}$ is injective. As in Section [13, $x$ then is a complex point of $f$, that is, $d f_{x}\left(T_{x} \Sigma\right)$ forms a complex line in $T_{f(x)} M$. Cf. Example 9.4.

The following lemma allows us to modify an immersion $f$ by moving the images of any number $s$ of its complex points $x_{j}$, removable by blow-up, to arbitrary prescribed locations $y_{j}$. This is achieved by replacing the $f$-image of a small neighborhood of each $x_{j}$ with a thin protrusion or "tentacle" reaching all the way to $y_{j}$.

Lemma 14.1. Given $s$ distinct points $x_{1}, \ldots, x_{s}$ in a real surface $\Sigma$, a complex surface $M$, points $y_{1}, \ldots, y_{s} \in M$, and an immersion $f: \Sigma \rightarrow M$ such that $x_{1}, \ldots, x_{s}$ are complex points of $f$ removable by blow-up, there exists an immersion $f^{\prime}: \Sigma \rightarrow M$ homotopic to $f$, with the same complex points as $f$, for which $x_{1}, \ldots, x_{s}$ are complex points removable by blow-up and $f^{\prime}\left(x_{j}\right)=y_{j}$ for $j=1, \ldots, s$.

If, in addition, $y_{1}, \ldots, y_{s}$ are all distinct and lie in $M \backslash f(\Sigma)$, the above assertion also remains valid when instead of an immersion one speaks, in both instances, of an embedding whose image is closed as a subset of $M$. 
Proof. We may assume that $s=1$, since the case $s \geq 2$ will then follow via induction on $s$. Namely, $f$ can be deformed to $f^{\prime}$ in two stages: first, using the inductive assumption that our claim holds for $x_{j}, y_{j}, j=2, \ldots, s$, with $M$ replaced by $M \backslash\left\{y_{1}\right\}$ if $f$ is an embedding; then, applying our claim for $s=1$ to the resulting new immersion and the points $x_{1}, y_{1}$.

With $s=1$, writing $x, y$ for $x_{1}, y_{1}$ and $p=f(x)$, let us also assume that

(*) some biholomorphism $(z, w)$ maps an open set in $M$ containing $p$ and $y$ onto a product $D \times D^{\prime}$ of disks around 0 in $\mathbf{C}$ with $1 \in D^{\prime}$, sending $p$ to $(0,0)$ and $y$ to $(0,1)$, while, if $f$ is an embedding and $f(\Sigma)$ is a closed set in $M$, the $(z, w)$-preimage of $\{0\} \times(0,1]$ does not intersect $f(\Sigma)$.

To prove our assertion for $s=1$, under the hypothesis $(*)$, we may further assume $d z$ at $p$, restricted to $d f_{x}\left(T_{x} \Sigma\right)$, to be nonzero. (This is achieved by replacing $(z, w)$ with $(z+\mu w(w-1), w)$ for $\mu \in \mathbf{R}$ close to 0 , and making $D$ smaller if necessary.) Let $U$ now be a neighborhood of $x$ in $\Sigma$ such that $f: U \rightarrow M$ is a homeomorphic embedding. Thus, $(z, w)$ maps some neighborhood of $p$ in $f(U)$ onto a graph surface $w=h(z)$ as in Example 13.2, with $h(0)=0$. Next, if $D$ is made even smaller, we may in addition require that $h$ be defined on the whole disk $D$, and (after the coordinate $z$ has been replaced by $e^{i \theta} z$ for a suitable $\theta \in \mathbf{R}$ ), that also $\varphi_{\bar{z}}(0) \notin \mathbf{R}$, for $\varphi$ appearing in Example 13.2. (Notation of Example 9.3.) Hence $\varphi_{\bar{z}}(z) \notin \mathbf{R}$ whenever $z \in \mathbf{C}$ and $|z|<\varepsilon$ for some fixed $\varepsilon>0$ that is less than the radius of $D$. Let us now fix $\delta \in(0, \varepsilon)$ and a $C^{\infty}$ function $\alpha: \mathbf{R} \rightarrow[0,1]$ with $\alpha=1$ on $(-\infty, \delta]$ and $\alpha=0$ on $[\varepsilon, \infty)$. Our deformation of $f$ consists in replacing the graph of $h: D \rightarrow D^{\prime}$ by that of the function $\tilde{h}: D \rightarrow D^{\prime}$ with $\tilde{h}(z)=h(z)+\alpha(r)$ for $z \in D$ and $r=|z|$. This is clear from Example 13.2, as $\tilde{h}(z)=\tilde{h}(0)+z \tilde{\varphi}(z)$ with $\tilde{\varphi}(z)=\varphi(z)+[\alpha(r)-1] / z$, so that the relations $2 \tilde{\varphi}_{\bar{z}}=2 \varphi_{\bar{z}}+r^{-1} d \alpha / d r$ (immediate since $z_{\bar{z}}=0$ and $\left(r^{2}\right)_{\bar{z}}=z$, that is, $\left.2 r_{\bar{z}}=z / r\right)$, and $\varphi_{\bar{z}}(z) \notin \mathbf{R}$ whenever $|z|<\varepsilon$ (see above) give $\tilde{\varphi}_{\bar{z}} \neq 0$ everywhere in $D$, as required in Example 13.2(b).

Finally, to obtain our assertion for $s=1$, it suffices to prove it, as we just did, under the additional assumption $(*)$. In fact, as $M$ is connected, there exist $p_{0}, p_{1}, \ldots, p_{m} \in M$ with $p_{0}=f(x), p_{m}=y$ and such that $(*)$ holds if $p, y$ are replaced by $p_{l-1}, p_{l}$, for any $l=1, \ldots, m$. Specifically, $f$ can be modified $m$ times in a row, leading to successive totally real immersions $f_{0}, f_{1}, \ldots, f_{m}$ of $\Sigma$ in $M$, with $f_{l}(x)=p_{l}$ for $l=0, \ldots, m$, all homotopic to $f_{0}=f$, and we may set $f^{\prime}=f_{m}$. The final clause of the lemma now follows as well, provided that one chooses $p_{1}, \ldots, p_{m-1}$ and the corresponding $m$ biholomorphisms in $(*)$ more carefully, requiring the $m$ preimages of $\{0\} \times(0,1]$ to be disjoint except for the endpoints shared by the $l$ th and $(l+1)$ st preimage, $l=1, \ldots, m$, and not to intersect $f(\Sigma)$ except at $p=f(x)$. (To guarantee such disjointness properties in the case of an embedding, the disk $D$ mentioned in the last paragraph needs to be chosen sufficiently small at each stage.) This completes the proof.

Proof of Theorem 2.15. Assertion (b) is obvious from Lemma 12.1. To establish (a), let $s \geq 2$ and let $y_{1}, y_{2} \in M$ be any two of the blown-up points $\left(y_{1} \neq y_{2}\right)$. A biholomorphic identification of an open set in $M$ with a neighborhood of $(0,0)$ in $\mathbf{C}^{2}$ allows us to treat a small round sphere $S$ of Example 13.5 as a real surface in $M \backslash\left\{y_{1}, y_{2}\right\}$ having only two complex points $x^{ \pm}$, both removable by blow-up. 
Now (a) follows from the final clause of Lemma 14.1 applied to $s=1$, the inclusion mapping $f: \Sigma \rightarrow M$ of the sphere $\Sigma=S$, our $y_{1}, y_{2}$, and $x_{1}=x^{+}, x_{2}=x^{-}$.

\section{OTHER IMMERSED TWO-SPHERES}

The main result of this section, Corollary [15.3, establishes the existence of totally real immersions $f: S^{2} \rightarrow \mathbf{C P}^{2} \# m \overline{\mathbf{C P}^{2}}$, for $m \in\{1,3\}$, with some special properties. We use such immersions in Sections 30 and 36 .

Lemma 15.1. Let there be given the total space $M$ of a holomorphic line bundle $\mathcal{L}$ over a complex curve $\Sigma$, a holomorphic section $\phi$ of $\mathcal{L}$, and a $C^{\infty}$ function $F: \Sigma \rightarrow \mathbf{R}$. Then, for the product section $F \phi$ treated as an embedding $f$ of $\Sigma$ in the complex surface $M$,

(i) the complex points of $f$ are those $x \in \Sigma$ at which $\phi=0$ or $d F=0$. A complex point $x$ of $f$ is removable by blow-up, cf. Section 13, if

(a) $x$ is a simple zero of $\phi$ and $d F \neq 0$ at $x$, or

(b) $\phi(x) \neq 0$, and there exists a holomorphic coordinate $z$ on a neighborhood of $x$ in $\Sigma$ with $z=0$ at $x$ and $F=F(x)+z \varphi$ for some $C^{\infty}$ function $\varphi$ such that $\varphi(x)=0$.

Proof. Given $x \in \Sigma$, let us choose a holomorphic coordinate $z$ on a neighborhood $U$ of $x$ in $\Sigma$, with $z=0$ at $x$, and a local holomorphic section $\psi$ trivializing $\mathcal{L}$ on $U$. In the resulting holomorphic local coordinates $(z, w)$ for $M$, the image $f(\Sigma)$ becomes a graph surface $w=h(z)$, with $h=\sigma F$ for the holomorphic function $\sigma$ such that $\phi=\sigma \psi$ on $U$. Example 9.3 now yields (i), since $\sigma_{\bar{z}}=0$, and so $h_{\bar{z}}=\sigma F_{\bar{z}}$ vanishes only where $\sigma=0$ (that is, $\phi=0$ ) or $F_{\bar{z}}=0$ (which, as $F$ is real-valued, amounts to $d F=0)$. Similarly, Example 13.2 gives removability of $x$ by blow-up, both in case (ii) (as $\sigma / z$ is then holomorphic on $U$ and $F_{\bar{z}}(x) \neq 0$, so that $(\sigma F / z)_{\bar{z}} \neq 0$ at $x$ ) and in case (iii) (since we may choose $\psi=\phi$, that is, $\sigma=1)$. This completes the proof.

Proposition 15.2. An oriented 2-sphere $\Sigma$ admits an immersion $f$ in $\mathbf{C P}^{2}$ such that, for some three-element set $Y \subset \Sigma$,

(a) $f_{*}[\Sigma]$ is a generator of $H_{2}\left(\mathbf{C P}^{2}, \mathbf{Z}\right)$,

(b) $Y$ consists of three complex points of $f$ removable by blow-up,

(c) the immersion $f: \Sigma \backslash Y \rightarrow \mathbf{C P}^{2}$ is totally real.

Furthermore, such $f$ and $Y$ can be chosen so that either

(i) $f$ is an embedding, or

(ii) $Y$ is the $f$-preimage $f^{-1}(y)$ of a point $y \in \mathbf{C P}^{2}$.

Proof. To obtain (a)-(c) and (i), we define $M \subset \mathbf{C P}^{2}$ to be the complement of a point in $\mathrm{CP}^{2}$, so that $M$ is biholomorphic to the total space of the dual tautological line bundle $\mathcal{L}$ of a projective line $\Sigma \subset M$. Our claim is now obvious from Lemma 15.1 if we choose a holomorphic section $\phi$ of $\mathcal{L}$ with a unique, simple zero and a $C^{\infty}$ function $F: \Sigma \rightarrow \mathbf{R}$ having just two critical points, such that either critical point $x$ satisfies condition (b) in Lemma 15.1 Specifically, treating $\Sigma$ as the Riemann sphere $\mathbf{C} \cup\{\infty\}$, we may set $F=z \bar{z} /(z \bar{z}+1)$ for $z \in \mathbf{C} \subset \Sigma$. The role of the coordinate $z$ in Lemma 15.1(b) is now played by $z$ at $x=0 \in \mathbf{C}$, and by $1 / z$ at $x=\infty$, while $F$ becomes a standard height function if we identify $\mathbf{C} \cup\{\infty\}$, via the stereographic projection, with a sphere in $\mathbf{R}^{3}=\mathbf{C} \times \mathbf{R}$. 
To realize (a)-(c) and (ii), we fix $f$ and $Y$ with (a)-(c) and (i), and then replace $f$ by $f^{\prime}$ chosen as in Lemma 14.1 for $M=\mathbf{C P}^{2}$, our $f, \Sigma$, and $s=3$, with $x_{j} \in Y$ and $y_{j}=y, j=1,2,3$.

Blowing up all points of $Z=f(Y)$ in Proposition 15.2, we obtain the following obvious consequence.

Corollary 15.3. Given $m \in\{1,3\}$ and any m-element set $Z \subset \mathbf{C P}^{2}$, let $M$ be the complex surface obtained from $\mathbf{C P}^{2}$ by blowing up all points of $Z$. The oriented 2 -sphere $S^{2}$ then admits a totally real immersion $f: S^{2} \rightarrow M$ with $f_{*}\left[S^{2}\right] \cdot[L]=1$ for any projective line $L \subset \mathbf{C P}^{2} \backslash Z$ treated as a submanifold of $M$, where - is the intersection form in $\mathrm{H}_{2}(M, \mathbf{Z})$. If, in addition, $m=3$, we may choose $f$ to be an embedding.

\section{Removal OF transVERSE INTERSECTIONS}

In this section we describe how, using infinitely many homotopically differrent surgeries, one can remove a transverse self-intersection of a totally real surface immersed in an almost complex surface.

Let $P, Q$ be oriented real planes with $P \cap Q=\{0\}$ in a complex plane $V$. We define the sign of the (transverse) intersection of $P$ and $Q$ to be positive, or negative, if the direct-sum orientation of $V=P \oplus Q$ does or, respectively, does not agree with the standard orientation of $V$ described at the beginning of Section 5 , The sign remains the same if $P$ and $Q$ are switched, or their orientations are both reversed. This allows us to extend the concept of the sign to the case of a double point $y=f(x)=f\left(x^{\prime}\right)$ representing a transverse self-intersection of an immersion $f: \Sigma \rightarrow M$, in an almost complex surface $M$, of a real surface $\Sigma$ which is orientable (though not necessarily oriented).

Lemma 16.1. Let $P, Q$ and $W$ be real vector spaces of dimension $n \geq 2$, and let $\gamma: P \times Q \rightarrow W$ be a bilinear mapping such that $\gamma(u, v) \neq 0$ whenever $u \in P \backslash\{0\}$ and $v \in Q \backslash\{0\}$. If $u_{j}$ and $v_{k}$ are fixed bases of $P$ and $Q$, and $\mathfrak{T}_{\xi}$ is the $n \times n$ matrix with the entries $\xi\left(\gamma\left(u_{j}, v_{k}\right)\right)$, where $\xi \in W^{*}$ and $j, k \in\{1, \ldots, n\}$, then either $\operatorname{det} \mathfrak{T}_{\xi}>0$ for all $\xi \in W^{*} \backslash\{0\}$ or $\operatorname{det} \mathfrak{T}_{\xi}<0$ for all $\xi \in W^{*} \backslash\{0\}$.

In fact, $\operatorname{det} \mathfrak{T}_{\xi} \neq 0$ whenever $\xi \in W^{*} \backslash\{0\}$, for otherwise there would exist $v \in Q \backslash\{0\}$ such that the injective operator $\gamma(\cdot, v)$ sends $P$ into the subspace Ker $\xi \subset W$ of dimension $n-1$. Our claim now follows since $W^{*} \backslash\{0\}$ is connected.

Lemma 16.2. Let totally real oriented planes $P$ and $Q$ in a complex plane $V$ have a negative transverse intersection at 0 , and let $\Gamma$ be the canonically-oriented unit circle $\{x \in P:\langle x, x\rangle=1\}$ for a fixed Euclidean inner product $\langle$,$\rangle in P$. The oriented cylinder $\mathbf{R} \times \Gamma$ then admits a totally real embedding $f$ in $V$ such that $f(t, x)=|t| x$ if $(t, x) \in(-\infty,-r] \times \Gamma$ and $f(t, x)=t A x$ if $(t, x) \in[r, \infty) \times \Gamma$, for some positive real number $r$ and some orientation-preserving real-linear isomorphism $A: P \rightarrow Q$.

Any $f$ as above and $x \in \Gamma$ give rise to the curve $[-r, r] \ni t \mapsto \mathfrak{L}^{+}\left(W_{t, x}\right)$ in the circle $\mathrm{S}\left(V^{\wedge 2}\right)$, where $\mathfrak{L}^{+}$and $\mathrm{S}\left(V^{\wedge 2}\right)$ are as in (3.1), while $W_{t, x}$ denotes the oriented plane tangent to $f(\mathbf{R} \times \Gamma)$ at $f(t, x)$. The curves arising in this way from all such $f$ represent all fixed-endpoint homotopy classes of curves joining $\mathfrak{L}^{+}(P)$ to $-\mathfrak{L}^{+}(Q)$ in $\mathrm{S}\left(V^{\wedge 2}\right)$. 
We now proceed to discuss some consequences of Lemma 16.2 (a proof of which is given in Section 17).

Suppose that a totally real immersed surface $\Sigma$ in an almost complex surface $M$ has only one self-intersection, in the form of a transverse double point at some $y \in M$, and either $\Sigma$ is nonorientable, or it is orientable and the self-intersection is negative (as defined above). Then a totally real surface $\Sigma^{\prime}$ smoothly embedded in $M$ and diffeomorphic to $\Sigma \# K^{2}$, where $K^{2}$ is the Klein bottle, can be obtained from $\Sigma$ by replacing the disjoint union of disk-like neighborhoods of $y$ in the two branches of $\Sigma$ through $y$ with a cylinder contained in an open subset of $M$ diffeomorphic to $\mathbf{R}^{4}$.

To see this, let us identify a neighborhood of $y$ in $M$ with a neighborhood $U$ of 0 in a complex plane $V$ in such a way that $y=0$, the almost complex structure $J$ of $M$ coincides at $y=0$ with the standard complex structure of $V$, and $\Sigma \cap U=(P \cup Q) \cap U$ for some oriented totally real planes $P, Q$ through 0 in $V$ having a negative intersection at 0 . If $\Sigma$ is orientable, we fix an orientation of $\Sigma$ and require, in addition, that the orientations of $P$ and $Q$ agree with those of the two branches of $\Sigma$ through $y$. For $f$ and $r$ chosen as in Lemma 16.2, the image $Y$ of the Gauss mapping $\mathrm{G}_{f}$ (see (10.1) ) is a compact subset of $\mathrm{Gr}_{2}(V)$, as $\mathrm{G}_{f}((-\infty,-r] \times \Gamma)=\{P\}$ and $\mathrm{G}_{f}([r, \infty) \times \Gamma)=\{Q\}$. Next, we choose an open set $U^{\prime} \subset U$ diffeomorphic to $\mathbf{R}^{4}$ such that $J(x) \in \operatorname{Hom}_{\mathbf{R}}(V, V)$ lies in $\Omega$ whenever $x \in U^{\prime}$, for $\Omega$ obtained by applying Lemma 10.3 to our $Y$ and $J=J(0)$. We also select $\varepsilon \in(0, \infty)$ with $\varepsilon f([-r, r] \times \Gamma) \subset U^{\prime}$. Our claim is now obvious, the embedded cylinder being $\varepsilon f((-r, r) \times \Gamma)$.

In Section 36 we give another application of Lemma 16.2 which also uses the assertion about homotopy classes.

\section{Proof of Lemma 16.2}

Totally real planes through 0 in $V$ form an open set $\operatorname{TR}(V)$ in the 4-manifold $\operatorname{Gr}_{2}(V)$. The circle $\mathbf{R P}^{1}=\mathrm{U}(1) / \mathbf{Z}_{2}$ acts on $\mathrm{TR}(V)$ freely by $( \pm z, Q) \mapsto z Q$ for $z \in \mathrm{U}(1) \subset \mathbf{C}$ and $Q \in \mathrm{TR}(V)$. The quotient $\mathrm{TR}(V) / \mathbf{R P}^{1}$ can be diffeomorphically identified, as described next, with the 3 -manifold $N$ of all plane circles in the unit sphere $S^{2} \subset \mathbf{R}^{3}$ (that is, circles obtained by intersecting $S^{2}$ with planes which need not pass through 0 ). The corresponding principal $\mathbf{R} \mathbf{P}^{1}$-bundle projection $\pi: \operatorname{TR}(V) \rightarrow N$ sends $Q \in \mathrm{TR}(V)$ to the image $\pi(Q)$ of $Q \backslash\{0\}$ under the standard projection $V \backslash\{0\} \rightarrow \mathrm{P}(V) \approx \mathrm{CP}^{1} \approx S^{2}$.

In fact, each such image $\pi(Q)$ is a circle in the Riemann sphere $S^{2} \approx \mathbf{C} \cup\{\infty\}$, as one sees by choosing an isomorphic identification $V=\mathbf{C}^{2}$ under which $Q=\mathbf{R}^{2}$ (and so $\pi(Q)=\mathbf{R} \cup\{\infty\} \subset \mathbf{C} \cup\{\infty\}$ ). Furthermore, if $Q, Q^{\prime} \in \operatorname{TR}(V)$ and $\pi(Q)=\pi\left(Q^{\prime}\right)$, then $z u, a v, c(u+v) \in Q^{\prime}$ for any fixed basis $u, v$ of $Q$ and some $z, a, c \in \mathbf{C} \backslash\{0\}$, so that, writing $c(u+v)$ as a real combination of $z u$ and av (which span $Q^{\prime}$ over $\mathbf{R}$ ), we get $a / z \in \mathbf{R}$, and hence $Q^{\prime}=z Q$. Finally, $\pi: \operatorname{TR}(V) \rightarrow N$ has constant rank due to its equivariance relative to the transitive actions of the linear group $\operatorname{GL}(V) \approx \operatorname{GL}(2, \mathbf{C})$ on $\mathrm{TR}(V)$ and of the complex automorphism group $\operatorname{Aut}\left(S^{2}\right)$ on $N$, for the standard surjective homomorphism $\operatorname{GL}(V) \rightarrow \operatorname{Aut}\left(S^{2}\right)=\operatorname{PGL}(V)$.

Note that $N=\tilde{N} / \mathbf{Z}_{2}$, where $\widetilde{N}$ is the manifold of all oriented plane circles in $S^{2}$ (diffeomorphic to $S^{2} \times(0, \pi)$ via the center-radius parametrization of oriented 
circles in the oriented 2-sphere), and the fixed-point free involution generating the $\mathbf{Z}_{2}$ action reverses the circle orientations.

We begin by proving our assertion in the special case where the circles $\pi(P), \pi(Q)$ in $S^{2}$ are disjoint. We then choose a smoothly embedded curve segment joining $\pi(P)$ to $\pi(Q)$ in $N$, consisting of pairwise disjoint circles, and lift it: first to the bundle space $\operatorname{TR}(V)$ over $N$, and from there to the bundle over $\operatorname{TR}(V)$ formed by the manifold of all $\mathbf{C}$-bases of $V$, which we identify with $\operatorname{GL}(V)$ using a fixed basis of $P$. This yields a $C^{\infty}$ embedding $[-r, r] \ni t \mapsto B_{t} \in \mathrm{GL}(V)$, with any fixed $r \in(0, \infty)$, such that $B_{-r}=\mathrm{Id}, B_{r} P=Q$, and the circles $\pi\left(B_{t} P\right)$ are pairwise disjoint for all $t \in[-r, r]$.

Next, we replace the curve $t \mapsto B_{t}$ by a composite in which the original curve is preceded by a $C^{\infty}$ function $\mathbf{R} \rightarrow[-r, r]$ equal to $-r$ on $(-\infty,-r]$, to $r$ on $[r, \infty)$, and having a positive derivative everywhere in $(-r, r)$. The resulting new curve, still written as $t \mapsto B_{t}$ (but now defined on $\mathbf{R}$ ), leads to the required totally real embedding $f: \mathbf{R} \times \Gamma \rightarrow V$, given by $f(t, x)=e^{\varphi(t)} B_{t} x$ for a $C^{\infty}$ immersion $\varphi: \mathbf{R} \rightarrow \mathbf{C}$ with $\varphi(t)=\log |t|$ whenever $|t| \geq r$. The choice of $\varphi$ on $(-r, r)$ is described below.

We denote by $I$ the complex-linear operator $V \rightarrow V$ whose restriction to the oriented Euclidean plane $P$ is the positive rotation by the angle $\pi / 2$. If $f$ corresponds as above to any given $C^{\infty}$ immersion $\varphi: \mathbf{R} \rightarrow \mathbf{C}$, then

$$
d f_{(t, x)}(1,0)=e^{\varphi}(\dot{\varphi} B+\dot{B}) x \text { and } d f_{(t, x)}(0, I x)=e^{\varphi} B I x
$$

for the basis $(1,0),(0, I x)$ of $T_{(t, x)}(\mathbf{R} \times \Gamma)$. (We omit $t$ in our notation, and set ()$^{\circ}=d / d t$.) Thus, $f$ is a totally real immersion if and only if, for every $(t, x) \in \mathbf{R} \times \Gamma$, the $\left(e^{\varphi} B\right.$-images of the $)$ vectors $\left(\dot{\varphi}+B^{-1} \dot{B}\right) x$ and $I x$ are linearly independent over $\mathbf{C}$. Since $P$ is totally real, we may identify $V^{\wedge 2}$ with $\mathbf{C}$ in such a way that $x \wedge I x=1$ for some $x \in \Gamma$, and so the linear independence condition will follow if $\dot{\varphi} \neq-s B^{-1} \dot{B} x \wedge I x$ for all $(s, t, x) \in[0,1] \times[-r, r] \times \Gamma$. (Note that $x \wedge I x=1$ for every $x \in \Gamma$, as each pair $x, I x$ is a positive orthonormal basis of P.) The condition holds if $|t| \geq r$, since we then have $\dot{\varphi} \neq 0=\dot{B}$.

Such an immersion $\varphi$, with $\varphi(t)=\log |t|$ whenever $|t| \geq r$, exists and may be chosen so that $\dot{\varphi}:[-r, r] \rightarrow \mathbf{C} \backslash\{0\}$ belongs to any prescribed fixed-endpoint homotopy class of curves joining $-1 / r$ to $1 / r$ in $\mathbf{C} \backslash\{0\}$. In fact, as $\dot{B}=0$ whenever $|t| \geq r$, there exist constants $\kappa>0$ and $\delta \in(0, r)$ with $\left|B^{-1} \dot{B} x \wedge I x\right| \leq \kappa$ for all $(t, x) \in[-r, r] \times \Gamma$, and $\left|B^{-1} \dot{B} x \wedge I x\right|<1 / r$ if, in addition, $|t| \geq \delta$. We now select $\varphi$ with $\varphi(-t)=\varphi(t)$ and $|\dot{\varphi}(t)|>1 / r$ whenever $|t| \in[\delta, r)$, such that one also has $|\dot{\varphi}|=\mu$ on $[-\delta, \delta]$ for a constant $\mu>\kappa$. As a result, $f$ is a totally real immersion, and it is also injective due to pairwise disjointness of the circles $\pi\left(B_{t} P\right)$ for $t \in[-r, r]$. A curve $\varphi:[-\delta, \delta] \rightarrow \mathbf{C}$ with the constant speed $\mu$ and a fixed length can clearly be chosen so that, before returning at time $t=\delta$ to the initial point $\varphi(-\delta)$ with the velocity $-\dot{\varphi}(-\delta)$, it traverses any prescribed number $q$ of times, either clockwise or counterclockwise, some circle of radius depending on $q$. This produces the required homotopy effect: using the definition of $\mathfrak{L}^{+}$(see (3.1)), our identification $V^{\wedge 2}=\mathbf{C}$ with $x \wedge I x=1$ for all $x \in \Gamma$, and (17.1), we see that the homotopy classes in question correspond to those of the curves $t \mapsto e^{\varphi}(\dot{\varphi} B+s \dot{B}) x \wedge e^{\varphi} B I x$ with $s=1$, joining $-r$ to $r$ in $\mathbf{C} \backslash\{0\}$. However, we may replace $s=1$ by $s=0$, since our choice of $\varphi$ turns $s \in[0,1]$ into a homotopy parameter. The resulting homotopy class of $t \mapsto e^{2 \varphi} \dot{\varphi} \operatorname{det} B$ equals 
that of $t \mapsto \dot{\varphi} \operatorname{det} B$ (we deform the factor $e^{2 \varphi}$ to a constant in $\mathbf{C} \backslash\{0\}$ by first deforming $\varphi$ in $\mathbf{C}$ ). As the curve $t \mapsto B_{t}$ does not depend on $\varphi$, our claim about homotopy classes follows.

Also, $B_{r}: P \rightarrow Q$ is an orientation-preserving isomorphism. Namely, using the original orientation of $P$, we now orient the planes $Q_{t}=B_{t} P, t \in(-r, r]$, by requiring that $B_{t}: P \rightarrow Q_{t}$ be orientation-preserving. This might, possibly, result in re-orienting $Q=Q_{r}$, and we will prove that is does not, by showing that at least one newly-oriented $Q_{t}, t \in(-r, r]$, has a negative intersection with $P$ at 0 . (The words 'at least one' then may be replaced with 'every' by reasons of continuity.) Let us fix $t \in(-r, r]$. The circles $\pi(P)$ and $\pi\left(Q_{t}\right)$ are disjoint, so that $\gamma: P \times Q_{t} \rightarrow V^{\wedge 2}$ given by $\gamma(u, v)=u \wedge v$ satisfies the hypotheses of Lemma 16.1 for $n=2$. Identifying $V^{\wedge 2}$ with $\mathbf{C}$ as before, we may apply the conclusion of Lemma 16.1 to the bases $u_{j}, v_{k}$ with $u_{1}=x, u_{2}=I x$ (where $x \in \Gamma$ ), and $v_{j}=B_{t} u_{j}$ for $j=1,2$, along with $\xi=\operatorname{Re}$ and $\xi=\operatorname{Im}$. Taking the limit as $t \rightarrow r$, we have $B_{t} \rightarrow \mathrm{Id}$, and so $\operatorname{det} \mathfrak{T}_{\xi}>0$ for $\xi=\mathrm{Im}$ and $t>r$ close to $r$, since $\operatorname{det} \mathfrak{T}_{\xi} \rightarrow 1$ as $t \rightarrow r$ when $\xi=$ Re. (In fact, the limit, as $t \rightarrow r$, of the complex $2 \times 2$ matrix with the entries $\gamma\left(u_{j}, v_{k}\right)$ has the rows [0 1$]$ and $\left[\begin{array}{ll}-1 & 0\end{array}\right]$.) Writing $\left[\begin{array}{ll}v_{1} & v_{2}\end{array}\right]=\left[\begin{array}{ll}u_{1} & u_{2}\end{array}\right] \mathfrak{B}$, where $\mathfrak{B}$ is a complex $2 \times 2$ transition matrix depending on $t$, we get the transition formula

$$
\left[\begin{array}{llll}
u_{1} & u_{2} & v_{1} & v_{2}
\end{array}\right]=\left[\begin{array}{llll}
u_{1} & u_{2} & i u_{1} & i u_{2}
\end{array}\right]\left[\begin{array}{cc}
\operatorname{Id} & \operatorname{Re} \mathfrak{B} \\
0 & \operatorname{Im} \mathfrak{B}
\end{array}\right]
$$

with a real $4 \times 4$ matrix composed of four $2 \times 2$ blocks. As one easily verifies, $\operatorname{det} \mathfrak{T}_{\operatorname{Im}}=\operatorname{det} \operatorname{Im} \mathfrak{B}$, so that $\operatorname{det} \operatorname{Im} \mathfrak{B}>0$. Negativity of the intersection follows: the basis $u_{1}, u_{2}, i u_{1}, i u_{2}$ represents the opposite of the standard orientation of $V$; cf. Section 5 .

Using $\varphi$ as above, the corresponding $f$, and $A=B_{r}$, we thus get our claim under the additional assumption that $\pi(P) \cap \pi(Q)=\varnothing$. The general assertion, for an arbitrary pair $P, Q$, can now be derived from a reduction argument. Namely, to prove it for the given $P, Q$, we just need to establish it for the pair $R, Q$ with some oriented Euclidean plane $R$ satisfying the same hypotheses as $P$ and such that there exist a totally real embedding $h: P \rightarrow V$, an orientation-preserving linear isometry $\Psi: P \rightarrow R$, and constants $c, r^{\prime}$, for which $r^{\prime}>c>r$ and $h(P) \cap Q=\{0\}$, as well as

(a) $h(y)=\Psi(y)$ whenever $y \in P$ and $|y| \leq c$,

(b) $h(y)=y$ whenever $y \in P$ and $|y| \geq r^{\prime}$,

where $r$, along with some $A$ and $f: \mathbf{R} \times \Gamma \rightarrow V$, satisfies the assertion of the lemma for $R, Q$ instead of $P, Q$. In fact, given such $R, h, \Psi, c, r^{\prime}$, we define the analogues of $r, A, f$ for $P$ and $Q$ to be $r^{\prime}, A \Psi$, and the mapping $f^{\prime}$ given by $f^{\prime}(t, x)=h(|t| x)$ if $t \leq-r$ and $f^{\prime}(t, x)=\varepsilon f(t / \varepsilon, \Psi x)$ if $t \geq-c$, for suitable $\varepsilon>0$. Both formulae for $f^{\prime}$ clearly define $V$-valued totally real embeddings of appropriate domains. They also agree, yielding $|t| \Psi x$, when $-c \leq t \leq-r$ (and $x$ lies in the unit circle $\Gamma^{\prime}=\Psi^{-1}(\Gamma) \subset P$ ); thus, the only property of $f^{\prime}$ that we need to verify is injectivity, which amounts to $f^{\prime}\left((-\infty,-r] \times \Gamma^{\prime}\right) \cap f^{\prime}\left((-r, \infty) \times \Gamma^{\prime}\right)=\varnothing$. However, if $\varepsilon \in(0,1)$, the definition of $f^{\prime}$ implies that $f^{\prime}\left((-r, \infty) \times \Gamma^{\prime}\right)$ is contained in the union of three sets: the radius $r$ open disk $D$ about 0 in $R$, the compact set $\varepsilon S$, where $S=f([-r, r] \times \Gamma)$, and $Q$. None of the three sets intersects $Y=f^{\prime}\left((-\infty,-r] \times \Gamma^{\prime}\right)$ if $\varepsilon$ is chosen so that $\varepsilon S \subset U$ for a neighborhood $U$ of 
0 in $V$ with $U \cap Y=\varnothing$. In fact, $D \cap Y=\varnothing$ since $D$ and $Y$ are the $h$-images of $\Psi^{-1}(D)$ and $P \backslash \Psi^{-1}(D)$, while $Q \cap Y=\varnothing$ as $Y \subset h(P \backslash\{0\})$ and $h(P) \cap Q=\{0\}$. Finally, such $f^{\prime}$ realize all the homotopy classes required in the lemma, since we assume here that the same can be achieved by varying the choices of $f$ for the pair $R, Q$.

We apply the above reduction argument twice. In both cases, using arbitrary $c, r^{\prime}$ with $r^{\prime}>c>r$, we obtain $R, h, \Psi$ by exhibiting a 3 -dimensional real vector subspace $W \subset V$ containing both $P$ and a plane $R$ with $P \cap R \cap L=R \cap W \cap Q=$ $\{0\}$, where $L$ is (always) the unique complex line through 0 in $V$ contained in $W$. Note that $\operatorname{dim}(W \cap Q)=1$, as $Q$ intersects $P$ trivially and so cannot be contained in $W$. Also, if $\mathrm{pr}: W \rightarrow \Pi$ denotes the quotient projection onto the plane $\Pi=W /(P \cap R)$, it is clear that $\operatorname{pr}(L)=\Pi$, while $\Lambda_{1}=\operatorname{pr}(P), \Lambda_{2}=\operatorname{pr}(R)$ and $\Lambda_{3}=\operatorname{pr}(W \cap Q)$ are three distinct lines in $\Pi$. We may thus choose a vector $v \in L \backslash\{0\}$ such that $\operatorname{pr} v \notin \Lambda_{1} \cup \Lambda_{2} \cup \Lambda_{3}$, while, for $\Lambda_{4}=\operatorname{pr}(\mathbf{R} v)$ and some Euclidean norm in $\Pi$, an arc of the unit circle around 0 in $\Pi$ intersects each of the four lines $\Lambda_{j}$ just once, in this order: $\Lambda_{1}, \ldots, \Lambda_{4}$. Let us refer to the direction of $v$ in $W$ as vertical. For every $C^{\infty}$ function $\varphi: P \rightarrow \mathbf{R}$, the embedding $h: P \rightarrow V$ given by $h(y)=y+\varphi(y) v$ is totally real, since so is the surface $h(P)$. (As $L$ contains the vertical vector $v$, it cannot be a tangent plane of a graph.) To get (a), (b) and $h(P) \cap Q=\{0\}$, we now set $\varphi=\varphi_{1} \varphi_{2}$, where $\varphi_{1}$ is a $C^{\infty}$ function $P \rightarrow[0,1]$ with $\varphi_{1}(y)=1$ whenever $|y| \leq c$ and $\varphi_{1}(y)=0$ whenever $|y| \geq r^{\prime}$, while $\varphi_{2}$ is a linear functional on $P$ having the graph $R$. (Such $\varphi_{2}$ exists since the vertical vector $v$ does not lie in $R$.) The condition $h(P) \cap Q=\{0\}$ follows as $\operatorname{pr}(h(P)) \cap \operatorname{pr}(W \cap Q)=\{0\}$ in $\Pi$ and $\operatorname{pr}: W \cap Q \rightarrow \Pi$ is injective, while the orientation and inner product in $R$ are chosen so as to make $\Psi: P \rightarrow R$, with $\Psi y=y+\varphi_{2}(y)$, an orientation-preserving linear isometry, and the intersection of $R$ and $Q$ then is negative due to our choice of $v$.

Using the fact that our claim holds when $\pi(P) \cap \pi(Q)=\varnothing$, we now establish it under the weaker additional assumption $\pi(P) \neq \pi(Q)$. Specifically, $W$ and $R$ required above are selected by fixing $R \in \mathrm{TR}(V)$ for which $\pi(R) \cap \pi(Q)=\varnothing$ and $\pi(P) \cap \pi(R)$ has two elements. We may also assume that $\operatorname{dim}(P \cap R)=1$ (which is achieved by replacing $R$ with $z R$ for a suitable complex number $z \neq 0$ ) and then we set $W=\operatorname{Span}_{\mathbf{R}}(P \cup R)$. Thus, $P \cap R \cap L=\{0\}$ for $L \subset W$ as above: one element of $\pi(P) \cap \pi(R)$ is $\operatorname{Span}_{\mathbf{C}}(P \cap R)$, and so $L$ must be its other element, spanned over $\mathbf{R}$ by a line in $P$ and a line in $R$ different from $P \cap R$. In view of the reduction argument, our assertion, valid for $R$ and $Q$ (since $\pi(R) \cap \pi(Q)=\varnothing$ ), hold for $P, Q$ as well.

Similarly, using our conclusion for arbitrary $P, Q$ with $\pi(P) \neq \pi(Q)$, we now prove it in the remaining case $\pi(P)=\pi(Q)$. Namely, we choose $W$ and $R$ to be any real 3 -space in $V$ containing $P$ and, respectively, any plane in $W$ with $P \cap R \cap L=\{0\}$, for the complex line $L \subset W$. Thus, $\pi(R) \neq \pi(P)=\pi(Q)$, so that our claim is true for $R, Q$ (see the last paragraph), and, consequently, it holds for $P, Q$ as well.

\section{Connected sums}

The main result of this section is Theorem 18.1 which, essentially, establishes the existence of a connected-sum operation in the category of totally real surfaces immersed in a fixed almost complex surface $M$. The totally real immersed cylinder 
joining the two surfaces is of the type known as Whitney's umbrella; it has a unique self-intersection (which is transverse and negative, in the sense of Section 16). Since there always exist arbitrarily many pairwise disjoint totally real tori embedded in $M$ (Corollary 10.2), a self-intersection cannot, in general, be avoided. Otherwise, connected sums of such tori would constitute totally real closed orientable surfaces of all genera $s \geq 2$, embedded in any given $M$, in patent contradiction to Corollary 2.10.

Theorem 18.1. Let $\Sigma^{ \pm}$be two disjoint totally real surfaces embedded in an almost complex surface $M$, both endowed with the subset topology and closed as subsets of M. Also, let $x^{ \pm} \in \Sigma^{ \pm}$. Then, for any sufficiently small closed 2-disks $D^{ \pm}$diffeomorphically embedded in $\Sigma^{ \pm}$and containing $x^{ \pm}$as interior points, there exist a totally real surface $\Sigma$ immersed in $M$ and an immersion $h:[-1,1] \times S^{1} \rightarrow M$ such that

(i) $\Sigma$ is the disjoint union of $\Sigma^{+} \backslash D^{+}, \Sigma^{-} \backslash D^{-}$and the image of $h$,

(ii) $h$ sends the circles $\{ \pm 1\} \times S^{1}$ onto $\partial D^{ \pm}$,

(iii) $\Sigma$ has just one self-intersection: a double point of $h$ in $(-1,1) \times S^{1}$,

(iv) the self-intersection of $\Sigma$ is transverse and negative; cf. Section 16.

(v) the union of $D^{+} \cup D^{-}$and the image of $h$ is contained in an open subset of $M$ diffeomorphic to $\mathbf{R}^{4}$.

We prove Theorem 18.1 in Section 19, First, we need three lemmas, in which ()$^{*}=d / d t,()^{\prime}=d / d s,()_{t}=\partial / \partial t$ and ()$_{s}=\partial / \partial s$ stand for ordinary or partial derivatives with respect to real variables $t$ and $s$.

Lemma 18.2. If $c, \varepsilon \in(0, \infty)$, while $t \mapsto \varphi(t)$ and $s \mapsto \psi(s)$ are $C^{\infty}$ functions of $t \in(-c, c)$ and $s \in(-\varepsilon, \varepsilon)$, valued in a finite-dimensional vector space $V$, then the following two conditions are equivalent:

(a) $\varphi(t)=F_{s}(t, 0)$ and $\psi(s)=F_{t}(0, s)$ for all $(t, s) \in(-c, c) \times(-\varepsilon, \varepsilon)$ and some $C^{\infty}$ function $F:(-c, c) \times(-\varepsilon, \varepsilon) \rightarrow V$ having the property that $F(t, 0)=F(0, s)=0$ whenever $t \in(-c, c)$ and $s \in(-\varepsilon, \varepsilon)$,

(b) $\varphi(0)=\psi(0)=0$ and $\dot{\varphi}(0)=\psi^{\prime}(0)$.

In fact, (a) implies (b): $\dot{\varphi}(0)=F_{s t}(0,0)=F_{t s}(0,0)=\psi^{\prime}(0)$. Conversely, assuming (b), we may set $F(t, s)=t s[\alpha(t)+\beta(s)-\dot{\varphi}(0)]$ for $C^{\infty}$ functions $\alpha, \beta$ with $\varphi(t)=t \alpha(t)$ and $\psi(s)=s \beta(s)$ (so that $\alpha(0)=\beta(0)=\dot{\varphi}(0)$ ).

Lemma 18.3. Given an almost complex surface $M$, a point $y \in M$, and a vector $u \in T_{y} M \backslash\{0\}$, there exists an $M$-valued $C^{\infty}$ embedding $f$ of a neighborhood of 0 in $\mathbf{C}$ such that, for all real $t, s$ near 0 , the differentials $d f_{t}$ and $d f_{i s}$ are complex-linear, while $f(0)=y$ and $d f_{0}$ sends the vectors $1, i \in \mathbf{C}=T_{0} \mathbf{C}$ to $u$ and $J u$.

Proof. Let us fix a complex plane $V$ and a diffeomorphic identification of a neighborhood of $y$ in $M$ with a neighborhood of 0 in $V$, under which $y=0$ and the almost complex structure $J$ of $M$ equals, at 0 , the standard structure of $V$ (represented by the symbol $i$ ). Thus, $u, i u \in V=T_{y} M$. For real $t, s$ near 0 we now set $f(t+i s)=(t+i s) u+t^{2} v+F(t, s)$, where $v \in V$ and $F$ is a $C^{\infty}$ function $(-c, c) \times(-\varepsilon, \varepsilon) \rightarrow V$, with sufficiently small $c, \varepsilon$, such that $F(t, s)=0$ whenever $t s=0$. Clearly, $d f_{t}$ (or, $d f_{i s}$ ) sends the real basis $\{1, i\}$ of $\mathbf{C}=T_{t} \mathbf{C}$ (or, $\mathbf{C}=T_{i s} \mathbf{C}$ ) to $\left\{u+2 t v, i u+F_{s}(t, 0)\right\}$ (or, respectively, to $\left\{u+F_{t}(0, s), i u\right\}$ ). 
Thus, to ensure complex-linearity of all $d f_{t}$ and $d f_{i s}$, we need to choose $F$ and $v$ so that $F_{s}(t, 0)=\varphi(t)$ and $F_{t}(0, s)=\psi(s)$ for all $t, s$, where $\varphi(t)=J u+2 t J v-i u$ and $\psi(s)=-u-J i u$. Note that $u, i u, v$ are vectors (i.e., constant vector fields on $V$ ), while here $J$ denotes the real-linear operator $J_{x}: V \rightarrow V$, depending on $x=f(t)$ or, respectively, $x=f(i s)$. Finally, $F$ with $F_{s}(t, 0)=\varphi(t)$ and $F_{t}(0, s)=\psi(s)$ exists, by Lemma 18.2. if and only if the sum $d_{u}(J u)+d_{i u}(J i u)$ of directional derivatives at 0 equals $-2 i v$. With this choice of $v$, our assertion follows.

Let $\Sigma$ be a totally real surface embedded in an almost complex surface $M$ and contained in a fixed 3 -dimensional real submanifold $N$ of $M$. The characteristic foliation of $\Sigma$ in $N$ is the 1-dimensional foliation on $\Sigma$ tangent, at every point $x \in \Sigma$, to the characteristic direction $L_{x} \cap T_{x} \Sigma$, for the unique complex line $L_{x}$ in $T_{x} M$ with $0 \in L_{x} \subset T_{x} N$. By a characteristic curve of $\Sigma$ in $N$ we mean any leaf of its characteristic foliation.

Remark 18.4. Given $M, N$ and $\Sigma$ as above, let $K$ be another real surface embedded in $M$ with $K \subset N$. If $y \in K \cap \Sigma$, while $T_{y} \Sigma \cap T_{y} K$ is one-dimensional and different from the characteristic direction, at $y$, of $\Sigma$ in $N$, then $K$ is totally real at $y$, in the sense that $y$ is not a complex point of $K$. (In fact, if $T_{y} K$ were a complex line, the characteristic direction of $\Sigma$ at $y$ would, by definition, be $T_{y} \Sigma \cap T_{y} K$.)

Lemma 18.5. Let $M, \Sigma^{ \pm}, x^{ \pm}$satisfy the hypotheses of Theorem 18.1, and let $U_{\varepsilon}$, with $\varepsilon \in(0, \infty)$, be the set of $(z, w) \in \mathbf{C}^{2}$ such that $|\operatorname{Re} z|-1,|\operatorname{Im} z|,|\operatorname{Re} w|$ and $|\operatorname{Im} w|$ are all less than $\varepsilon$. If the sets $N \subset U_{\varepsilon}$ and $\Sigma_{t} \subset N$ are given by $N=\left\{(z, w) \in U_{\varepsilon}: \operatorname{Im} w=0\right\}$ and $\Sigma_{t}=\left\{(z, w) \in U_{\varepsilon}: \operatorname{Re} z=t\right.$ and $\left.\operatorname{Im} w=0\right\}$, for any fixed $t \in(-1-\varepsilon, 1+\varepsilon)$, then the following conclusions hold for some $\varepsilon>0$ and some $C^{\infty}$-diffeomorphic identification of $U_{\varepsilon}$ with an open set in $M$ :

(a) $x^{ \pm}=( \pm 1,0) \in U_{\varepsilon} \subset \mathbf{C}^{2}$ and $\Sigma^{ \pm} \cap U_{\varepsilon}=\Sigma_{ \pm 1}$.

(b) Each $\Sigma_{t}$ is a totally real surface embedded in $M$, and its characteristic foliation in the ambient 3-manifold $N$ consists of line segments parallel to the $\operatorname{Im} z$ coordinate direction.

(c) The surface $Y=\{(z, w) \in N: \operatorname{Im} z=0\}$ is totally real in $M$, and its characteristic foliation relative to $N$ consists of line segments parallel to the $\operatorname{Re} z$ coordinate direction.

(d) The almost complex structure $J$ of $M$ coincides with the standard complex structure of the open set $U_{\varepsilon} \subset \mathbf{C}^{2}$ at all points $(z, w) \in U_{\varepsilon}$ such that (i) $\operatorname{Im} z=w=0$ or (ii) $\operatorname{Re} z=w=0$.

(e) The coordinate vector fields $u$ and $v$ corresponding to the $\operatorname{Re} z$ and $\operatorname{Re} w$ directions are $J$-linearly independent at every point of $U_{\varepsilon}$.

Proof. Let us fix a point $y \in M \backslash\left(\Sigma^{+} \cup \Sigma^{-}\right)$and a nonzero vector $u \in T_{y} M$. We then choose an embedding $f$ with the properties listed in Lemma 18.3 and three coordinate systems, on neighborhoods of $x^{+}, x^{-}$and $y$, under which $x^{+}, x^{-}$and $y$ all have zero coordinates, while some neighborhoods of $x^{ \pm}$in $\Sigma^{ \pm}$correspond to neighborhoods of $(0,0,0,0)$ in the submanifold $\mathbf{R}^{2} \times\{(0,0)\}$ of $\mathbf{R}^{4}$, and $f$ appears, in the coordinates chosen at $y$, as the mapping $\mathbf{C} \ni z \mapsto(z, 0) \in \mathbf{C}^{2}=\mathbf{R}^{4}$ restricted to a neighborhood of $0 \in \mathbf{C}$.

Let exp now be the exponential mapping of a Riemannian metric on $M$ chosen so that, on some neighborhoods of $x^{+}, x^{-}$and $y$, the metric is flat and the above 
coordinate mappings are isometries onto neighborhoods of $(0,0,0,0)$ in $\mathbf{R}^{4}$ with the Euclidean metric. (Such a metric on $M$ can easily be obtained using a finite partition of unity.)

A $C^{\infty}$ diffeomorphism $H: U_{\varepsilon} \rightarrow H\left(U_{\varepsilon}\right)$ onto an open set $H\left(U_{\varepsilon}\right) \subset M$, providing the required diffeomorphic identification, will be constructed in four successive steps, each of which consists in defining $H$ just on some subset of $U_{\varepsilon}$ (and making $\varepsilon$ smaller, if necessary). The four subsets are: the interval $I_{\varepsilon}$ of all $(t, 0) \in \mathbf{C}^{2}$ such that $t \in \mathbf{R}$ and $|t|<1+\varepsilon$, the rectangle $R_{\varepsilon}$ formed by all $(z, 0) \in \mathbf{C}^{2}$ with $|\operatorname{Re} z|<1+\varepsilon$ and $|\operatorname{Im} z|<\varepsilon$, the 3-manifold $N_{\varepsilon}=\left\{(z, w) \in U_{\varepsilon}: \operatorname{Im} w=0\right\}$ (which is nothing other than the set $N$ appearing in the lemma), and $U_{\varepsilon}$ itself. Thus, $I_{\varepsilon} \subset R_{\varepsilon} \subset N_{\varepsilon} \subset U_{\varepsilon}$.

We begin by restricting $f$ chosen earlier in this proof to a small neighborhood of 0 in $\mathbf{R} \subset \mathbf{C}$ and then extending this restriction to a $C^{\infty}$ embedding $t \mapsto x(t) \in M$ defined for real $t$ with $|t|<1+\varepsilon$, such that $x( \pm 1)=x^{ \pm}$and $J \dot{x}( \pm 1)$ is, for either sign \pm , tangent to $\Sigma^{ \pm}$at $x^{ \pm}$. (To realize the latter condition, it suffices to prescribe the velocity $\dot{x}( \pm 1)$ as a vector in $J\left(T_{x( \pm 1)} \Sigma^{ \pm}\right)$.) We now choose $H: I_{\varepsilon} \rightarrow M$ by setting $H(t, 0)=x(t)$.

Making $\varepsilon$ smaller, we extend $H$ from the interval $I_{\varepsilon}$ to the rectangle $R_{\varepsilon}$ by setting $H(z, 0)=\exp _{x(t)} s J \dot{x}(t)$, where $t, s \in \mathbf{R}$ and $z=t+i s$. Note that $H$ is injective on $I_{\varepsilon}$, and so is the differential of $H: R_{\varepsilon} \rightarrow M$ at each point of $I_{\varepsilon}$. Hence $H: R_{\varepsilon} \rightarrow M$ is, for small $\varepsilon>0$, an embedding.

Next, we choose a $C^{\infty}$ vector field $I_{\varepsilon} \ni(t, 0) \mapsto v(t) \in T_{x(t)} M$, nowhere tangent to the embedded surface $H\left(R_{\varepsilon}\right)$, and such that $v( \pm 1)$ is tangent to $\Sigma^{ \pm}$at $x^{ \pm}$. Its $C^{\infty}$ extension $R_{\varepsilon} \ni(z, 0) \mapsto \tilde{v}(z, 0) \in T_{H(z, 0)} M$, obtained using parallel transports along the curves $s \mapsto H(t+i s, 0)$, for $t, s$ as before, gives rise to the mapping $\tilde{H}: N \rightarrow M$ with $\tilde{H}(z, r)=\exp _{H(z, 0)} r \tilde{v}(z, 0)$, where $N=N_{\varepsilon}$. As in the last paragraph, $\tilde{H}$ is an embedding for sufficiently small $\varepsilon>0$. Also, for small $\varepsilon$, the $\tilde{H}$-images of the surfaces $Y \subset N$ and $\Sigma_{t} \subset N$ with $|t|<1+\varepsilon$, defined in the lemma, are all totally real in $M$, while $\tilde{H}\left(I_{\varepsilon}\right)$ (or $\{\tilde{H}(i s, 0): s \in(-\varepsilon, \varepsilon)\}$ ) is a characteristic curve of $\tilde{H}(Y)$ (or, respectively, of $\tilde{H}\left(\Sigma_{0}\right)$ ) in $\tilde{H}(N)$, and the $\tilde{H}$ image of the $\operatorname{Im} z$ coordinate direction at $(t, 0) \in N$ is, for each $t$ with $|t|<1+\varepsilon$, the characteristic direction, at $\tilde{H}(t, 0)$, of $\tilde{H}\left(\Sigma_{t}\right)$ in $\tilde{H}(N)$.

In fact, $\tilde{H}$ restricted to $R_{\varepsilon}$ coincides with $H$, while, if $\varepsilon>0$ is sufficiently small, the mapping $z \mapsto H(z, 0)$, defined for $z \in \mathbf{C}$ such that $|\operatorname{Re} z|<1+\varepsilon$ and $|\operatorname{Im} z|<\varepsilon$, has a complex-linear differential at every $z$ with $\operatorname{Re} z=0$ or $\operatorname{Im} z=0$. (This is clear from our definition of $H(z, 0)$ and the complex-linearity assertion in Lemma 18.3 since $H(z, 0)=f(z)$ for $z$ near 0 in $\mathbf{C}$ due to our choice of coordinates and the metric on a neighborhood of $y$.) Each of the directions in question, including those of the two curves, is characteristic, as it constitutes the intersection of the plane tangent to the respective surface with the complex line forming the image of one of the differentials just mentioned; the surfaces $Y$ and $\Sigma_{t}$, being totally real at the points of the two curves (since the tangent-plane intersections are 1-dimensional), become totally real everywhere when $\varepsilon$ is made smaller.

Let pr $: \tilde{H}(N) \rightarrow \tilde{H}\left(R_{\varepsilon}\right)$ be the mapping with $\operatorname{pr}(\tilde{H}(z, r))=\tilde{H}(z, 0)$, for $N=N_{\varepsilon}$. If $\varepsilon>0$ is sufficiently small, we extend $H: R_{\varepsilon} \rightarrow M$ to an embedding $H: N \rightarrow M$ by requiring that, for real $t, s, r$ with $|t|<1+\varepsilon$ and $s, r \in(-\varepsilon, \varepsilon)$, the image of the mapping $t \mapsto H(t, r)$ (or $s \mapsto H(t+i s, r)$ ) be a characteristic curve of 
$\tilde{H}(Y)$ (or, respectively, of $\left.\tilde{H}\left(\Sigma_{t}\right)\right)$ in $\tilde{H}(N)$, and $\operatorname{pr}(H(t+i s, r))=H(t+i s, 0)$. Such a $C^{\infty}$ extension of $H$ exists: its defining conditions mean that $t \mapsto H(t, r)$ (or $s \mapsto H(t+i s, r)$ ) is an integral curve of the unique vector field on $\tilde{H}(Y)$ (or on each $\left.\tilde{H}\left(\Sigma_{t}\right)\right)$ which is tangent to the characteristic foliation and projects under pr onto the restriction to $Y \cap R_{\varepsilon}$ of the coordinate vector field $u$ for the $\operatorname{Re} z$ direction (or, respectively, onto the restriction of $i u$ to $\Sigma_{t} \cap R_{\varepsilon}$ ).

Finally, we extend $H: N \rightarrow M$ to an embedding $H: U_{\varepsilon} \rightarrow M$, for small $\varepsilon$, by setting $H(z, w)=\exp _{H(z, \operatorname{Re} w)}[(\operatorname{Im} w) J v]$, which clearly yields (d) and (e). As the remainder of our assertion is an obvious consequence of how we chose $H$ on $N$, this completes the proof.

\section{Proof of Theorem 18.1}

We use the notation of Lemma 18.5, treating $U_{\varepsilon}$ as an open subset of both $M$ and $\mathbf{C}^{2}$. First, let us fix functions $\rho$ and $\sigma$ of the variable $t \in[-1,1]$ such that: $\sigma$ is of class $C^{\infty}$ on $[-1,1]$, has a compact support contained in $(-1,1)$, and $0 \leq \sigma \leq \sigma(0)<\varepsilon$ on $[-1,1]$, with $\sigma(0)>0$, while: $\rho$ is even, continuous on $[-1,1]$, of class $C^{\infty}$ on $(-1,1)$, with $\ddot{\rho}(0)>0$ and $\dot{\rho}>0$ on $(0,1)$, the inverse of $\rho:[0,1] \rightarrow[\rho(0), \rho(1)]$ has derivatives of all orders equal to 0 at $\rho(1)$, and $0<\rho(0) \leq \rho \leq \rho(1)<\varepsilon$ on $[-1,1]$. (We write ( ) for $d / d t$, as well as $\rho$ for $\rho(t)$, and similarly for other functions of $t$.) Let $h:[-1,1] \times S^{1} \rightarrow U_{\varepsilon}$ be the mapping given by

$$
h\left(t, e^{i \theta}\right)=(z, w) \text { with }(z, w)=(t+i \rho \cos \theta, t \rho \sin \theta+i c \sigma \sin 2 \theta) .
$$

Thus, $h$ depends on a parameter $c \in[0,1]$ and has the partial derivatives

$$
\begin{aligned}
& h_{t}=(1+i \dot{\rho} \cos \theta,(\rho+t \dot{\rho}) \sin \theta+i c \dot{\sigma} \sin 2 \theta), \\
& h_{\theta}=(-i \rho \sin \theta, t \rho \cos \theta+2 i c \sigma \cos 2 \theta) .
\end{aligned}
$$

Also, $h$ is injective except for one double point $(0,0)=h(0, \pm i)$, when $c>0$, or a curve of double points $h\left(0, e^{ \pm i \theta}\right), 0<\theta<\pi$, for $c=0$. (By (19.1), $t=\operatorname{Re} z$, and $e^{i \theta}=\alpha /|\alpha|$ for $\alpha=\operatorname{Im} z+i \operatorname{Re} w / t$, if $t \neq 0$.)

If $c>0$, the self-intersection of $h$ at $(0,0)=h(0, \pm i)$ is transverse and negative, as one easily sees using the bases, provided by (19.2), of the two real planes tangent to the image of $h$ at $(0,0)$. (Note that $\dot{\rho}(0)=\dot{\sigma}(0)=0$.)

If $c=0$ and $\left(t, e^{i \theta}\right)=(0, \pm 1)$, while $\rho, \ddot{\rho}$ stand for $\rho(0), \ddot{\rho}(0)$, then

$$
\begin{array}{ll}
\text { i) } & h_{t}=(1,0), h_{t t}=( \pm i \ddot{\rho}, 0), h_{\theta t}=(0, \pm \rho), h_{\theta \theta}=(\mp i \rho, 0), \\
\text { ii) } & h_{\theta}=h_{\theta t t}=h_{\theta \theta t}=h_{\theta \theta \theta}=(0,0)
\end{array}
$$

from (19.2) with $\dot{\rho}(0)=0$. Also, by (19.1) $-(19.2)$, for all $(t, \theta, c)$,

$$
\operatorname{Im} \mathcal{J}=2 c \sigma+\left(\rho^{2}-4 c \sigma\right) \sin ^{2} \theta+t \rho \dot{\rho}, \text { where } \mathcal{J}=z_{t} w_{\theta}-z_{\theta} w_{t} .
$$

(Thus, $\mathcal{J}$ is the Jacobian of $h$.) Hence $\operatorname{Im} \mathcal{J} \geq 0$ at any $(t, \theta)$ and for any $c \in\left[0, c_{\max }\right]$, where $c_{\max }=\min \left(1,[\rho(0)]^{2} /[4 \sigma(0)]\right)$. (In fact, the three terms $2 c \sigma,\left(\rho^{2}-4 c \sigma\right) \sin ^{2} \theta$ and $t \rho \dot{\rho}$ are all nonnegative. $)$ Moreover, the strict inequality $\operatorname{Im} \mathcal{J}>0$ clearly holds unless $c=t=0$ and $\theta \in \mathbf{Z} \pi$.

Therefore (cf. Example 9.1), $h$ with any fixed $c \in\left(0, c_{\max }\right]$ is a totally real immersion for the standard complex structure of $U_{\varepsilon} \subset \mathbf{C}^{2}$. This is also true if $c=0$, provided that one excludes the two points with $t=0$ and $\theta \in \mathbf{Z} \pi$ (at which $d h$ is not even injective; cf. (19.3) $)$. We will now show that the same conclusions 
hold for the original almost complex structure $J$ of $M$, as long as one chooses $\rho$ more carefully and $c$ is sufficiently small.

First, suppose that $c=0$. As we just saw, at $\left(t, e^{i \theta}\right)$ with $t=0$ and $\theta \notin \mathbf{Z} \pi$ the vectors $h_{t}, h_{\theta}$ are linearly independent in $\mathbf{C}^{2}$. Hence, by Lemma 18.5)(d) (case (ii)), they are also $J$-linearly independent. Now let $y=h\left(t, e^{i \theta}\right)$ with $t \neq 0$. The (disconnected) surface $K \subset M$, formed by all such $y$, is contained in the 3 -dimensional submanifold $N$ defined in Lemma 18.5. Thus, $K$ is $J$-totally real at $y$ in view of Remark 18.4 applied to a suitable totally real surface $\Sigma \subset N$, which intersects $K$ transversely in $N$ at $y$ along a noncharacteristic direction; namely, $\Sigma=Y$ if $e^{i \theta}= \pm i$ and $\Sigma=\Sigma_{t}$ otherwise. (Notation of Lemma 18.5.) That the intersection is noncharacteristic follows from (b)-(c) in Lemma 18.5. $K \cap \Sigma_{t}$, for each $t \neq 0$, is an ellipse in standard position relative to the $\operatorname{Im} z$ and $\operatorname{Re} w$ coordinate axes, while the curve $K \cap Y$ is parametrized by $t \mapsto(z, w)=(t, \pm t \rho)$, with $(t \rho)^{\circ}>0$ as $t \dot{\rho} \geq 0$ and $\rho>0$.

The next five paragraphs deal with the remaining case $c>0$.

According to Lemma 18.5(e), the $J$-complex exterior product of $u$ and $v$ trivializes the line bundle $[T M]^{\wedge 2}$ restricted to $U_{\varepsilon} \subset M$, allowing us to treat the $J$-complex exterior product of any vector fields $\xi, \xi^{\prime}$ on $U_{\varepsilon}$ as a function $U_{\varepsilon} \rightarrow \mathbf{C}$. The imaginary part $B\left(\xi, \xi^{\prime}\right)$ of that function depends on $\xi$ and $\xi^{\prime}$ only through their values at points of $U_{\varepsilon}$ (since so does the function itself); we may therefore view $B$ as a differential 2-form on $U_{\varepsilon}$, i.e., as a mapping $B: U_{\varepsilon} \rightarrow \mathcal{X}$ valued in the space $\mathcal{X}$ of skew-symmetric bilinear forms $\mathbf{C}^{2} \times \mathbf{C}^{2} \rightarrow \mathbf{R}$. Note that $B(u, v)=0$ everywhere in $U_{\varepsilon}$.

Let us now set $\Delta=B\left(h_{t}, h_{\theta}\right)$, treating $\Delta$ as a real-valued function of $(t, \theta, c)$ (where $\Delta$ depends on $c$ since $h$ does). If $\rho, \ddot{\rho}, \sigma$ denote $\rho(0), \ddot{\rho}(0)$ and $\sigma(0)$, then, for the value and partial derivatives of $\Delta$ at the points $o_{+}, o_{-} \in \mathbf{R}^{3}$ that have the $(t, \theta, c)$ coordinates $(0,0,0)$ and $(0, \pi, 0)$, we get

$$
\begin{aligned}
& \text { a) } \Delta=\Delta_{t}=\Delta_{\theta}=0, \quad \Delta_{\theta t}=\mp \rho\left(d_{u} B\right)(u, i u), \\
& \text { b) } \Delta_{c}=2 \sigma>0, \quad \Delta_{t t}=2 \rho \ddot{\rho}>0, \quad \Delta_{\theta \theta}=2 \rho^{2}>0, \\
& \text { c) } B_{\theta}=B_{c}=0 \text { and } B_{t}=d_{u} B \text { at } o_{ \pm} .
\end{aligned}
$$

Here $B_{t}, B_{\theta}, B_{c}$ are the $\mathcal{X}$-valued partial derivatives of $B \circ h$, while $u$ denotes the $\operatorname{Re} z$ coordinate vector field, $d_{u} B: U_{\varepsilon} \rightarrow \mathcal{X}$ is the corresponding directional derivative of $B: U_{\varepsilon} \rightarrow \mathcal{X}$, and $i$ in $i u$ refers to the complex structure of $\mathbf{C}^{2}$.

In fact, we have (19.5) c) and $\Delta=0$ at $o_{ \pm}$as $h_{\theta}=h_{c}=0$ and $h_{t}=u$ at $o_{ \pm}$by (19.3) and (19.1). Now let $A$ be the analogue of $B$ obtained by using, instead of $J$, the complex structure of $\mathbf{C}^{2}$. Hence $A$ is constant as a function $U_{\varepsilon} \rightarrow \mathcal{X}$, while, by Lemma 18.5(d), $B=A$ at all points of $U_{\varepsilon}$ that have the form $(t, 0)$ or $(i s, 0)$ with $t, s \in \mathbf{R}$, including, when $c=0$, the $h$-images $(0, \pm \rho(0))$ of $\left(t, e^{i \theta}\right)=(0, \pm 1)$. (Also, $A\left(h_{t}, h_{\theta}\right)=\operatorname{Im} \mathcal{J} ;$ cf. (19.4).) To evaluate the partial derivatives of $\Delta$ at $o_{ \pm}$ in (19.5), we differentiate the three-factor "product" $B\left(h_{t}, h_{\theta}\right)$ via the Leibniz rule. The partial derivatives in question differ from those of $A\left(h_{t}, h_{\theta}\right)$ just by the extra terms in which the $B$ factor is differentiated. However, by (19.3) ii), such an extra term can only be nonzero if exactly one differentiation falls on the $h_{\theta}$ factor. This, combined with (19.5) c), shows that the extra terms are all zero, except, possibly, those in $\Delta_{t t}$ and $\Delta_{\theta t}$, equal to $\pm 2 \rho\left(d_{u} B\right)(u, v)$ and, respectively, $\mp \rho\left(d_{u} B\right)(u, i u)$. (By (19.3) i), $h_{t}=u, h_{\theta t}= \pm \rho v$ and $h_{\theta \theta}=\mp \rho i u$ at $o_{ \pm}$.) Now (19.5) follows, since $B(u, v)=0$ identically on $U_{\varepsilon}$, and so $\left(d_{u} B\right)(u, v)=d_{u}[B(u, v)]=0$, while 
the partial derivatives of $A\left(h_{t}, h_{\theta}\right)$ at $o_{ \pm}$are easily found using the Leibniz rule and (19.3): for instance, $\left[A\left(h_{t}, h_{\theta}\right)\right]_{t}=A\left(h_{t}, h_{\theta t}\right)= \pm \rho(0) A(u, v)=0$.

Our functions $\rho$ and $\sigma$ have been so far subject to the specific conditions listed above, but otherwise arbitrary. However, a special choice of $\rho$ gives

$$
\Delta_{t t} \Delta_{\theta \theta}-\left(\Delta_{\theta t}\right)^{2}>0 \text { at the two points }(t, \theta, c)=o_{ \pm} .
$$

Namely, by (19.5),$\Delta_{t t} \Delta_{\theta \theta}-\left(\Delta_{\theta t}\right)^{2}=4 \rho^{3} \ddot{\rho}-\left[\rho\left(d_{u} B\right)(u, i u)\right]^{2}$. Although the function $\left(d_{u} B\right)(u, i u): U_{\varepsilon} \rightarrow \mathbf{R}$ does not depend on the choice of $\rho$, its value used here does, as it is taken at the point $h(0, \pm 1)=( \pm i \rho(0), 0)$. Modifying $\rho$ so as to keep $\rho(0)>0$ unchanged while making $\ddot{\rho}(0)$ so large that $4 \rho(0) \ddot{\rho}(0)>\left[\left(d_{u} B\right)(u, i u)\right]^{2}$ at $( \pm i \rho(0), 0)$, we obtain (19.6).

As $\Delta_{c}>0=\Delta$ at $o_{ \pm}$(see (19.5)), the implicit function theorem applied to the function $(t, \theta, c) \mapsto \Delta$ shows that, in some neighborhood of either point $o_{ \pm}$in $\mathbf{R}^{3}$, the equation $\Delta=0$ describes the graph of a $C^{\infty}$ function $(t, \theta) \mapsto c$, equal to 0 at $(0,0)$ or, respectively, at $(0, \pi)$. Partial differentiations of the equality $\Delta=0$ with respect to $t, \theta$ show that, as a consequence of (19.5) and (19.6), 0 is a nondegenerate local-maximum value of the function $(t, \theta) \mapsto c$ at $(0,0)$ (or $(0, \pi))$. Thus, we may choose neighborhoods of $(0,0)$ and $(0, \pi)$ on which $\Delta=B\left(h_{t}, h_{\theta}\right)$ is nonzero everywhere for all sufficiently small $c>0$. Due to the definition of $B$ and Example 9.1, this shows that $h$, with any such fixed $c$, is a $J$-totally real immersion when restricted to the two corresponding neighborhoods of $(0, \pm 1)$ in $[-1,1] \times S^{1}$. On the other hand, removing the latter two neighborhoods from $[-1,1] \times S^{1}$, we are left with a compact set on which $h$ is $J$-totally real for any small $c>0$ (since that is the case for $c=0$, as we saw earlier).

Thus, our $h$, with any fixed $c>0$ close to 0 , is a $J$-totally real immersion with a single, transverse, negative self-intersection, while $U_{\varepsilon}$ is an obvious choice of the open subset required in (v). The only claim that still needs proving is smoothness of the union $S$ of the image of $h$ with the complements of the disks $D^{ \pm}$of radius $\rho(1)$, centered at $( \pm 1,0)$, in the Euclidean planes $\left\{(z, w) \in \mathbf{C}^{2}: \operatorname{Re} z \mp 1=\operatorname{Im} w=0\right\}$. To this end, let us fix $\delta \in(0,1)$ with $\operatorname{supp} \sigma \subset[-\delta, \delta]$, and denote by $N^{\prime}$ the disconnected 3-manifold formed by all $(z, w) \in \mathbf{C}^{2}$ with $|\operatorname{Re} z|>\delta$ and $\operatorname{Im} w=0$. Smoothness of $S$ now follows from smoothness of the (disconnected) surface $\Sigma^{\prime}$ in $N^{\prime}$ which consists of $(z, w) \in N^{\prime}$ such that, setting $t=\operatorname{Re} z$ and defining $r \geq 0$ by $r^{2}=(\operatorname{Im} z)^{2}+(\operatorname{Re} w)^{2}$, one has either $|t|=1$ and $r \geq \rho(1)$ or $\delta<|t|<1$ and $r=\rho(t)$. In fact, $\Sigma^{\prime}$ is a smooth surface in the Euclidean 3 -space $\mathbf{C} \times \mathbf{R} \subset \mathbf{C}^{2}$ with the coordinates $\operatorname{Re} z, \operatorname{Im} z, \operatorname{Re} w$, since it is obtained by revolving, about the $\operatorname{Re} z$ axis, a (disconnected) $C^{\infty}$ curve lying in the half-plane $\operatorname{Im} z>0=\operatorname{Re} w$. The curve is the intersection of $\Sigma^{\prime}$ with the half-plane, so that its smoothness becomes obvious if one parametrizes either of its components using $r$ (the distance from the $\operatorname{Re} z$ axis) as the parameter, and recalls the assumptions made earlier about the derivatives of the inverse of $\rho$. Finally, the set $S^{\prime}=S \cap N^{\prime}$ is relatively open in $S$ (as $S^{\prime}=\{(z, w) \in S:|\operatorname{Re} z|>\delta\}$ ), while $S^{\prime}$ is also the image of $\Sigma^{\prime}$ under the diffeomorphism $N^{\prime} \rightarrow N^{\prime}$ sending $(z, w)$ to $(z,(\operatorname{Re} z) w)$.

The proof of Theorem 18.1 is now complete.

\section{Totally Real tori and Klein Bottles in $\mathbf{C}^{2}$}

The torus $T^{2}$ and Klein bottle $K^{2}$ both admit totally real embeddings in $\mathbf{C}^{2}$. For $T^{2}$ this is obvious ((v) in Section 9). Rudin [25] first found such embeddings 
of $K^{2}$. In this section we describe further examples of totally real immersions and embeddings of $T^{2}$ and $K^{2}$ in $\mathbf{C}^{2}$.

For $C^{1}$ functions $x, y: U \rightarrow \mathbf{C}$ on an open set $U$ in the $(t, s)$-plane $\mathbf{R}^{2}$, we define the function $\mathcal{J}(x, y): U \rightarrow \mathbf{C}$ by $\mathcal{J}(x, y)=x_{s} y_{t}-x_{t} y_{s}$ (cf. Example 9.1); the subscripts stand for the partial derivatives.

Example 20.1. Let $x, y, h: \mathbf{R}^{2} \rightarrow \mathbf{C}$ be doubly $2 \pi$-periodic $C^{1}$ functions of the variables $t, s$ such that $|\mathcal{J}(x, y)|$ is bounded on $\mathbf{R}^{2}$ and $h_{s}=0$ identically, while $\left|x_{s} h_{t}\right| \geq \varepsilon$ for some real number $\varepsilon>0$. The mapping $(x, y+a h): \mathbf{R}^{2} \rightarrow \mathbf{C}^{2}$, with any constant $a \in \mathbf{R}$, then descends to the torus $T^{2}=[\mathbf{R} / 2 \pi \mathbf{Z}] \times[\mathbf{R} / 2 \pi \mathbf{Z}]$, and, for large $|a|$, it produces a totally real immersion $f: T^{2} \rightarrow \mathbf{C}^{2}$. If, in addition, $x, y, h$ are all invariant under the transformation $(t, s) \mapsto(t+\pi,-s)$ of $\mathbf{R}^{2}$, then $(x, y+a h)$ further descends to a totally real immersion $f: K^{2} \rightarrow \mathbf{C}^{2}$ of the Klein bottle $K^{2}=\mathbf{R}^{2} / \Gamma=T^{2} / \mathbf{Z}_{2}$, where $\Gamma$ is the transformation group generated by $\Phi$ and $\Psi$ with $\Phi(t, s)=(t+\pi,-s)$ and $\Psi(t, s)=(t, s+2 \pi)$.

In fact, $(x, u): \mathbf{R}^{2} \rightarrow \mathbf{C}^{2}$ is a totally real immersion if and only if $\mathcal{J}(x, u) \neq 0$ everywhere in $\mathbf{R}^{2}$ (see Example 9.1). Clearly, for any $r \in \mathbf{R}$,

$$
\mathcal{J}(x, y+a h)=\mathcal{J}(x, y)+a x_{s} h_{t},
$$

and so $|\mathcal{J}(x, y+a h)| \rightarrow \infty$ as $a \rightarrow \infty$, uniformly on $U$. Therefore, if $|a|$ is sufficiently large, $\mathcal{J}(x, y+a h) \neq 0$ everywhere in $\mathbf{R}^{2}$.

Example 20.2. The assumptions listed in Example 20.1 are obviously satisfied by the functions $x(t, s)=e^{i k t}(\sin s+i \sin 2 s), y(t, s)=e^{i l t} \cos s, h(t, s)=e^{i l t}$, where $k$ and $l$ are fixed integers with $l \neq 0$. The mapping $(x, y+a h): \mathbf{R}^{2} \rightarrow \mathbf{C}^{2}$ thus descends, for large $a$, to a totally real immersion $f=f^{k, l}: T^{2} \rightarrow \mathbf{C}^{2}$ of the 2 -torus. In the case where $k$ is odd and $l$ is even, it similarly descends to a totally real immersion $f=f^{k, l}: K^{2} \rightarrow \mathbf{C}^{2}$ of the Klein bottle.

Furthermore, if $k, l$ are integers and either

(a) $l=1$, while $\Sigma$ is the 2 -torus $T^{2}$, or

(b) $l=2$, while $k$ is odd and $\Sigma$ is the Klein bottle $K^{2}$,

then, for all sufficiently large $a>1$, the mapping $f^{k, l}: \Sigma \rightarrow \mathbf{C}^{2}$ defined above is a totally real embedding.

In fact, injectivity of $f^{k, l}$ follows since $(x, u)=(x(t, s), y(t, s)+a h(t, s))$ determines $(\alpha, \beta)=\left(e^{i s}, e^{i t}\right)$ either uniquely (case (a)) or up to the involution $(\alpha, \beta) \mapsto(\bar{\alpha},-\beta)$ (case (b)). Specifically, $\cos s=|u|-a, e^{i l t}=u /|u|$ and $\sin s=x e^{-i k t}(1+2 i \cos s)^{-1}$.

\section{Proofs of Theorem 2.3 and Corollaries $2.4-2.9$}

Let $M$ be an almost complex surface. Corollary 11.3 implies that $S^{2}$ admits a totally real immersion in $M$, while totally real embeddings $T^{2} \rightarrow M$ and $K^{2} \rightarrow M$ exist in view of Proposition 10.1 combined with Example 20.2.

If real surfaces $\Sigma$ and $\Sigma^{\prime}$ admit totally real immersions $f$ and $f^{\prime}$ in $M$, then totally-real immersibility of $\Sigma \# \Sigma^{\prime}$ in $M$ is an obvious consequence of Theorem 18.1 applied to $M \backslash\left[f(\partial D) \cup f^{\prime}\left(\partial D^{\prime}\right)\right]$ rather than $M$, and $x^{ \pm} \in M$ chosen so that $f(x)=x^{+} \neq x^{-}=f^{\prime}\left(x^{\prime}\right)$ for some $x \in \Sigma, x^{\prime} \in \Sigma^{\prime}$, along with $\Sigma^{+}=f(\operatorname{Int} D)$ and $\Sigma^{-}=f^{\prime}\left(\operatorname{Int} D^{\prime}\right)$, where $D, D^{\prime}$ are small closed disks embedded in $\Sigma, \Sigma^{\prime}$ so as to contain $x, x^{\prime}$ as interior points. 
Next, let $\Sigma^{+}$be a closed totally real surface embedded in $M$. A totally real 2 torus $\Sigma^{-}$embedded in $M \backslash \Sigma^{+}$exists by Corollary 10.2 in view of Theorem 18.1. $\Sigma^{+} \# \Sigma^{-} \approx \Sigma \# T^{2}$ admits a totally real immersion in $M$ with just one self-intersection in the form of a double point at which the self-intersection is transverse and, if $\Sigma$ is orientable, also negative.

The statement following Lemma 16.2, applied to this immersed image of $\Sigma \# T^{2}$ (rather than $\Sigma$ ), gives rise to a totally real embedding of $\Sigma \# T^{2} \# K^{2}$ in $M$, thus proving Theorem 2.3 ,

Corollaries 2.4 - 2.6 now follow, and Corollary 2.7 is easily derived from Corollary 2.6 using a real form $\mathbf{R P}^{2} \subset \mathbf{C P}^{2}$ (see (vi) in Section 9).

Corollary 2.8 for $T^{2}$ and $S^{2}$ is clear from Theorem 2.3, Lemma 12.1(a) and Example 13.4 (or, Example 13.5) combined with Remark 13.1. To prove Corollary 2.8 for nonorientable closed surfaces $\Sigma$, note that the operation $\Sigma \mapsto \Sigma \# T^{2} \# K^{2}$ (where $K^{2}$ is the Klein bottle) reduces the Euler characteristic by 4 , and so, in view of Theorem 2.3, one need only to show the existence of a totally real embedding in $M=\mathbf{C P}^{2} \# m \overline{\mathbf{C P}^{2}}, m \geq 1$, of any closed nonorientable surface $\Sigma$ such that $\chi(\Sigma) \in\{-2,-1,0,1\}$. If $\chi(\Sigma) \in\{-2,0\}$, this follows from Theorem 2.3 and embeddability of $S^{2}$, as $\Sigma=S^{2} \# T^{2} \# K^{2}$. Now let $\chi(\Sigma)= \pm 1$. Thus, $\Sigma=\mathbf{R P}^{2}$ or $\Sigma=3 \mathbf{R P}^{2}$, and our claim is obvious from (vi) in Section 9 Lemma 12.1(a) and Corollary 12.2 .

Finally, Corollary 2.9 is immediate from Corollary 2.4, (5.3) a) and (1.7).

\section{ObStRuCtions FOR EMBEDDED ORIENTABLE SURFACES}

Let $M$ be a compact almost complex surface. If a closed oriented real surface $\Sigma$ admits a totally real embedding $f: \Sigma \rightarrow M$ and $\mu \in H^{2}(M, \mathbf{R})$ denotes the (real) Poincaré dual of $f_{*}[\Sigma] \in H_{2}(M, \mathbf{R})$, setting $\chi=\chi(\Sigma)$ and $c_{1}=c_{1}(M)$ we can rewrite (5.4), with $n=2$, and (5.3) b) as

$$
\mu \smile \mu=-\chi, \quad \mu \smile c_{1}=0 .
$$

For any closed oriented 4-manifold $M$, the cup product $\smile$ is nondegenerate as a real-valued symmetric bilinear form in $H^{2}(M, \mathbf{R})$, and its sign pattern consists of $b^{+}$pluses and $b^{-}$minuses, for some $b^{ \pm} \in \mathbf{Z}$ with $b_{2}(M)=b^{+}+b^{-}$.

We have the following easy result.

Proposition 22.1. Let $M$ be a closed almost complex surface for which $b^{+}=1$, $c_{1} \smile c_{1} \geq 0$ and $c_{1} \neq 0$ in $H^{2}(M, \mathbf{R})$, and let a closed orientable surface $\Sigma$ admit a totally real embedding $f: \Sigma \rightarrow M$.

(a) $\Sigma$ must then be diffeomorphic to the torus $T^{2}$ or the sphere $S^{2}$.

(b) If, in addition, $M$ has $b^{-}=0$, then $\Sigma$ is diffeomorphic to $T^{2}$.

(c) If $\Sigma$ is diffeomorphic to $T^{2}$, then either $c_{1} \smile c_{1}>0$ and $f_{*}[\Sigma]=0$ in

$H_{2}(M, \mathbf{R})$, or $c_{1} \smile c_{1}=0$ and $f_{*}[\Sigma]$ is a real multiple of the Poincaré dual of $c_{1}$ in $H_{2}(M, \mathbf{R})$.

Proof. If $\chi \leq 0$, relations (22.1) imply that the cup-product form $\smile$ is positive semidefinite on the subspace $W \subset H_{2}(M, \mathbf{R})$ spanned by $\mu$ and $c_{1}$. Since $\smile$ has the Lorentzian sign pattern $+-\ldots-$, this shows that $\operatorname{dim} W=1$. Using (22.1), we now obtain (c). Also, as the inequality $\chi<0$ would, by (22.1), make $\mu$ and $c_{1}$ linearly independent (and hence yield $\operatorname{dim} W=2$ ), we see that $\chi=\chi(\Sigma) \geq 0$, 
which proves (a). Finally, condition $b^{-}=0$ implies that $c_{1}$ spans $H^{2}(M, \mathbf{R})$ so that (22.1) gives $\mu=0$ and $\chi=0$, which yields (b). This completes the proof.

Our next conclusion is an immediate consequence of Proposition 22.1,

Corollary 22.2. The torus $T^{2}$ is the only closed orientable real surface that admits a totally real embedding in $\mathbf{C}^{2}, \mathbf{C P}^{2}$ or the complex surface obtained by blowing up a point in $\mathbf{C P}^{2}$.

Proof. A totally real embedding of $T^{2}$ exists according to (v) in Section 9 . Concerning its nonexistence for other real surfaces, the case of $\mathbf{C}^{2}$ follows from that of $\mathbf{C P}^{2}$ via the inclusion $\mathbf{C}^{2} \subset \mathbf{C P}^{2}$ (or, directly from (5.4) with $H_{2}\left(\mathbf{C}^{2}, \mathbf{Z}\right)=\{0\}$ ). As for $M=\mathbf{C P}^{2}$ or $M=\mathbf{C P}^{2} \# \overline{\mathbf{C P}^{2}}$, the only other possibility left by Proposition 22.1(a) is that of a totally real 2-sphere embedded in $M$. This in turn is excluded by Corollary 5.2. (For $\mathbf{C P}^{2}$, we may also use Proposition 22.1(b).)

Proofs of Corollaries 2.10 and 2.11. In both corollaries, the nonexistence part is obvious from Proposition 22.1 and Corollary 2.9. (Note that condition $m \leq 9$ in Corollary 2.11 amounts to $c_{1} \smile c_{1} \geq 0$; cf. Section 30.) Corollary 2.11 is now immediate from Corollary 2.8. As for Corollary 2.10, the existence assertion for $T^{2}$ is clear from (v) in Section 9, and for $S^{2}$ it is provided by (vii) in Section 9. Finally, let $\Sigma$ be a nonorientable closed surface. Since the operation $\Sigma \mapsto \Sigma \# T^{2} \# K^{2}$ (where $K^{2}$ is the Klein bottle) reduces the Euler characteristic of any closed surface by 4 , by Theorem 2.3 we just need to show the existence of a totally real embedding $\Sigma \rightarrow M=\mathbf{C P}^{1} \times \mathbf{C P}^{1}$ under the additional assumption that $\chi(\Sigma) \in\{-2,0\}$. Now, if $\chi(\Sigma)=0$, this follows from Corollary 2.5. For $\chi(\Sigma)=-2$, we have $\Sigma=$ $S^{2} \# T^{2} \# K^{2}$, and so we may use the embeddability of $S^{2}$ along with Theorem 2.3 .

\section{EMBEDdings OF NONORIENTABLE SURFACES}

The self-intersection formula (5.5) often fails to detect nonembeddability of nonorientable surfaces. For instance, the genus 3 surface $\mathbf{R} P^{2} \# \mathbf{R} P^{2} \# \mathbf{R} P^{2}$ admits no totally real embedding in $\mathbf{C P}^{2}$, yet this fact cannot be derived from (5.5). To obtain useful obstructions for totally real embeddings of nonorientable closed manifolds one needs more subtle intersection-theoretic tools, such as the Pontryagin square operation, applicable to this case via a result of Massey described below. Our presentation follows 23 and 4 .

Given a manifold $M$ and an integer $k \geq 0$, the Pontryagin square [23] is a natural cohomology operation $H^{k}\left(M, \mathbf{Z}_{2}\right) \rightarrow H^{2 k}\left(M, \mathbf{Z}_{4}\right)$ which, applied to $\bmod 2$ reductions of integral classes, assigns the value $[\xi \smile \xi \bmod 4]$ to $[\xi \bmod 2]$, for any $\xi \in H^{k}(M, \mathbf{Z})$. We use the symbol $\mu^{2}$ for the Pontryagin square of $\mu \in H^{n}\left(M, \mathbf{Z}_{2}\right)$ in the case of a closed oriented manifold $M$ of dimension $2 n$ (such as a closed almost complex manifold with $\operatorname{dim}_{\mathbf{C}} M=n$ ). Thus, $\mu^{2} \in \mathbf{Z}_{4}$. Also, by definition, $\mu^{2}=0 \in \mathbf{Z}_{4}$ if $M$ is diffeomorphic to $\mathbf{C}^{n}$. We begin with the following special case of a result of Massey [23, Theorem 1]:

Lemma 23.1. Given an embedding $f: \Sigma \rightarrow M$ of a closed manifold $\Sigma$ in a closed oriented manifold $M$ with $\operatorname{dim} M=2 \operatorname{dim} \Sigma=2 n$, where $n$ is even and $n \geq 2$, let $\mu \in H^{n}\left(M, \mathbf{Z}_{2}\right)$ be the Poincaré dual of $f_{*}[\Sigma] \in H_{n}\left(M, \mathbf{Z}_{2}\right)$. If $\chi(\nu) \in \mathbf{Z}$ and $\mu^{2} \in \mathbf{Z}_{4}$ are the twisted Euler number of the normal bundle $\nu$ of $f$ and the Pontryagin square of $\mu$, then

$$
\mu^{2}=[\chi(\nu) \bmod 4]+2\left[w_{1} \smile w_{n-1}\right] \in \mathbf{Z}_{4}, \quad \text { where } w_{j}=w_{j}(\Sigma),
$$


$2\left[w_{1} \smile w_{n-1}\right]$ being the image of $w_{1} \smile w_{n-1}$ under the nontrivial coefficient homomorphism $\mathbf{Z}_{2} \rightarrow \mathbf{Z}_{4}$.

Proof. Any embedding $f: \Sigma \rightarrow M$ gives rise to the associated mapping $F$ from $M$ into the Thom space $\operatorname{Th}(\nu)$ (that is, a one-point compactification) of the normal bundle $\nu$ of $f$. The Thom space is an oriented pseudomanifold, and with the usual orientation conventions, $F$ induces the identity homomorphism between the top (co)homology groups. Moreover, $\mu$ is the $F$-pullback of $[\mathcal{U} \bmod 2] \in H^{n}\left(\operatorname{Th}(\nu), \mathbf{Z}_{2}\right)$, that is, of the (well-defined) mod 2 reduction of the Thom class $\mathcal{U}$. (The Thom class itself lives in the cohomology of another pair with twisted $\mathbf{Z}$ coefficients.) As shown by Massey [23, Theorem 1], the Pontryagin square $[\mathcal{U} \bmod 2]^{2}$ is the image, under the Thom isomorphism, of $[\mathrm{e}(\nu) \bmod 4]+2\left[w_{1} \smile w_{n-1}\right]$, where $[\mathrm{e}(\nu) \bmod 4]$ denotes the mod 4 reduction of the twisted Euler class of $\nu$. Now (23.1) follows since naturality of the Pontryagin square allows us to compute the right-hand side of (23.1) in the cohomology of either $\operatorname{Th}(\nu)$ or $M$, with both sides treated as elements of $\mathbf{Z}_{4}$.

Corollary 23.2. Let $f: \Sigma \rightarrow M$ be a totally real embedding of a closed real manifold $\Sigma$ in a closed almost complex manifold $M$ with $\operatorname{dim}_{\mathbf{R}} \Sigma=\operatorname{dim}_{\mathbf{C}} M=n$, where $n$ is even, and let $\mu \in H^{n}\left(M, \mathbf{Z}_{2}\right)$ be the Poincaré dual of the homology class $f_{*}[\Sigma] \in H_{n}\left(M, \mathbf{Z}_{2}\right)$. The Pontryagin square $\mu^{2} \in \mathbf{Z}_{4}$ is then characterized by (23.1). For $n=2$, this becomes

$$
\mu^{2}=[\chi(\Sigma) \bmod 4] .
$$

Equality (23.2) remains valid, with $\mu^{2}=0$, if $M$, rather than being compact, is assumed diffeomorphic to $\mathbf{C}^{2}$.

This is obvious from (5.1) and Wu's formula (1.7); if $M \approx \mathbf{C}^{2}$, we may use Lemma 23.1 with $M$ replaced by its one-point compactification $S^{4}$.

A further obvious consequence can be stated as follows.

Corollary 23.3. Suppose that a closed real surface $\Sigma$ admits a totally real embedding in the complex surface $M=\mathbf{C P}^{2}$ or $M=\mathbf{C P}^{2} \# \overline{\mathbf{C P}^{2}}$. Defining $\chi_{4} \in\{0,1,2,3\}$ by $\chi_{4} \equiv \chi(\Sigma) \bmod 4$, we then have $\chi_{4} \in\{0,1\}$ if $M=\mathbf{C P}^{2}$ and $\chi_{4} \in\{0,1,3\}$ if $M=\mathbf{C P}^{2} \# \overline{\mathbf{C P}^{2}}$.

In fact, as in the argument for Proposition [5.1, this is immediate from (23.2), since, for integers $p, q$, the $\bmod 4$ congruence class of $p^{2}$ must contain 0 or 1 , while that of $p^{2}-q^{2}$ must contain 0,1 or -1 .

Proofs of Corollaries 2.12 - 2.14. The nonexistence assertion for orientable surfaces $\Sigma$ other than $T^{2}$ is immediate from Corollary 22.2, while the case of $T^{2}$ is settled by Corollary 2.5. Now, let the surface $\Sigma$ be nonorientable. The operation $\Sigma \mapsto \Sigma \# T^{2} \# K^{2}$ (with $K^{2}$ denoting the Klein bottle) reduces $\chi(\Sigma)$ by 4 . Thus, by Theorem 2.3, for the existence assertions in Corollaries 2.12-2.14 it suffices to show that a totally real embedding exists if $\chi(\Sigma)$ is zero, or $\chi(\Sigma) \in\{0,1\}$ or, respectively, $\chi(\Sigma) \in\{-1,0,1\}$. For $\chi(\Sigma)=0$ in all three corollaries, or $\chi(\Sigma)=1$ in Corollary 2.13, or $\chi(\Sigma)= \pm 1$ in Corollary 2.14, this existence statement is clear from Corollary 2.5, or (vi) in Section 9 or, respectively, (vi) in Section 9 combined with Corollary 12.2, as $\chi(\Sigma)=-1$ for $\Sigma=\mathbf{R P}^{2} \# \mathbf{R} \mathbf{P}^{2} \# \mathbf{R} \mathbf{P}^{2}$. 
Finally, the nonexistence statements for nonorientable $\Sigma$ can be established as follows. Let $\Sigma$ admit a totally real embedding in $M$. Assume first that $M=\mathbf{C P}^{2}$ or $M=\mathbf{C P}^{2} \# \overline{\mathbf{C P}^{2}}$. By Corollary 23.3. $\chi(\Sigma)$ has the required remainder mod 4 . Next, if $M=\mathbf{C}^{2}$, this last fact yields $\chi(\Sigma) \equiv 0 \bmod 4$, since $\mathbf{C}^{2} \subset \mathbf{C P}^{2}$ and $\chi(\Sigma)$ is even by Corollary 2.9 .

\section{The SETS $\mathfrak{I}_{q}(\Sigma)$ AND $\mathfrak{D}_{ \pm}^{\varepsilon}(M)$ IN SPECIAL CASES}

Given a real manifold $\Sigma$ and $q \in\{2,4, \ldots, \infty\}$, let $\mathfrak{I}_{q}(\Sigma)$ be the set defined by (2.9), with $\mathbf{Z}_{\infty}=\mathbf{Z}$. Thus, as $H^{1}\left(S^{n}, \mathbf{Z}_{q}\right)=\{0\}$ for $n \geq 2$,

$$
\mathfrak{I}_{q}\left(S^{n}\right)=\{0\} \quad \text { whenever } \quad n \geq 2 \quad \text { and } \quad q \in\{2,4,6, \ldots, \infty\} .
$$

Since $\mathfrak{I}_{q}(\Sigma)$ is either empty or forms a coset of the subgroup of $H^{1}\left(\Sigma, \mathbf{Z}_{q}\right)=$ $\operatorname{Hom}\left(\pi_{1} \Sigma, \mathbf{Z}_{q}\right)$ consisting of all homomorphisms valued in the even subgroup $2 \mathbf{Z}_{q}$ (the image of $\mathbf{Z}_{q}$ under the homomorphism $\xi \mapsto 2 \xi$ ), we also have

$$
\mathfrak{I}_{q}\left(T^{n}\right)=\left(2 \mathbf{Z}_{q}\right)^{n} \subset\left(\mathbf{Z}_{q}\right)^{n}=H^{1}\left(T^{n}, \mathbf{Z}_{q}\right),
$$

()$^{n}$ in $\left(2 \mathbf{Z}_{q}\right)^{n},\left(\mathbf{Z}_{q}\right)^{n}$ being the $n$th Cartesian power, with $H^{1}\left(T^{n}, \mathbf{Z}_{q}\right)=\left(\mathbf{Z}_{q}\right)^{n}$ due to the standard identification resulting from (1.5) (where $\pi_{1} T^{n}=\mathbf{Z}^{n}$ ). Next, one easily verifies that, for all $q \in\{2,4,6, \ldots, \infty\}$,

$$
\mathfrak{I}_{q}\left(\mathbf{R P}^{n}\right)= \begin{cases}\{\varphi\} & \text { if } q / 2 \text { is finite and odd } \\ \varnothing & \text { if } q=\infty \text { or } q / 2 \text { is even }\end{cases}
$$

where $\varphi \in H^{1}\left(\mathbf{R P}^{n}, \mathbf{Z}_{q}\right)=\operatorname{Hom}\left(\mathbf{Z}_{2}, \mathbf{Z}_{q}\right)$ (cf. (1.5)) is the unique nonzero homomorphism $\mathbf{Z}_{2} \rightarrow \mathbf{Z}_{q}$. (Note that $\pi_{1}\left[\mathbf{R P} P^{n}\right]=\mathbf{Z}_{2}$, and $w_{1}\left(\mathbf{R} P^{n}\right.$ ) with (1.6) equals Id $: \mathbf{Z}_{2} \rightarrow \mathbf{Z}_{2}$.)

For an almost complex manifold $M$, a fixed sign \pm , and $\varepsilon \in\{0,1\}$, let $\mathfrak{D}_{ \pm}^{\varepsilon}(M)$ now be defined by (2.8). If $M=\mathrm{CP}^{2}$, we thus have

$$
\mathfrak{D}_{-}^{1}\left(\mathbf{C P}^{2}\right)=\varnothing, \mathfrak{D}_{+}^{1}\left(\mathbf{C P}^{2}\right)=\mathfrak{D}_{+}^{0}\left(\mathbf{C P}^{2}\right)=\{0\}, \mathfrak{D}_{-}^{0}\left(\mathbf{C P}^{2}\right)=\left\{\left[\mathbf{R P}^{2}\right]\right\},
$$

$\left[\mathbf{R P}^{2}\right]$ being the $\mathbf{Z}_{2}$-homology class of $\Sigma=\mathbf{R} \mathbf{P}^{n}$ in (vi) of Section 9 for $n=$ 2. (In fact, $c_{1}(M): H_{2}(M, \mathbf{Z}) \rightarrow \mathbf{Z}$ and $w_{2}(M): H_{2}\left(M, \mathbf{Z}_{2}\right) \rightarrow \mathbf{Z}_{2}$ then are isomorphisms.) Finally, since $H_{2}\left(\mathbf{C}^{2}, \mathbf{Z}_{[2]}\right)=0$,

$$
\mathfrak{D}_{+}^{1}\left(\mathbf{C}^{2}\right)=\{0\}, \quad \mathfrak{D}_{+}^{0}\left(\mathbf{C}^{2}\right)=\{0\}, \quad \mathfrak{D}_{-}^{0}\left(\mathbf{C}^{2}\right)=\mathfrak{D}_{-}^{1}\left(\mathbf{C}^{2}\right)=\varnothing .
$$

\section{Closed Surfaces AND COHOMOlOGy}

We begin with some known facts gathered here for easy reference. First, the fundamental group $\Gamma=\pi_{1} K^{2}$ of the Klein bottle $K^{2}=\mathbf{R P}^{2} \# \mathbf{R} \mathbf{P}^{2}$, treated as a group of deck transformations in $\mathbf{R}^{2}$, has the generators $\Phi, \Psi$ described in Example 20.1. Since $\Psi^{2}=\Psi \Phi \Psi^{-1} \Phi^{-1}$, the Abelianization $H_{1}\left(K^{2}, \mathbf{Z}\right)$ of $\Gamma$ may from now on be identified with the direct product $\mathbf{Z} \times \mathbf{Z}_{2}$ (the factor groups being generated by $\Phi$ and $\Psi$ ). As $\Psi$ is orientation-preserving while $\Phi$ is not, $w_{1}\left(K^{2}\right): \pi_{1} K^{2} \rightarrow \mathbf{Z}_{2}=\{0,1\}$ sends $\Phi$ to 1 and $\Psi$ to 0 (cf. (1.6)). The homomorphism $\mathbf{Z} \times \mathbf{Z}_{2} \rightarrow \mathbf{Z}_{2}$, arising as $w_{1}\left(K^{2}\right)$ descends to $H_{1}\left(K^{2}, \mathbf{Z}\right)$, therefore sends $(k, \varepsilon)$ to $[k \bmod 2]$. Finally, the transformation $(t, s) \mapsto(-t, s)$ in $\mathbf{R}^{2}$ commutes with $\Psi$ and conjugates $\Phi$ with $\Phi^{-1}$, so that it descends to a diffemorphism $K^{2} \rightarrow K^{2}$ of $K^{2}=\mathbf{R}^{2} / \Gamma$, which acts in $H_{1}\left(K^{2}, \mathbf{Z}\right)=\mathbf{Z} \times \mathbf{Z}_{2}$ by $(k, \varepsilon) \mapsto(-k, \varepsilon)$. 
Remark 25.1. For a closed real surface $\Sigma$ of genus $s$ we have, up to a diffeomorphism, one of three cases:

(a) $\Sigma=T^{2} \# \ldots \# T^{2}$ ( $s$ summands),

(b) $s$ is even and $\Sigma=T^{2} \# \ldots \# T^{2} \# K^{2}$, with $s / 2$ summands,

(c) $s$ is odd and $\Sigma=T^{2} \# \ldots \# T^{2} \# \mathbf{R} P^{2}$, with $(s+1) / 2$ summands.

This includes $\Sigma=S^{2}$ (case (a), with $s=0$ ), the Klein bottle $\Sigma=K^{2}$ (case (b) with $s=2$ ), and $\Sigma=\mathbf{R P}^{2}$ (case (c), for $s=1$ ).

Remark 25.2. Let $G, G_{1}, \ldots, G_{k}$ be Abelian groups and let $\Sigma$ be a manifold with $H_{1}(\Sigma, \mathbf{Z})=G_{1} \times \ldots \times G_{k}$. Then, by (1.5) and Remark 1.1.

(i) $H^{1}(\Sigma, G)=\operatorname{Hom}\left(G_{1}, G\right) \times \ldots \times \operatorname{Hom}\left(G_{k}, G\right)$,

(ii) the coefficient-reduction homomorphism $H^{1}(\Sigma, G) \rightarrow H^{1}\left(\Sigma, G^{\prime}\right)$ corresponding to any homomorphism $h: G \rightarrow G^{\prime}$ of Abelian groups is the Cartesian product of the composition homomorphisms for the factor groups, with both $H^{1}(\Sigma, G)$ and $H^{1}\left(\Sigma, G^{\prime}\right)$ decomposed as in (i).

Given an Abelian group $G$, we denote by $G_{\text {ord2 }}$ the subgroup of $G$ consisting of zero and all elements of order 2. Thus,

$$
H^{1}\left(K^{2}, G\right)=G \times G_{\text {ord } 2},
$$

as one sees by combining Remark 25.2(i) with the relation $H_{1}\left(K^{2}, \mathbf{Z}\right)=\mathbf{Z} \times \mathbf{Z}_{2}$ at the beginning of this section. Obviously,

$$
\left(\mathbf{Z}_{q}\right)_{\text {ord2 }}=\{0, q / 2\} \text { for any even positive integer } q \text {. }
$$

Also, given closed real surfaces $\Sigma, \Sigma^{\prime}$ such that $\Sigma^{\prime}$ is orientable,

$$
\begin{aligned}
& \text { i) } H_{1}\left(\Sigma \# \Sigma^{\prime}, \mathbf{Z}\right)=H_{1}(\Sigma, \mathbf{Z}) \times H_{1}\left(\Sigma^{\prime}, \mathbf{Z}\right), \\
& \text { ii) } H^{1}\left(\Sigma \# \Sigma^{\prime}, G\right)=H^{1}(\Sigma, G) \times H^{1}\left(\Sigma^{\prime}, G\right), \\
& \text { iii) } \mathfrak{I}_{q}\left(\Sigma \# \Sigma^{\prime}\right)=\mathfrak{I}_{q}(\Sigma) \times \mathfrak{I}_{q}\left(\Sigma^{\prime}\right) \text { for } q \in\{2,4,6, \ldots, \infty\} \text {, }
\end{aligned}
$$

where $\mathfrak{I}_{q}(\Sigma)$ is defined by (2.9), with $\mathbf{Z}_{\infty}=\mathbf{Z}$. Namely, the well-known isomorphic identification (25.3) i) combined with Remark 25.2(i) and (1.5) gives (25.3) ii). Next, $w_{1}\left(\Sigma \# \Sigma^{\prime}\right)=\left(w_{1}(\Sigma), w_{1}\left(\Sigma^{\prime}\right)\right)$ (in terms of (25.3) ii) with $\left.G=\mathbf{Z}_{2}\right)$, since, by (1.6),$w_{1}\left(\Sigma \# \Sigma^{\prime}\right)$, acting on $H_{1}(\cdot, \mathbf{Z})$ rather than $\pi_{1}(\cdot)$, coincides with the first-factor projection in (25.3) i) followed by $w_{1}(\Sigma)$. Using Remark 25.2(ii) and (2.9), we now get (25.3) iii).

On the other hand, for the Klein bottle $K^{2}$ and $q \in\{2,4,6, \ldots, \infty\}$,

$$
\mathfrak{I}_{q}\left(K^{2}\right)=\left(\mathbf{Z}_{q} \backslash 2 \mathbf{Z}_{q}\right) \times\left[\left(\mathbf{Z}_{q}\right)_{\operatorname{ord} 2} \cap 2 \mathbf{Z}_{q}\right] .
$$

In fact, combining the description of $w_{1}\left(K^{2}\right)$ at the beginning of this section with (25.1) for $G=\mathbf{Z}_{2}$, we get $w_{1}\left(K^{2}\right)=(1,0) \in \mathbf{Z}_{2} \times \mathbf{Z}_{2}\left(\right.$ as $\left.\left(\mathbf{Z}_{2}\right)_{\text {ord } 2}=\mathbf{Z}_{2}\right)$. Now (25.4) is immediate from Remark 25.2(ii) and (25.1).

For a closed manifold $\Sigma$ and an even positive integer $q$, the $\bmod q$ reduction homomorphism $H^{1}(\Sigma, \mathbf{Z}) \rightarrow H^{1}\left(\Sigma, \mathbf{Z}_{q}\right)$ sends the set $\mathfrak{I}_{\infty}(\Sigma)$ into $\mathfrak{I}_{q}(\Sigma)$. (Notation of (2.9), with $\mathbf{Z}_{\infty}=\mathbf{Z}$.) This is clear from Remark 1.1] as $q$ is even, reduction $\bmod q$ followed by reduction mod 2 results in reduction $\bmod 2$. We thus obtain the $\bmod q$ reduction mapping

$$
\mathfrak{I}_{\infty}(\Sigma) \stackrel{\bmod q}{\longrightarrow} \mathfrak{I}_{q}(\Sigma) .
$$


Lemma 25.3. Given a closed real surface $\Sigma$ and an even integer $q>0$,

(i) the mapping (25.5) is surjective if and only if either $\Sigma$ is orientable, or $\Sigma$ is nonorientable and $\chi(\Sigma)-q / 2$ is odd,

(ii) if $q=4$ and $\Sigma$ is the Klein bottle $K^{2}$, the image of (25.5) is the 2-element subset $\{1,3\} \times\{0\}$ of the 4-element set

$$
\mathfrak{I}_{4}\left(K^{2}\right)=\{1,3\} \times\{0,2\},
$$

which itself is a subset of $H^{1}\left(K^{2}, \mathbf{Z}_{4}\right)=\{0,1,2,3\} \times\{0,2\} \subset \mathbf{Z}_{4} \times \mathbf{Z}_{4}$, cf. (25.1), with $\mathbf{Z}_{4}=\{0,1,2,3\}$.

Proof. Succesively applying (25.3) iii) we obtain, from (24.1) - (24.3) and (25.4), with superscripts denoting Cartesian powers, $\mathfrak{I}_{q}(\Sigma)=\left(2 \mathbf{Z}_{q}\right)^{2 g}$ in case (a) of Remark 25.1] $\mathfrak{I}_{q}(\Sigma)=\left(2 \mathbf{Z}_{q}\right)^{g-2} \times\left(\mathbf{Z}_{q} \backslash 2 \mathbf{Z}_{q}\right) \times\left[\left(\mathbf{Z}_{q}\right)_{\text {ord } 2} \cap 2 \mathbf{Z}_{q}\right]$ in case (b), and $\mathfrak{I}_{q}(\Sigma)=\left(2 \mathbf{Z}_{q}\right)^{g-1} \times\left[\left(\mathbf{Z}_{q}\right)_{\text {ord } 2} \backslash 2 \mathbf{Z}_{q}\right]$ in case (c) $\left(\right.$ as $\mathfrak{I}_{q}\left(\mathbf{R P}{ }^{n}\right)=\left(\mathbf{Z}_{q}\right)_{\operatorname{ord} 2} \backslash 2 \mathbf{Z}_{q}$ for $q \in\{2,4,6, \ldots, \infty\}$; cf. (24.3) and (25.2)). In view of Remark 25.2 (ii), the mapping (25.5) acts by $\bmod q$ reduction in each Cartesian factor set just listed. Also, since $q$ is even, the reduction mappings $\mathbf{Z} \rightarrow \mathbf{Z}_{q}$ and $\mathbf{Z} \backslash 2 \mathbf{Z} \rightarrow \mathbf{Z}_{q} \backslash 2 \mathbf{Z}_{q}$ are surjective. Therefore, surjectivity of (25.5) always holds in case (a) of Remark 25.1, while in case (b) or (c) it is equivalent to $\left(\mathbf{Z}_{q}\right)_{\text {ord } 2} \cap 2 \mathbf{Z}_{q}=\{0\}$ or, respectively, $\left(\mathbf{Z}_{q}\right)_{\text {ord } 2} \backslash 2 \mathbf{Z}_{q}=\varnothing$. Now (i) is immediate from (25.2), as $\chi(\Sigma)=2-g$ in cases (b) and (c). Finally, (25.1) - (25.2) give $H^{1}\left(K^{2}, \mathbf{Z}_{4}\right)=\{0,1,2,3\} \times\{0,2\}$, so that (25.6) follows from (25.4) for $q=4$, and (ii) is immediate as $\mathbf{Z}_{\text {ord } 2} \cap 2 \mathbf{Z}=\{0\}$.

\section{More on tori And Klein bottles in $\mathbf{C}^{2}$}

We will now evaluate the Maslov index $\boldsymbol{i}$ and degree $\boldsymbol{d}$ for the totally real immersions $f^{k, l}$ constructed in Example 20.2.

When $\Sigma$ is the torus $T^{2}$ or the Klein bottle $K^{2}$, (24.2) and (25.4) with $n=2$ and $q=\infty$ become $\mathfrak{I}_{\infty}\left(T^{2}\right)=2 \mathbf{Z} \times 2 \mathbf{Z} \subset \mathbf{Z} \times \mathbf{Z}=H^{1}\left(T^{2}, \mathbf{Z}\right)$ and $\mathfrak{I}_{\infty}\left(K^{2}\right)=$ $(\mathbf{Z} \backslash 2 \mathbf{Z}) \times\{0\} \subset \mathbf{Z} \times\{0\}=H^{1}\left(K^{2}, \mathbf{Z}\right)$ (cf. (25.1), (25.2) $)$.

A totally real immersion $(x, u): \mathbf{R}^{2} \rightarrow \mathbf{C}^{2}$ which is doubly $2 \pi$-periodic (or, in addition, also invariant under the transformation $\Phi$ of Example 20.1) descends to a totally real immersion $f: \Sigma \rightarrow \mathbf{C}^{2}$ with $\Sigma=T^{2}$ (or, respectively, $\Sigma=K^{2}$ ). Let us now set, for $\phi=\mathcal{J}(x, u)$ defined as in Example 9.1,

$$
p=\frac{1}{2 \pi i} \int_{0}^{2 \pi} \frac{\phi_{s}}{\phi} d s, \quad r=\frac{1}{2 \pi i} \int_{0}^{2 \pi} \frac{\phi_{t}}{\phi} d t, \quad m=\frac{1}{\pi i} \int_{0}^{\pi}\left[\frac{\phi_{t}}{\phi}\right]_{s=0} d t
$$

where the subscripts represent partial derivatives. Thus, $p, r, m \in \mathbf{Z}$ and the Maslov index of $f$ (see Section 2) is

$$
\boldsymbol{i}(f)= \begin{cases}(2 p, 2 r) \in 2 \mathbf{Z} \times 2 \mathbf{Z}=\mathfrak{I}_{\infty}(\Sigma) & \text { if } \quad \Sigma=T^{2}, \\ (m, 0) \in(\mathbf{Z} \backslash 2 \mathbf{Z}) \times\{0\}=\mathfrak{I}_{\infty}\left(K^{2}\right) & \text { if } \quad \Sigma=K^{2} .\end{cases}
$$

The following two propositions are easy consequences of the preceding discussion.

Proposition 26.1. Let $\Sigma$ stand for the 2-torus $T^{2}$ or the Klein bottle $K^{2}$, and let $f: \Sigma \rightarrow \mathbf{C}^{2}$ be a totally real immersion obtained as in Example 20.1 from some 
$x(t, s), y(t, s)$ and $h(t)$ satisfying the hypotheses of Example 20.1. Then $f$ has the Maslov index (26.2) with

$$
\begin{aligned}
& p=(2 \pi i)^{-1} \int_{0}^{2 \pi}\left(x_{s s} / x_{s}\right) d s, \\
& r=(2 \pi i)^{-1} \int_{0}^{2 \pi}\left[\left(x_{s t} / x_{s}\right)+\left(h_{t t} / h_{t}\right)\right] d t, \text { and } \\
& m=(\pi i)^{-1} \int_{0}^{\pi}\left[\left(x_{s t} / x_{s}\right)+\left(h_{t t} / h_{t}\right)\right]_{s=0} d t,
\end{aligned}
$$

the subscripts being partial derivatives.

In fact, (26.1) holds for $\phi=\phi^{[a]}$ with $\phi^{[a]}=a^{-1} \mathcal{J}(x, y+a h)$ and large $a>0$ (notation of Example 9.1). Homotopy invariance of the degree then guarantees that the integrals (26.1) do not depend on $a$. Taking their limits as $a \rightarrow \infty$, we obtain our assertion, since $\lim _{a \rightarrow \infty} \phi^{[a]}=x_{s} h_{t}$ by (20.1).

Proposition 26.2. There exist totally real embeddings of the 2-torus $T^{2}$ and the Klein bottle $K^{2}$ in $\mathbf{C}^{2}$ which realize any prescribed Maslov index $\mathbf{i}(f)$ with (26.2).

Proof. To exhibit such embeddings, we first apply Proposition 26.1 to the totally real embeddings $f^{k, 1}: T^{2} \rightarrow \mathbf{C}^{2}$ for $k \in \mathbf{Z}$ and $f^{k, 2}: K^{2} \rightarrow \mathbf{C}^{2}$ for odd $k$, described in Example 20.2 The mapping $F: \mathbf{R} / 2 \pi \mathbf{Z} \rightarrow S^{1}$ defined by the assignment $\mathbf{R} \ni s \mapsto x_{s} /\left|x_{s}\right|$ (with any fixed $t$ ) now is of degree zero (since $x_{s}(t, s+\pi)=-\overline{x_{s}(t, s)}$, and so $F$ is homotopic to its composite with the conjugation). Therefore, by Proposition 26.1, we have $p=0$; that is, $f^{k, 1}$ and $f^{k, 2}$ have the Maslov indices $(2 p, 2 r)=(0,2 k+2)$ and, respectively, $(m, 0)=(k+2,0)$. We thus have realized all index values for the Klein bottle. To obtain an arbitrary Maslov index $(2 p, 2 r)$ for the torus, we set $k=-1$ (when $p=r=0$ ) or, when $(p, r) \neq(0,0)$, use the composite of our $f$, for $k=d-1$, with the group automorphism of $T^{2}$ corresponding to a suitable matrix $\mathfrak{A} \in \mathrm{SL}(2, \mathbf{Z})$. Specifically, we fix $b, c \in \mathbf{Z}$ such that $b r-c p=d$, where $d$ is the greatest common factor of $p$ and $r$, and then choose $\mathfrak{A}$ to have the rows $\left[\begin{array}{ll}b & c\end{array}\right]$ and $[p / d \quad r / d]$.

\section{The INTEGER $q$ IN (1.4)}

For a simply connected almost complex manifold $M$, let $q$ be as in (1.4).

(a) $w_{2}(M)$ is the mod 2 reduction of $c_{1}(M)$.

(b) The connecting homomorphism in (1.1) for $E=E^{+}(M)$ is, up to a sign, the composite $\pi_{2} M \rightarrow H_{2}(M, \mathbf{Z}) \rightarrow \mathbf{Z}$ of the Hurewicz isomorphism with $c_{1}(M)$, and so its image, that is, the kernel of $\mathbf{Z} \rightarrow \pi_{1} E$ in (1.1), is trivial if $q=\infty$, and generated by $q / 2$ if $q<\infty$.

(c) $M$ is spin if and only if $q=\infty$ or $q$ is finite and divisible by 4 . Similarly, $q=\infty$ if and only if $c_{1}(M)=0$.

(d) If $M$ is not spin, or $c_{1}(M)=0$, the $\bmod 2$ reduction homomorphism $H_{2}(M, \mathbf{Z}) \rightarrow H_{2}\left(M, \mathbf{Z}_{2}\right)$ maps $\operatorname{Ker}\left[c_{1}(M)\right]$ onto $\operatorname{Ker}\left[w_{2}(M)\right]$.

(e) If $M=\mathbf{C}^{2}$, or $M=\mathbf{C P}^{2}$, or $M=\mathbf{C P}^{1} \times \mathbf{C P}^{1}$, then $q=\infty$, or $q=6$, or, respectively, $q=4$.

(f) $q=2$ whenever $M$ is obtained by blowing up a point in a simply connected complex surface.

In fact, (a) and (b) are well known, while (c) follows from (a), (b) and Remark 1.1. As $H_{2}(M, \mathbf{Z}) \rightarrow H_{2}\left(M, \mathbf{Z}_{2}\right)$ is surjective, (a) yields (d) in the case where $c_{1}(M)=$ 0 (cf. Remark 1.1). Again by (a), if $M$ is not spin, $c_{1}(M): H_{2}(M, \mathbf{Z}) \rightarrow \mathbf{Z}$ must assume some odd values; the homomorphism sending $H_{2}(M, \mathbf{Z}) / \operatorname{Ker}\left[c_{1}(M)\right] \approx \mathbf{Z}$ into $H_{2}\left(M, \mathbf{Z}_{2}\right) / \operatorname{Ker}\left[w_{2}(M)\right] \approx \mathbf{Z}_{2}$, induced by the $\bmod 2$ reduction, is therefore 
surjective. Every element of $\operatorname{Ker}\left[w_{2}(M)\right]$ thus has a preimage in $H_{2}(M, \mathbf{Z})$ with an even image in $H_{2}(M, \mathbf{Z}) / \operatorname{Ker}\left[c_{1}(M)\right]$, which proves (d). Next, (a) implies (e), as the values assigned by $c_{1}(M)$ to the standard generator(s) of $H_{2}(M, \mathbf{Z})$ for $M=\mathbf{C P}^{2}$ or $M=\mathbf{C P}^{1} \times \mathbf{C P}^{1}$ are 3 or, respectively, 2 and 2 . Finally, (f) follows from (a), since $c_{1}(M)$ yields the value 1 when integrated over the exceptional divisor resulting from the blow-up (cf. also formula (30.1)).

\section{INDEX AND DEGREE AFTER MODIFICATIONS}

We now determine how the zooming and connected-sum operations of Sections 10 and 18 affect the Maslov index and degree.

Lemma 28.1. Given a totally real immersion/embedding $f$ of a closed real surface $\Sigma$ in $\mathbf{C}^{2}$, and a simply connected almost complex surface $M$, let a totally real immersion/embedding $f^{\prime}: \Sigma \rightarrow M$ be obtained from $f$ by a zooming procedure described in Proposition 10.1. Its Maslov index and degree, introduced in Section 2 , then are

$$
\boldsymbol{i}\left(f^{\prime}\right)=[\boldsymbol{i}(f) \bmod q] \in \mathfrak{I}_{q}(\Sigma) \subset H^{1}\left(\Sigma, \mathbf{Z}_{q}\right), \quad \boldsymbol{d}\left(f^{\prime}\right)=0,
$$

where $q$ is determined by $M$ via (1.4) and $[\boldsymbol{i}(f) \bmod q]$ denotes the $\bmod q$ reduction of $\mathbf{i}(f) \in H^{1}(\Sigma, \mathbf{Z})$, that is, its image under the mapping (25.5).

Proof. Let $U \subset \mathbf{C}^{2}$ be a ball containing $f(\Sigma)$. Since $U$ is contractible, $\boldsymbol{d}\left(f^{\prime}\right)=0$. Also, $\boldsymbol{i}\left(f^{\prime}\right)$ is the homomorphism (3.3) of fundamental groups induced by $\mathfrak{M}\left(f^{\prime}\right)$. Thus, $\boldsymbol{i}\left(f^{\prime}\right)$ equals $\boldsymbol{i}(f)$ followed by the homomorphism $\pi_{1}[E(U)] \rightarrow \pi_{1}[E(M)]$ induced by an embedding $U \rightarrow M$. As the fibre inclusion $\mathrm{U}(1) \rightarrow E(M)$ represents a generator of $\pi_{1}[E(M)$, the latter homomorphism is nothing other than the projection $\mathbf{Z} \rightarrow \mathbf{Z}_{q}$.

Lemma 28.1 can be modified when $M$ is a complex surface. Rather than by zooming, $f^{\prime}$ may then be obtained as the composite of $f$ with a holomorphic embedding in $M$ of an open ball in $\mathbf{C}^{2}$ containing $f(\Sigma)$.

The following corollary is an immediate consequence of Lemma 28.1 Proposition 26.2 and Lemma 25.3. We use it later to prove Theorems 28.6, 2.16 and 2.17. Proposition 29.1] and the claim made in Example 36.1.

Corollary 28.2. Given a simply connected almost complex surface $M$, let us consider the following condition imposed on a closed real surface $\Sigma$ and an element $\boldsymbol{i} \in \mathfrak{I}_{q}(\Sigma)$, with $q$ and $\mathfrak{I}_{q}(\Sigma)$ defined by (1.4) and (2.9):

(*) some totally real embedding $f: \Sigma \rightarrow M$ has $\boldsymbol{i}(f)=\boldsymbol{i}$ and $\boldsymbol{d}(f)=0$.

Condition $(*)$ is satisfied by

(a) the torus $\Sigma=T^{2}$ and every $\boldsymbol{i} \in \mathfrak{I}_{q}(\Sigma)$,

(b) the Klein bottle $\Sigma=K^{2}$ and every $\mathbf{i} \in \mathfrak{I}_{q}(\Sigma)$, provided that $q / 2$ is either infinite, or finite and odd,

(c) the Klein bottle $\Sigma=K^{2}$ and every $\boldsymbol{i}$ in the subset $\{1,3\} \times\{0\}$ of $\mathfrak{I}_{4}(\Sigma)$, cf. (25.6), provided that $q=4$.

Lemma 28.3. Let $f, f^{\prime}$ be totally real immersions of closed real surfaces $\Sigma, \Sigma^{\prime}$ in a simply connected almost complex surface $M$. If $\Sigma^{\prime}$ is orientable, then a totally real immersion $\Sigma \# \Sigma^{\prime} \rightarrow M$ of the connected sum, obtained from $f$ and $f^{\prime}$ as in Theorem 18.1, has the Maslov index and degree

$$
\boldsymbol{i}=\left(\mathbf{i}(f), \mathbf{i}\left(f^{\prime}\right)\right), \quad \boldsymbol{d}=\boldsymbol{d}(f)+\boldsymbol{d}\left(f^{\prime}\right) \in H_{2}\left(M, \mathbf{Z}_{[2]}\right) .
$$


Here we use the notational conventions of (25.3) iii) and (2.3), while adding an element of $H_{2}(M, \mathbf{Z})$ to an element of $H_{2}\left(M, \mathbf{Z}_{2}\right)$ is to be preceded by $\bmod 2$ reduction of the former, so that the sum lies in $H_{2}\left(M, \mathbf{Z}_{2}\right)$.

In fact, (28.2) follows from (25.3) i), since the new immersion coincides with $f$ or $f^{\prime}$ except on the boundary of a solid cylinder immersed in $M$ (Theorem 18.1(v)).

Next, for $\mathcal{Z}(\Sigma, M)$ defined in the lines following Theorem 2.2 we have

Lemma 28.4. Given a simply connected almost complex surface $M$, let $\mathcal{E}$ be the class of diffeomorphism types of closed real surfaces $\Sigma$ such that for every $(\boldsymbol{i}, \boldsymbol{d}) \in \mathcal{Z}(\Sigma, M)$ there exists a totally real immersion $f: \Sigma \rightarrow M$ with $\boldsymbol{i}(f)=\boldsymbol{i}$ and $\boldsymbol{d}(f)=\boldsymbol{d}$. Then $\mathcal{E}$ is closed under the connected-sum operation applied to two surfaces, of which at least one is orientable.

Proof. Let $(\boldsymbol{i}, \boldsymbol{d}) \in \mathcal{Z}\left(\Sigma \# \Sigma^{\prime}, M\right)$, where $\Sigma, \Sigma^{\prime}$ belong to $\mathcal{E}$ and $\Sigma^{\prime}$ is orientable. By (25.3) iii), $\boldsymbol{i}=\left(\mathbf{i}_{0}, \mathbf{i}^{\prime}\right)$ with $\boldsymbol{i}_{0} \in \mathfrak{I}_{q}(\Sigma)$ and $\boldsymbol{i}^{\prime} \in \mathfrak{I}_{q}\left(\Sigma^{\prime}\right)$, for $q$ as in (1.4), so that $\left(\mathbf{i}_{0}, \boldsymbol{d}\right) \in \mathcal{Z}(\Sigma, M)$ due to the definition of $\mathcal{Z}(\Sigma, M)$. Similarly, $\left(\boldsymbol{i}^{\prime}, 0\right)$ is an element of $\mathfrak{I}_{q}\left(\Sigma^{\prime}\right) \times\{0\} \subset \mathfrak{I}_{q}\left(\Sigma^{\prime}\right) \times \mathfrak{D}_{+}^{1}(M)=\mathcal{Z}\left(\Sigma^{\prime}, M\right)$. Thus, $\left(\boldsymbol{i}_{0}, \boldsymbol{d}\right)$ and $\left(\boldsymbol{i}^{\prime}, 0\right)$ are realized as the index-degree pairs of some totally real immersions $\Sigma \rightarrow M$ and $\Sigma^{\prime} \rightarrow M$. Realizability of $(\boldsymbol{i}, \boldsymbol{d})$ now follows from Lemma 28.3

For a simply connected almost complex surface $M$, consider the condition

$q / 2$ is infinite, or finite and even, or, finally, $\mathbf{R P}^{2}$ admits a totally real immersion in $M$, while $q / 2$ is finite and odd,

with $q$ defined by (1.4). Further conditions imposed on $M$ are:

totally real immersions $S^{2} \rightarrow M$ realize every degree $\boldsymbol{d}$ in some set generating the group $\operatorname{Ker}\left[c_{1}(M)\right] \subset H_{2}(M, \mathbf{Z})$

$\left(\varnothing\right.$ is such a set when $\left.\operatorname{Ker}\left[c_{1}(M)\right]=\{0\}\right)$, and, for a closed real surface $\Sigma$,

every $\boldsymbol{i} \in \mathfrak{I}_{q}(\Sigma)$, with $q$ as in (1.4), equals the Maslov index $\boldsymbol{i}(f)$ of some totally real immersion $f: \Sigma \rightarrow M$.

Here we use the notation of Section 2 ,

Lemma 28.5. Suppose that $\Sigma$ is a closed real surface and $M$ is a simply connected almost complex surface such that either $M$ is not spin, or $c_{1}(M)=0$. If conditions (28.4) and (28.5) are satisfied, then $\Sigma$ belongs to the class $\mathcal{E}$ defined in Lemma 28.4 .

Proof. Let $(\boldsymbol{i}, \boldsymbol{d}) \in \mathcal{Z}(\Sigma, M)=\mathfrak{I}_{q}(\Sigma) \times \mathfrak{D}_{ \pm}^{\varepsilon}(M)$. (Cf. (c) in Section 27 and the definition of $\mathcal{Z}(\Sigma, M)$ in the lines following Theorem 2.2.) Choose $f$ for this $\boldsymbol{i}$ as in (28.5). As $\boldsymbol{d}, \boldsymbol{d}(f) \in \mathfrak{D}_{ \pm}^{\varepsilon}(M)$ (Lemma 7.1(b)), the difference $\boldsymbol{d}^{\prime}=\boldsymbol{d}-\boldsymbol{d}(f)$ lies in $\operatorname{Ker}\left[c_{1}(M)\right]$ (if $\Sigma$ is orientable) or in $\operatorname{Ker}\left[w_{2}(M)\right]$ (if $\Sigma$ is not orientable). Thus, even in the nonorientable case, $\boldsymbol{d}^{\prime}$ is the $\bmod 2$ reduction of an element of $\operatorname{Ker}\left[c_{1}(M)\right]$ (see (d) in Section 27).

Hence, in view of (28.4) combined with Lemma 28.3 and Corollary 11.3. $(\boldsymbol{i}, \boldsymbol{d})$ is the index-degree pair of a totally real immersion $\Sigma \rightarrow M$.

The following result, proved below by a direct elementary argument, can also be derived from the $h$-principle (via Theorem 2.2). 
Theorem 28.6. Let a simply connected almost complex surface $M$ satisfy conditions (28.3) and (28.4). If the class $\mathcal{E}$ defined in Lemma 28.4 contains the Klein bottle $\Sigma=K^{2}$, then $\mathcal{E}$ contains every closed real surface.

Proof. Every closed surface either is one of $S^{2}, T^{2}, \mathbf{R} P^{2}, K^{2}$, or can be obtained by iterated connected summation in which all intermediate summands, except (possibly) the last one, are orientable. (Cf. Remark 25.1.) Thus, by Lemma 28.4, it suffices to show that $\mathcal{E}$ contains $S^{2}, T^{2}$ and $\mathbf{R} \mathbf{P}^{2}$. This will in turn follow from Lemma 28.5, once we show that condition (28.5) holds when $\Sigma$ is any of these three real surfaces.

First, (28.5) is obvious for $\Sigma=\mathbf{R P}^{2}$, since, by (24.3), $\mathfrak{I}_{q}\left(\mathbf{R P} \mathbf{P}^{2}\right)$ is a one-element set when $q / 2$ is finite and odd, and empty otherwise. Similarly, (28.5) for $\Sigma=S^{2}$ is immediate since $\mathfrak{I}_{q}\left(S^{2}\right)$ always has just one element (see (24.1)), and a totally real immersion $S^{2} \rightarrow M$ exists by Corollary 11.3. Finally, (28.5) for $\Sigma=T^{2}$ is obvious from Corollary 28.2(a).

\section{Totally Real Klein bottles in $\mathbf{C P}^{1} \times \mathbf{C P}^{1}$}

Let $M=\mathbf{C P}^{1} \times \mathbf{C P}^{1}$. The standard isomorphic identification

$$
H_{2}\left(\mathbf{C P}^{1} \times \mathbf{C P}^{1}, \mathbf{Z}_{[2]}\right)=\mathbf{Z}_{[2]} \times \mathbf{Z}_{[2]}, \quad \text { with } \mathbf{Z}_{[2]} \text { as in (2.3), }
$$

in which the generators $(1,0),(0,1)$ of $\mathbf{Z}_{[2]} \times \mathbf{Z}_{[2]}$ correspond to cycles of the form $\mathbf{C P}^{1} \times\{y\}$ and $\{x\} \times \mathbf{C P}^{1}$, gives

$$
(a, b) \cdot\left(a^{\prime}, b^{\prime}\right)=a b^{\prime}+b a^{\prime} \text { for } a, b, a^{\prime}, b^{\prime} \in \mathbf{Z}_{[2]},
$$

with $\cdot$ denoting the $\mathbf{Z}_{[2]}$-valued intersection form in $H_{2}\left(M, \mathbf{Z}_{[2]}\right)$. In terms of the identification (29.1),

$$
\operatorname{Ker}\left[c_{1}(M)\right]=\{(a, b) \in \mathbf{Z} \times \mathbf{Z}: a+b=0\} \subset H_{2}(M, \mathbf{Z}) .
$$

In fact, $c_{1}(M)$ treated as a homomorphism $\mathbf{Z} \times \mathbf{Z}=H_{2}(M, \mathbf{Z}) \rightarrow \mathbf{Z}$ acts by $(a, b) \mapsto 2(a+b)$, since it sends both $(1,0)$ and $(0,1)$ to 2 .

For the Klein bottle $\Sigma=K^{2}$ and $M=\mathbf{C P}^{1} \times \mathbf{C P}^{1}$, one now easily sees that $[\{(1,0),(3,0)\} \times\{(0,0),(1,1)\}] \cup[\{(1,2),(3,2)\} \times\{(1,0),(0,1)\}]$ is the set $\mathcal{Z}(\Sigma, M)$ defined in the lines following Theorem 2.2. (This is clear from (25.6) with $H_{1}\left(K^{2}, \mathbf{Z}\right)=\mathbf{Z} \times \mathbf{Z}_{2}$; cf. Section 25, and (29.3).) Thus, $\mathcal{Z}(\Sigma, M)$ is an 8-element subset of the 16 -element set $\mathfrak{I}_{4}(\Sigma) \times \mathfrak{D}_{+}^{0}(M)$. Here $\mathfrak{I}_{4}(\Sigma)=\{1,3\} \times\{0,2\}$ by (25.6), $q=4\left((\mathrm{e})\right.$ in Section 27), and $\mathfrak{D}_{+}^{0}(M)=H_{2}\left(M, \mathbf{Z}_{2}\right)=\{0,1\} \times\{0,1\}$ for $M=\mathbf{C P}^{1} \times \mathbf{C P}^{1}$, by (2.8).

The following result will be proved at the end of this section.

Proposition 29.1. Let $M=\mathbf{C P}^{1} \times \mathbf{C P}^{1}$ and $\Sigma=K^{2}$. Every element $(\boldsymbol{i}, \boldsymbol{d})$ of $\mathcal{Z}(\Sigma, M)$ then equals $(\mathbf{i}(f), \boldsymbol{d}(f))$ for some totally real immersion $f: \Sigma \rightarrow M$. If, in addition, $\boldsymbol{d} \neq(1,1)$, then $(\boldsymbol{i}, \boldsymbol{d})=(\boldsymbol{i}(f), \boldsymbol{d}(f))$ for some totally real embedding $f: \Sigma \rightarrow M$.

Remark 29.2. An anti-diagonal totally real 2-sphere $\Sigma \subset \mathbf{C P}^{1} \times \mathbf{C P}^{1}$ (see (vii) in Section 9), suitably oriented, has the degree $\boldsymbol{d}=(1,-1) \in \mathbf{Z} \times \mathbf{Z}$; cf. (29.1). In fact, writing $\boldsymbol{d}=(a, b)$ we get $b=\mp 1$, since the composite of the mapping $x \mapsto(\bar{x}, x)$ followed by the projection onto the second factor is $\mathrm{Id}: \mathbf{C P}^{1} \rightarrow \mathbf{C P}^{1}$. Therefore, $a= \pm 1$ due to relations (5.3) b) and (29.3). 
Remark 29.3. Given a totally real immersion $f: \Sigma \rightarrow M$ of a closed real surface $\Sigma$ in a simply connected almost complex surface $M$ and a diffeomorphism $h: M \rightarrow M$ preserving the almost complex structure, we have $\boldsymbol{i}(h \circ f)=\boldsymbol{i}(f)$. The reason is that $h$, when naturally lifted to $E(M)$, acts trivially on $\pi_{1}[E(M)]$, as it commutes with the $\mathrm{U}(1)$ action which provides a generator of $\pi_{1}[E(M)]$.

The next lemma is similar to a result of Stout and Zame [27, Theorem 1], who prove, given compact manifolds $\Sigma$ and $\Sigma^{\prime}$ of dimensions $n$ and $k$, with $\chi(\Sigma)=0$, admitting totally real immersions in $\mathbf{C}^{n}$ and, respectively, $\mathbf{C}^{k}$, that the product manifold $\Sigma \times \Sigma^{\prime}$ must admit a totally real embedding in $\mathbf{C}^{n+k}$.

Lemma 29.4. Suppose that $N, P$ are almost complex manifolds, $\Sigma$ is the total space, $\operatorname{pr}: \Sigma \rightarrow Q$ is the projection of a locally trivial fibre bundle over a real manifold $Q$, and $h: Q \rightarrow N$ is a totally real immersion/embedding. Moreover, let $\Phi: \Sigma \rightarrow P$ be a $C^{\infty}$ mapping whose restriction to each fibre $\Sigma_{y}, y \in Q$, is a totally real immersion/embedding. Then $f$ given by $f(x)=(h(\operatorname{pr}(x)), \Phi(x))$, for $x \in \Sigma$, is a totally real immersion/embedding of $\Sigma$ in the product almost complex manifold $M=N \times P$.

In fact, nonzero horizontal and vertical vectors in $\Sigma$ have $d f$-images that are linearly independent over $\mathbf{C}$.

Proof of Proposition 29.1. Again, let $M=\mathbf{C P}^{1} \times \mathbf{C P}^{1}$ and $\Sigma=K^{2}$. It suffices to show that every $\boldsymbol{d}=(a, b) \in\{0,1\} \times\{0,1\}=\mathbf{Z}_{2} \times \mathbf{Z}_{2}=H_{2}\left(M, \mathbf{Z}_{2}\right)$ is realized as the degree $\boldsymbol{d}(f)$ of some totally real immersion $f: \Sigma \rightarrow M$, injective if $\boldsymbol{d} \neq(1,1)$, and having the Maslov index $\mathbf{i}(f) \in \mathfrak{I}_{4}(\Sigma)=\{1,3\} \times\{0,2\}$ with the required second component $2|a-b|$. Namely, when $f$ is replaced by its composite $f^{\prime}$ with the diffeomorphism $K^{2} \rightarrow K^{2}$ described immediately before Remark 25.1, the possible values 1,3 of the first component of $\boldsymbol{i}(f)$ become interchanged, while the second component and the degree remain the same. (In fact, that diffeomorphism acts by $(a, b) \mapsto(-a, b)$ in $H^{1}\left(K^{2}, G\right)=G \times G_{\text {ord2 }}$, cf. (25.1), for any Abelian group $G$, due to the way it operates on $H_{1}\left(K^{2}, \mathbf{Z}\right)$, as described before Remark 25.1. On the other hand, 3 is the opposite of 1 in $\mathbf{Z}_{4}$.)

First, Corollary 28.2(c) and Remark 29.2 allow us to choose totally real embeddings $f: \Sigma \rightarrow M$ and $f^{\prime}: S^{2} \rightarrow M$ with $\boldsymbol{d}(f)=(0,0)$ (proving realizability of $\boldsymbol{d}=(0,0))$ and $\boldsymbol{d}\left(f^{\prime}\right)=(1,-1) \in \mathbf{Z} \times \mathbf{Z}$. Lemma 28.3 now provides a totally real immersion $\Sigma=\Sigma \# S^{2} \rightarrow M$ with the degree $\boldsymbol{d}=(1,1) \in \mathbf{Z}_{2} \times \mathbf{Z}_{2}$ and the Maslov index $\boldsymbol{i}=\boldsymbol{i}(f) \in \mathfrak{I}_{4}(\Sigma)$ (where $\boldsymbol{i}\left(f^{\prime}\right)=0$ by (24.1), and we identify $\mathfrak{I}_{4}(\Sigma) \times\{0\}$ with $\left.\mathfrak{I}_{4}(\Sigma)\right)$. Thus, $\boldsymbol{d}=(1,1)$ is realized as well.

Next, to realize the degree $\boldsymbol{d}=(0,1)$, let us identify $\mathbf{C P}^{1}$ with the unit sphere $S^{2}$ centered at zero in $\mathbf{R}^{3}$, and define a totally real embedding $f: \Sigma \rightarrow M$ as in Lemma 29.4. choosing the base $Q$ to be any circle embedded in $\mathbf{C P}^{1}$ and the fibres to be the family of all great circles in $S^{2}=\mathrm{CP}^{1}$ that contain a fixed pair of antipodal points $u,-u \in S^{2}$. As a point $y$ traverses the base circle $Q$, we require the corresponding fibre $\Sigma_{y}$ to vary by being rotated about the axis $\mathbf{R} u$ in $\mathbf{R}^{3}$ in such a way that, after $y$ has gone all the way around $Q$, the fibre circle will have undergone a rotation by the angle $\pi$. That the resulting embedding $f: K^{2} \rightarrow M=\mathbf{C P}^{1} \times \mathbf{C P}^{1}$ is totally real follows from Lemma 29.4 (the totalreality assumptions being satisfied for dimensional reasons; cf. (i) in Section (9). That $\boldsymbol{d}(f)=(0,1)$ is in turn clear if one considers the images of $\boldsymbol{d}(f)$ under the factor projections: the projection of $f$ onto the first $\mathbf{C P}^{1}$ factor is not surjective, 
while $f$ projected onto the second factor is the blow-down projection $K^{2} \rightarrow \mathbf{C P}^{1}$ (with $K^{2}$ treated as a two-point real blow-up of $\mathrm{CP}^{1}=S^{2}$ ).

Finally, for $f$ chosen as above, with $\boldsymbol{d}(f)=(0,1)$, let $f^{\prime}$ be the composite of $f$ with the switch mapping $M \ni(y, z) \mapsto(z, y) \in M$. The totally real embedding $f^{\prime}: \Sigma \rightarrow M$ obviously has $\boldsymbol{d}\left(f^{\prime}\right)=(1,0)$. As $\boldsymbol{i}\left(f^{\prime}\right)=\boldsymbol{i}(f)$ (see Remark 29.3), this completes the proof.

\section{Surfaces immersed in $\mathbf{C P}^{2} \# m \overline{\mathbf{C P}^{2}}$}

For $M, M^{\prime}$ as in (2.11) and $c_{1}(\cdot): H_{2}(\cdot, \mathbf{Z}) \rightarrow \mathbf{Z}$, we have

$$
\left[c_{1}\left(M^{\prime}\right)\right]\left(\xi, q_{1}, \ldots, q_{m}\right)=\left[c_{1}(M)\right](\xi)-q_{1}-\ldots-q_{m}
$$

whenever $\xi \in H_{2}(M, \mathbf{Z})$ and $q_{1}, \ldots, q_{m} \in \mathbf{Z}$. In fact, for the tangent bundle $\tau$ and normal bundle $\nu$ of any of the $m$ exceptional divisors $\Sigma$ in $M^{\prime}$ resulting from the blow-up, $\left[c_{1}\left(M^{\prime}\right)\right]([\Sigma])=c_{1}(\tau)+c_{1}(\nu)=2+c_{1}(\nu)$ and $c_{1}(\nu)=[\Sigma] \cdot[\Sigma]=-1$. (Note the sign convention used in (2.11).)

We now discuss a special case, using further notational conventions. First, $M=$ $\mathbf{C P}^{2} \# m \overline{\mathbf{C P}^{2}}$ stands, in the rest of this section, for the complex surface obtained by blowing up any ordered set of $m \geq 1$ distinct points in $\mathbf{C P}^{2}$, while $\langle$,$\rangle and \mathbf{Z}^{m}$ are the standard Euclidean inner product of $\mathbf{R}^{m}$ and the additive subgroup of $\mathbf{R}^{m}$ generated by the standard basis $\mathbf{e}_{1}, \ldots, \mathbf{e}_{m}$. The identification (2.11) now yields

$$
H_{2}\left(\mathbf{C P}^{2} \# m \overline{\mathbf{C P}^{2}}, \mathbf{Z}\right)=\mathbf{Z}^{m+1}=\mathbf{Z} \times \mathbf{Z}^{m} \subset \mathbf{Z} \times \mathbf{R}^{m} .
$$

Specifically, the homology classes which correspond here to $(1, \mathbf{0}) \in \mathbf{Z} \times \mathbf{R}^{m}$ and $\left(0, \mathbf{e}_{j}\right) \in \mathbf{Z} \times \mathbf{R}^{m}, j=1, \ldots, m$, are realized by a projective line in $\mathbf{C P}^{2}$ not containing any of the blown-up points (with its standard orientation, described at the beginning of Section 5) and, respectively, by the $m$ embedded copies of $\mathbf{C P}^{1}$ that replace the blown-up points, each of them with the opposite of its standard orientation. Therefore,

$$
(d, \boldsymbol{q}) \cdot\left(d^{\prime}, \boldsymbol{q}^{\prime}\right)=d d^{\prime}-\left\langle\boldsymbol{q}, \boldsymbol{q}^{\prime}\right\rangle
$$

for the intersection form - in $H_{2}(M, \mathbf{Z})$, while $c_{1}(M): H_{2}(M, \mathbf{Z}) \rightarrow \mathbf{Z}$ acts by $(d, \boldsymbol{q}) \mapsto 3 d-q_{1}-\ldots-q_{m}$, where $\boldsymbol{q}=\left(q_{1}, \ldots, q_{m}\right)$ (see (30.1)). Thus,

$$
\operatorname{Ker}\left[c_{1}(M)\right]=\left\{\left(d, q_{1}, \ldots, q_{m}\right): q_{1}+\ldots+q_{m}=3 d\right\} \subset H_{2}(M, \mathbf{Z}) .
$$

Example 30.1. The totally real immersion $f: S^{2} \rightarrow \mathbf{C P}^{2} \# \overline{\mathbf{C P}^{2}}$ described in Corollary 15.3 for $m=1$ has $\boldsymbol{d}(f)=(1,3)$. In fact, let $\boldsymbol{d}(f)=(d, \boldsymbol{q})$. Then $d=1$ due to the intersection equality in Corollary 15.3 and (30.3) for $\left(d^{\prime}, \boldsymbol{q}^{\prime}\right)=(1, \mathbf{0})$, so that (5.3) b) and (30.4) for $m=1$ give $(d, \boldsymbol{q})=(1,3)$.

Example 30.2. A totally real embedded 2-sphere $\Sigma \subset \mathbf{C P}^{2} \# 2 \overline{\mathbf{C P}^{2}}$ with the degree $[\Sigma]=(0,1,-1)$, for a suitable orientation of $\Sigma$, can be obtained as follows. Using a complex automorphism of $\mathbf{C P}^{2}$, we may assume that the blown-up points are $x^{ \pm}=(0, \pm a) \in \mathbf{C} \times \mathbf{R} \subset \mathbf{C}^{2} \subset \mathbf{C P}^{2}$, with $a>0$. The 2 -sphere $S$ of radius $a$ in $\mathbf{C} \times \mathbf{R}$, centered at $(0,0)$, contains $x^{ \pm}$as its unique complex points, both removable by blow-up (Example 13.5). Blowing them up transforms $S$ into a totally real embedded sphere $\Sigma$ in $\mathbf{C P}^{2} \# 2 \overline{\mathbf{C P}^{2}}$. To see that $[\Sigma]=(0,1,-1)$, set $[\Sigma]=(d, \boldsymbol{q})$. Thus, $d=0$ from (30.3) applied to the homology class $\left(d^{\prime}, \boldsymbol{q}^{\prime}\right)=(1, \mathbf{0})$, represented by a projective line in $\mathbf{C P}^{2}$ not intersecting $S$. Next, (5.4) (for $n=2$ ) and (30.3) yield $\langle\boldsymbol{q}, \boldsymbol{q}\rangle=-(d, \boldsymbol{q}) \cdot(d, \boldsymbol{q})=\chi(\Sigma)=2$. The two components of $\boldsymbol{q}$ thus have the 
absolute value 1 , and opposite signs (by (5.3) b) combined with (30.4) for $d=0$ and $m=2$ ).

Remark 30.3. Condition (28.4) is satisfied by $M=\mathbf{C P}^{2} \# m \overline{\mathbf{C P}^{2}}, m \geq 1$. In fact, $\operatorname{Ker}\left[c_{1}(M)\right]$ is generated by $\left(1,3 \mathbf{e}_{1}\right)$ and $\left(0, \mathbf{e}_{j}-\mathbf{e}_{1}\right), j=2, \ldots, m$, with $\mathbf{e}_{j}$ as above. (See (30.4).) These generators are all realized by totally real immersions, in view of Examples 30.1, 30.2 and Lemma 12.1(a).

Proof of Theorem 2.16. We will verify below that the complex surfaces (0.1) all satisfy the assumptions, and hence conclusions, of Theorem 28.6. Thus, totally real immersions of closed real surfaces $\Sigma$ in the complex surfaces $M$ forming the list (0.1) realize, as their index-degree pairs, all elements $(\boldsymbol{i}, \boldsymbol{d})$ of the set $\mathcal{Z}(\Sigma, M) \subset \mathfrak{I}_{q}(\Sigma) \times \mathfrak{D}_{ \pm}^{\varepsilon}(M)$ described in the lines following Theorem 2.2. This will clearly imply Theorem 2.16, since in Section 7 we already proved that, conversely, all such index-degree pairs lie in $\mathcal{Z}(\Sigma, M)$.

First, (28.3) holds if $M$ is $\mathbf{C}^{2}, \mathbf{C P}^{1} \times \mathbf{C P}^{1}, \mathbf{C P}^{2}$, or $\mathbf{C P}^{2} \# m \overline{\mathbf{C P}}, m \geq 1$, as $q / 2$ then equals, respectively, $\infty, 2,3$ or 1 (see (e)-(f) in Section 27), and in the last two cases a totally real immersion $\mathbf{R P}^{2} \rightarrow M$ exists according to (vi) in Section 9 combined with Lemma 12.1(a).

Next, (28.4) is satisfied by $\mathbf{C}^{2}$ and $\mathbf{C P}^{2}$ in view of Corollary 11.3 (since $\operatorname{Ker}\left[c_{1}(\cdot)\right]$ is the trivial group); by $\mathbf{C P}^{1} \times \mathbf{C P}^{1}$, as a consequence of (29.3) and Remark 29.2, and by $\mathbf{C P}^{2} \# m \overline{\mathbf{C P}^{2}}, m \geq 1$, according to Remark 30.3

Finally, the assumption about $K^{2}$ in Theorem 28.6 holds for $\mathbf{C P}^{1} \times \mathbf{C P}^{1}$ by Proposition 29.1, and for the remaining complex surfaces (0.1) it is immediate from Lemma 28.5. 28.5) is satisfied, with $\Sigma=K^{2}$, by Corollary 28.2(b), since $q / 2 \in\{\infty, 3,1\}$ (as remarked above), while (28.4) was verified in the last paragraph. This completes the proof.

\section{Some special cases of Theorem 2.17}

Let $\Sigma$ and $M$ be, respectively, a fixed closed real surface and one of the complex surfaces (0.1). The pairs $(\boldsymbol{i}, \boldsymbol{d})$ that can be simultaneously realized as the Maslov index and degree of a totally real immersion $\Sigma \rightarrow M$ then are nothing other than all elements of the set $\mathcal{Z}(\Sigma, M)$. (See Theorem 2.16 and the discussion following it in Section 2.) This is, however, not the case if one replaces the word 'immersion' by 'embedding' since, in view of (5.4) and Corollary 23.2 (for $n=2$ ), the degree $\boldsymbol{d}=\boldsymbol{d}(f)$ of any totally real embedding $f: \Sigma \rightarrow M$ satisfies the additional constraint 2.10).

Theorem 2.17 states that there are no further constraints, as long as $m \leq 7$ in (0.1). In other words, the conditions $(\boldsymbol{i}, \boldsymbol{d}) \in \mathcal{Z}(\Sigma, M)$ and (2.10) hold if and only if the pair $(\boldsymbol{i}, \boldsymbol{d})$ is realized by a totally real embedding $\Sigma \rightarrow M$, where $M$ is $\mathbf{C}^{2}$, $\mathbf{C P}^{2}, \mathbf{C P}^{1} \times \mathbf{C P}^{1}$, or $\mathbf{C P}^{2} \# m \overline{\mathbf{C P}^{2}}, 1 \leq m \leq 7$.

In this section we establish Theorem 2.17 in the case where $\Sigma$ is orientable and $M$ is $\mathbf{C}^{2}, \mathbf{C P}^{2}$ or $\mathbf{C P}^{1} \times \mathbf{C P}^{1}$. (For the other cases, see Section 36]) Note that $\mathcal{Z}(\Sigma, M)=\mathfrak{I}_{q}(\Sigma) \times \operatorname{Ker}\left[c_{1}(M)\right]$ when $\Sigma$ is orientable, according to (2.7), (2.8) and the lines following Theorem 2.2 .

First, if $\Sigma$ is orientable and $M=\mathbf{C}^{2}$ or $M=\mathbf{C P}^{2}$, relations (24.4), (24.5) and (2.7) give $\boldsymbol{d} \in \operatorname{Ker}\left[c_{1}(M)\right]=\{0\}$, and so, by (2.10), $\Sigma$ must be the torus $T^{2}$. Our assertion is now obvious from Corollary 28.2(a). 
To verify Theorem 2.17 for orientable $\Sigma$ and $M=\mathbf{C P}^{1} \times \mathbf{C P}^{1}$, we use the notations of (29.1)-(29.2). In view of (29.2)-(29.3), conditions $\boldsymbol{d} \in \operatorname{Ker}\left[c_{1}(M)\right]$ and (2.10), for any given closed oriented surface $\Sigma$, now read $a+b=0$ and $2 a b=-\chi(\Sigma)$, where $\boldsymbol{d}=(a, b)$. This gives $\chi(\Sigma)=-2 a b=2 a^{2} \geq 0$; that is, $\Sigma$ can only be the torus $T^{2}$ with $a=b=0$ or the sphere $S^{2}$ with $(a, b)=( \pm 1, \mp 1)$. A totally real embedding $\Sigma \rightarrow M$ realizing the pair $(\boldsymbol{i}, \boldsymbol{d})$ for any $\mathbf{i} \in \mathfrak{I}_{4}(\Sigma)$ (cf. (e) in Section 27) and our $\boldsymbol{d}=(a, b)$ thus exists in view of Corollary 28.2(a) (for $\Sigma=T^{2}$ ) or Remark 29.2 (for $\Sigma=S^{2}$ ). Note that, if $\Sigma=S^{2}$, every value of $\boldsymbol{i} \in \mathfrak{I}_{4}(\Sigma)$ is realized, since (24.1) allows just one value, $\boldsymbol{i}=0$.

\section{A Diophantine equation}

Let $\Sigma$ be an oriented closed real surface, and let $M$ be the complex surface obtained by blowing up any set of $m$ distinct points in $\mathbf{C P}^{2}$, for $m=1, \ldots, 8$. Thus, $\mathcal{Z}(\Sigma, M)=\mathfrak{I}_{q}(\Sigma) \times \operatorname{Ker}\left[c_{1}(M)\right]$ (see Section 31). As a first step toward proving Theorem 2.17 for such $\Sigma$ and $M$, we will provide in this section an explicit description of the subset of $\mathcal{Z}(\Sigma, M)$ defined by the additional requirement (2.10). However, as $q=2$ ((f) in Section [27) and, by (2.9), $\mathfrak{I}_{2}(\Sigma)=\left\{w_{1}(\Sigma)\right\}=\{0\}$, the first component $\boldsymbol{i}$ of any element $(\boldsymbol{i}, \boldsymbol{d})$ of that subset is uniquely determined, so that we need only find the corresponding set of the second components $\boldsymbol{d}$. We use the identification (30.2) to treat $\boldsymbol{d}$ as a pair $(d, \boldsymbol{q})$ with $d \in \mathbf{Z}$ and $\boldsymbol{q} \in \mathbf{Z}^{m} \subset \mathbf{R}^{m}$ or, equivalently, as an $(m+1)$-tuple $\left(d, q_{1}, \ldots, q_{m}\right)$ of integers. We also set $\chi=\chi(\Sigma)$ and $\mathbf{1}=(1, \ldots, 1) \in \mathbf{R}^{m}$. The conditions imposed on $\boldsymbol{d}$ are $\boldsymbol{d} \in \operatorname{Ker}\left[c_{1}(M)\right]$ and $\boldsymbol{d} \cdot \boldsymbol{d}=-\chi$, which, by (30.3)-(30.4), amount to

$$
\langle\boldsymbol{q}, \mathbf{1}\rangle=3 d, \quad|\boldsymbol{q}|^{2}=d^{2}+\chi, \quad d \in \mathbf{Z}, \quad \boldsymbol{q} \in \mathbf{Z}^{m} .
$$

Here $\langle$,$\rangle is the inner product of \mathbf{R}^{m}$ and || is the corresponding norm.

We now proceed to solve equations (32.1) with $\chi \leq 2$ and $1 \leq m \leq 8$.

Example 32.1. Treating (32.1) as a system of equations imposed on $d$ and $\boldsymbol{q}=$ $\left(q_{1}, \ldots, q_{m}\right)$, in which $m, \chi \in \mathbf{Z}$ are fixed parameters with $m \geq 1$, we can rewrite it as $q_{1}+\ldots+q_{m}=3 d$ and $q_{1}^{2}+\ldots+q_{m}^{2}=d^{2}+\chi$, the unknowns now being $d, q_{1}, \ldots, q_{m} \in \mathbf{Z}$. Thus, since $q^{2} \equiv q \bmod 2$ for any $q \in \mathbf{Z}$, a solution to (32.1) exists only if $\chi$ is even. Each of the following three families of solutions to (32.1) represents infinitely many values of $m$ (with $d$ always denoting an integer):

(i) $(d, \boldsymbol{q})=(0, \mathbf{0})=(0,0, \ldots, 0)$, with any $m \geq 1$ and $\chi=0$,

(ii) $(d, \boldsymbol{q})=(d, \mathbf{1})=(d, 1, \ldots, 1)$, for $d \geq 1$, with $m=3 d$ and $\chi=(3-d) d$,

(iii) $(d, \boldsymbol{q})=(d, d-1,1, \ldots, 1)$, for any $d \geq 0$, with $m=2 d+2$ and $\chi=2$.

As we will show in Lemma 32.3 below, a solution to (32.1) with $\chi<0$ exists only if $m \geq 10$. In this regard, $m=10$ is a threshold value: solutions $(d, \boldsymbol{q})=$ $\left(d, q_{1}, \ldots, q_{m}\right)$ with $m=10$ not only exist for any prescribed even negative integer $\chi$, but can also be chosen so that $d>0$ and $q_{j}>0$ for all $j$, as illustrated by $(d, \boldsymbol{q})=(3 c+1+2 \varepsilon ; c, c, c, c+\varepsilon, \ldots, c+\varepsilon, 3)$, for integers $c \geq 2$ and $\varepsilon \in\{0,1\}$, with $\chi=8-2 \varepsilon-6 c$ or $(d, \boldsymbol{q})=(3 c+4 ; c+2, c, c+1, \ldots, c+1,3)$, for integers $c \geq 1$, with $\chi=4-6 c$.

Remark 32.2. Equations (32.1) remain satisfied after any permutation of the components $q_{1}, \ldots, q_{m}$ of $\boldsymbol{q}$, as well as after the signs of $d$ and all $q_{1}, \ldots, q_{m}$ have been changed. Also, a new solution with $m$ replaced by $m^{\prime}>m$ (or, $m^{\prime}<m$ ) arises if one inserts additional $m^{\prime}-m$ zeros (or, respectively, deletes existing $m-m^{\prime}$ 
zeros) among the $q_{1}, \ldots, q_{m}$. Successive applications of these operations, repeated in any order, any number of times, lead to what we call trivial modifications of the given solution $(d, \boldsymbol{q})$ to (32.1).

Lemma 32.3. No solutions to (32.1) exist when $\chi<0$ and $1 \leq m \leq 9$. If $\chi=0$, the only solution $(d, \boldsymbol{q})$ with $1 \leq m \leq 8$ is $(0, \mathbf{0})$, while the only solutions with $\chi=0$ and $m=9$ are $(3 s, s, s, s, s, s, s, s, s, s)$ for $s \in \mathbf{Z}$.

Proof. The Schwarz inequality $\langle\boldsymbol{q}, \mathbf{1}\rangle^{2} \leq|\boldsymbol{q}|^{2}|\mathbf{1}|^{2}$ becomes $(9-m) d^{2} \leq m \chi$ for $(d, \boldsymbol{q})$ with (32.1). If $1 \leq m \leq 9$, this gives $\chi \geq 0$. If, in addition, $\chi=0$, our Schwarz inequality yields $(9-m) d=0$, and so $\boldsymbol{q}$ is a multiple of $\mathbf{1}$ (the equality case in the Schwarz inequality). Thus, either $\chi=9-m=0$, or $m<9$ and $\chi=d=0$, which completes the proof.

For $(d, \boldsymbol{q}), m, \chi$ with (32.1) and the greatest integer $s$ with $3 s \leq d+1$,

$$
d=3 s+r, \quad \text { while } s \in \mathbf{Z} \text { and } r \in\{-1,0,1\} .
$$

Setting $\boldsymbol{s}=s \mathbf{1}=(s, \ldots, s) \in \mathbf{R}^{m}$ we now have $|\boldsymbol{q}|^{2}=d^{2}+\chi=9 s^{2}+6 r s+r^{2}+\chi$, $|\boldsymbol{s}|^{2}=m s^{2}$ and $\langle\boldsymbol{q}, \boldsymbol{s}\rangle=3 s d=3 s(3 s+r)=9 s^{2}+3 r s$. Hence

$$
|\boldsymbol{q}-\boldsymbol{s}|^{2}=(m-9) s^{2}+r^{2}+\chi \quad \text { and } r^{2} \in\{0,1\} .
$$

Lemma 32.4. The only solutions $(d, \boldsymbol{q})=\left(d, q_{1}, \ldots, q_{m}\right)$ to (32.1) with $\chi=2$ and $1 \leq m \leq 8$ are

$$
(0 ; 1,-1),(1 ; 1,1,1),(2 ; 1,1,1,1,1,1), \quad(3 ; 2,1,1,1,1,1,1,1),
$$

and those obtained from them by trivial modifications; cf. Remark 32.2 .

Note that, up to trivial modifications, (32.4) are precisely the solutions (iii) in Example 32.1 for $d=0,1,2,3$.

Proof. Let $(d, \boldsymbol{q})=\left(d, q_{1}, \ldots, q_{m}\right)$ satisfy (32.1) with $\chi=2$ and $1 \leq m \leq 8$. After a trivial modification we get

$$
d \geq 0 \quad \text { and } \quad q_{1} \ldots q_{m} \neq 0 .
$$

By (32.3), $s^{2} \leq(9-m) s^{2} \leq \chi+1=3$, and so $s \in\{-1,0,1\}$. Since $d \geq 0$, we have $s \in\{0,1\}$; cf. (32.2). For $\ell=|\boldsymbol{q}-\boldsymbol{s}|^{2}$, (32.3) with $\chi=2$ gives $\ell \in\{2,3\}$ (when $s=0$ ) or $\ell \in\{m-7, m-6\}$ (when $s=1$ ). As $\ell \geq 0$, it follows that $m \in\{6,7,8\}$ if $s=1$, while $m=\ell \in\{2,3\}$ and $\left|q_{1}\right|=\ldots=\left|q_{m}\right|=1$ if $s=0$, as $\ell=|\boldsymbol{q}|^{2} \leq 3$ and, by (32.5), $q_{1} \ldots q_{m} \neq 0$. The triple $(s, m, \ell)$ thus must assume one of the seven values $(0,2,2),(0,3,3),(1,6,0),(1,7,0),(1,7,1),(1,8,1)$ and $(1,8,2)$. When $s=1$, the relations $\ell=\sum_{j=1}^{m}\left(q_{j}-1\right)^{2} \leq 2$ and (32.5) imply that

$$
\ell \text { of the } m \text { integers } q_{1}, \ldots, q_{m} \text { equal } 2 \text {, and } m-\ell \text { of them equal } 1 \text {. }
$$

Thus, if $s=1$, we have $q_{1}+\ldots+q_{m}=m+\ell$, while, by (32.1),$q_{1}+\ldots+q_{m}=3 d$, and so $m+\ell=3 d$, which eliminates three of the seven triples $(s, m, \ell)$, leaving only those with $s=0$ or $m+\ell$ divisible by three: $(0,2,2),(0,3,3),(1,6,0)$, and $(1,8,1)$. These triples lead to the four possibilities listed in (32.4). Namely, $(0,2,2)$ and $(0,3,3)$ have $m \in\{2,3\}$ and $\left|q_{1}\right|=\ldots=\left|q_{m}\right|=1$, so that the sequence of two or three integers $q_{j}$ with values \pm 1 and sum $3 d \geq 0$ (cf. (32.1) and (32.5)) must be $( \pm 1, \mp 1)$ with $d=0$ or $(1,1,1)$ with $d=1$, as required. By (32.6), the triples $(1,6,0)$ and $(1,8,1)$ correspond in turn to the last two solutions in (32.4). 


\section{DEFORMATIONS OF PSEUDOHOLOMORPHIC IMMERSIONS}

We now present some facts needed in Section 34 to prove Theorem 2.18,

Given complex vector spaces $W, V$ and $A \in \operatorname{Hom}_{\mathbf{R}}(W, V)$, let $A^{ \pm}$stand for the unique operators $W \rightarrow V$ such that $A^{+}$is $\mathbf{C}$-linear, $A^{-}$is antilinear, and $A=A^{+}+A^{-}$. Thus, $A^{ \pm}=(A \mp i \circ A \circ i) / 2$ are the components of $A$ relative to the decomposition of $\operatorname{Hom}_{\mathbf{R}}(W, V)$ into the \pm 1 -eigenspaces of the involution $A \mapsto-i \circ A \circ i$, where $i$ stands for multiplication by $i$. Let $\nabla$ now be a fixed connection in a complex vector bundle $\eta$ over an almost complex manifold $\Sigma$. The Cauchy-Riemann operator $\bar{\partial}$ of $\nabla$ is the linear differential operator that takes any $C^{1}$ section $\psi$ of $\eta$ to

$$
\bar{\partial} \psi=[\nabla \psi]^{-},
$$

for [ $]^{-}$as in the preceding lines, so that $\bar{\partial} \psi$ is a section of $\operatorname{Hom}_{\mathbf{C}}(\overline{T \Sigma}, \eta$ ) (where $\overline{T \Sigma}$ is the complex conjugate bundle of $T \Sigma$ ) and its value $\bar{\partial} \psi_{x}$ at any $x \in \Sigma$ is $\left([\nabla \psi]_{x}\right)^{-}$. Note that $\nabla \psi$ itself is a section of $\operatorname{Hom}_{\mathbf{R}}(T \Sigma, \eta)$ sending $v \in T_{x} \Sigma$, for any $x \in \Sigma$, to $\nabla_{v} \psi \in \eta_{x}$. Thus, $\bar{\partial} \psi_{x}: T_{x} \Sigma \rightarrow \eta_{x}$ and $2 \bar{\partial} \psi_{x} v=\nabla_{v} \psi+i \nabla_{i v} \psi$ whenever $v \in T_{x} \Sigma$.

For a real $k$-dimensional manifold $\Sigma$ and an almost complex manifold $M$, let $[T M]^{\wedge k}$ denote the $k$ th complex exterior power of $T M$, and let $\operatorname{det}_{\mathbf{R}} T \Sigma$ be as in (5.2) ii). Any $C^{\infty}$ mapping $f: \Sigma \rightarrow M$ then gives rise to the vector bundle morphism $\operatorname{det} d f: \operatorname{det}_{\mathbf{R}} T \Sigma \rightarrow f^{*}\left([T M]^{\wedge k}\right)$ uniquely characterized by $(\operatorname{det} d f)_{x}\left(v_{1} \wedge \ldots \wedge v_{k}\right)=\left[d f_{x} v_{1}\right] \wedge \ldots \wedge\left[d f_{x} v_{k}\right]$ for $x \in \Sigma$ and $v_{1}, \ldots, v_{k} \in T_{x} \Sigma$. Obviously, $f$ is a totally real immersion if and only if $\operatorname{det} d f$ is nonzero everywhere as a section of $\operatorname{Hom}_{\mathbf{R}}\left(\operatorname{det}_{\mathbf{R}} T \Sigma, f^{*}\left([T M]^{\wedge k}\right)\right)$.

Given a pseudoholomorphic immersion $f$ of an oriented real surface $\Sigma$ in an almost complex surface $M$ (cf. the lines following Theorem 2.18) and a $C^{\infty}$ curve (homotopy) $I \ni t \mapsto f^{t}$ of mappings $\Sigma \rightarrow M$, where $I \subset \mathbf{R}$ is an interval, such that $0 \in I$ and $f^{0}=f$, let $\nu$ be the (complex) normal bundle of $f$ (see (5.1)). Then

$$
\text { i) }\left.\operatorname{det} d f^{t}\right|_{t=0}=0, \quad \text { ii) }\left.\frac{d}{d t} \operatorname{det} d f^{t}\right|_{t=0}=d f \wedge \bar{\partial} \psi .
$$

Here (i) reflects the fact that $f^{0}=f$ is pseudoholomorphic; (ii), however, requires further explanation. First, $\psi$ in (ii) is the section of $\nu$ obtained as the image of $\left(d f^{t} / d t\right)_{t=0}$ under the projection morphism $f^{*} T M \rightarrow \nu$, and $\bar{\partial}$ is the Cauchy-Riemann operator for a suitable connection in $\nu$, while both sides of (ii) are sections of $\operatorname{Hom}_{\mathbf{R}}\left(\operatorname{det}_{\mathbf{R}} T \Sigma, f^{*}\left([T M]^{\wedge 2}\right)\right)$ and $d f \wedge \bar{\partial} \psi$ is given by $(d f \wedge \bar{\partial} \psi)_{x}(u, v)=$ $\left[d f_{x} u\right] \wedge\left[\bar{\partial} \psi_{x} v\right]-\left[d f_{x} v\right] \wedge\left[\bar{\partial} \psi_{x} u\right]$ for $x \in \Sigma$ and $u, v \in T_{x} \Sigma$. Thus, with $i u \in T_{x} \Sigma$ defined using the $f$-pullback of the almost complex structure of $M$ to $\Sigma$,

$$
(d f \wedge \bar{\partial} \psi)_{x}(u, i u)=-2 i\left[d f_{x} u\right] \wedge\left[\bar{\partial} \psi_{x} u\right]
$$

due to complex-linearity of $d f_{x}$ and anti-linearity of $\bar{\partial} \psi_{x}$. Second, the spaces $\lambda_{x}=$ $\operatorname{Hom}_{\mathbf{R}}\left(\left[T_{x} \Sigma\right]^{\wedge 2},\left[T_{f(t, x)} M\right]^{\wedge 2}\right)$, with $f(t, x)=f^{t}(x)$, are the fibres of a complex line bundle $\lambda$ over $\Sigma \times I$, and $(x, t) \mapsto\left(\operatorname{det} d f^{t}\right)_{x}$ is, in view of (i), a section of $\lambda$, vanishing along the submanifold $\Sigma \times\{0\}$. In general, when a $C^{1}$ section $h$ of a vector bundle $\eta$ over a manifold $N$ vanishes at a point $y \in N$, one can define a linear operator $D h_{y}: T_{y} N \rightarrow \eta_{y}$ to be the composite in which the differential $d h_{y}: T_{y} N \rightarrow T_{(y, 0)} \eta$ (of $h$ treated as a mapping $N \rightarrow \eta$ into the total space) is 
followed by the projection $T_{(y, 0)} \eta \rightarrow \eta_{y}$ coming from the identifications $T_{(y, 0)} \eta=$ $T_{y} N \oplus T_{0} \eta_{y}$ and $T_{0} \eta_{y}=\eta_{y}$. (Hence, for $\eta=T^{*} N$ and $h=d \phi$ with any $C^{2}$ function $\phi: N \rightarrow \mathbf{R}$, the Hessian of $\phi$ at the critical point $y$ is $D h_{y}$.) We may now set $(d h(y(t)) / d t)_{t=0}=d h_{y} v$ whenever $t \mapsto y(t)$ is a $C^{1}$ curve in $N$ with $y(0)=0$, having the velocity $v \in T_{y} N$ at $t=0$. As a consequence of this for $\eta=\lambda$ and $N=\Sigma \times I$, along with any point $y=(x, 0)$ in the submanifold $\Sigma \times\{0\}$, and the curve $y(t)=(x, t)$, the left-hand side of (33.2)ii) is a well-defined section of $\operatorname{Hom}_{\mathbf{R}}\left(\operatorname{det}_{\mathbf{R}} T \Sigma, f^{*}\left([T M]^{\wedge 2}\right)\right)$.

Equality (33.2) ii) is now easily verified in local coordinates. For instance, one may fix a diffeomorphism between a neighborhood of any given point of $f(\Sigma)$ and a neighborhood $U$ of $(0,0)$ in $\mathbf{C}^{2}$, which makes $f$ appear as the inclusion $U \cap(\mathbf{C} \times\{0\}) \rightarrow \mathbf{C}^{2}$ and, at all points of $U \cap(\mathbf{C} \times\{0\})$, identifies the almost complex structure of $M$ with the standard complex structure of $\mathbf{C}^{2}$. The connection in $\nu$ is then defined only locally (as well as coordinate-dependent and nonunique), but the corresponding Cauchy-Riemann operator $\bar{\partial}$ is uniquely characterized by (33.3). A globally defined connection leading to $\bar{\partial}$ may now be obtained via a finite partition of unity.

\section{Proof of Theorem 2.18}

Lemma 34.1. Let $d x$ be a fixed positive smooth measure density on a compact manifold $\Sigma$ with $\operatorname{dim} \Sigma \geq 1$.

(i) For any finite-dimensional vector space $\mathcal{V}$ of real-valued continuous functions on $\Sigma$, there exist $C^{\infty}$ functions $f, h: \Sigma \rightarrow \mathbf{R}$, both $L^{2}$-orthogonal to $\mathcal{V}$, such that $|f|+|h|>0$ everywhere in $\Sigma$.

(ii) For any finite-dimensional vector space $\mathcal{W}$ of complex-valued continuous functions on $\Sigma$, some $C^{\infty}$ function $\varphi: \Sigma \rightarrow \mathbf{C}$ is $L^{2}$-orthogonal to $\mathcal{W}$ and nonzero everywhere in $\Sigma$.

Proof. To prove (i), set $k=\operatorname{dim} \mathcal{V}$, and let $\delta: \Sigma \rightarrow \mathcal{V}^{*}$ be the $C^{\infty}$ mapping assigning to each $x$ the evaluation functional (Dirac delta) $\delta[x]$ which sends $f$ to $f(x)$. Its image $\{\delta[x]: x \in \Sigma\}$ spans $\mathcal{V}^{*}$, as otherwise it would lie in a proper subspace; that is, some $f \in \mathcal{V} \backslash\{0\}$ would vanish at all $x \in \Sigma$. Thus, we may choose $2 k$ distinct points $x_{1}, \ldots, x_{k}, y_{1}, \ldots, y_{k} \in \Sigma$ such that both $\delta\left[x_{1}\right], \ldots, \delta\left[x_{k}\right]$ and $\delta\left[y_{1}\right], \ldots, \delta\left[y_{k}\right]$ are bases of $\mathcal{V}^{*}$, by first picking the $x_{a}$ and then selecting each $y_{a}$ near the corresponding $x_{a}$. Let us also fix pairwise disjoint open sets $U_{1}, \ldots, U_{k}, U_{1}^{\prime}, \ldots, U_{k}^{\prime}$ in $\Sigma$ with $x_{a} \in U_{a}$ and $y_{a} \in U_{a}^{\prime}$ for $a=1, \ldots, k$.

There must exist a $C^{\infty}$ function $f: \Sigma \rightarrow \mathbf{R}$ which is $L^{2}$-orthogonal to $\mathcal{V}$ and such that $f=1$ on $\Sigma \backslash U$, where $U=U_{1} \cup \ldots \cup U_{k}$. In fact, let $\phi_{1}, \ldots, \phi_{k}$ be the basis of $\mathcal{V}$ dual to the basis $\delta\left[x_{1}\right], \ldots, \delta\left[x_{k}\right]$ of $\mathcal{V}^{*}$. Thus, $\phi_{a}\left(x_{b}\right)=\delta_{a b}$ for $a, b=$ $1, \ldots, k$. The functions $\phi_{1}, \ldots, \phi_{k}$ are linearly independent when treated as linear functionals acting, via the $L^{2}$ inner product, on the space $\mathcal{F}$ of all $C^{\infty}$ functions $\Sigma \rightarrow \mathbf{R}$ with compact supports contained in $U$. (Otherwise, some nontrivial combination of the $\phi_{a}$, being $L^{2}$-orthogonal to $\mathcal{F}$, would vanish everywhere in $U$, which is impossible as $\phi_{a}\left(x_{b}\right)=\delta_{a b}$.) Hence, given $\lambda_{1}, \ldots, \lambda_{k} \in \mathbf{R}$, there exists $\xi \in \mathcal{F}$ with $\int_{\Sigma} \phi_{a} \xi d x=\lambda_{a}$ for $a=1, \ldots, k$. Choosing such $\xi$ for $\lambda_{a}=-\int_{\Sigma} \phi_{a} d x$, $a=1, \ldots, k$, we can now define $f$ by $f=\xi+1$.

The same argument may be applied to the $y_{a}$ and $U_{a}^{\prime}$ rather than $x_{a}$ and $U_{a}$. Thus, there exists a $C^{\infty}$ function $h: \Sigma \rightarrow \mathbf{R}$ which is $L^{2}$-orthogonal to $\mathcal{V}$ and 
such that $h=1$ on $\Sigma \backslash U^{\prime}$, with $U^{\prime}=U_{1}^{\prime} \cup \ldots \cup U_{k}^{\prime}$. As $(\Sigma \backslash U) \cup\left(\Sigma \backslash U^{\prime}\right)=\Sigma$, this yields (i). Now (ii) follows if we set $\varphi=f+i h$ with $f, h$ as in (i) for $\mathcal{V}=\{\operatorname{Re} \chi: \chi \in \mathcal{W}\}$.

Proof of Theorem 2.18. We have $f^{*}\left[\operatorname{det}_{\mathbf{C}} T M\right]=\tau \otimes \nu=\operatorname{Hom}(\bar{\tau}, \nu)$ as $f^{*} T M=$ $\tau \oplus \nu$, and so the equivalence of (a) and (b) is obvious. Furthermore, (c) implies (a) in view of (5.1) with $n=2$. Now assume (b) and let $\bar{\partial}$ be the Cauchy-Riemann operator appearing in (33.2) ii). Since $\bar{\partial}$ is elliptic, for any prescribed $C^{\infty}$ section $\phi$ of $\operatorname{Hom}(\bar{\tau}, \nu)$, solvability of the equation $\bar{\partial} \psi=\phi$ with an unknown $C^{\infty}$ section $\psi$ of $\nu$ is equivalent to $L^{2}$-orthogonality of $\phi$ to the kernel of the formal adjoint of $\bar{\partial}$. (To form the adjoint, one fixes Hermitian fibre metrics in $\tau$ and $\nu$, along with a positive smooth measure density on $\Sigma$.) Lemma 34.1(ii) now implies that $\phi$, for which the equation is solvable, may be chosen so as to be nowhere zero. (In fact, since we assume (b), we may fix a global $C^{\infty}$ section of $\operatorname{Hom}(\bar{\tau}, \nu)$, unit relative to the Hermitian fibre metric naturally determined by those in $\tau$ and $\nu$, and use it to treat sections of $\operatorname{Hom}(\bar{\tau}, \nu)$, including $\phi$, as functions $\Sigma \rightarrow$ C.) Choosing a deformation $t \mapsto f^{t}$ of $f$ in the direction of the corresponding solution $\psi$ and applying (33.2), we now obtain (c). Note that the section appearing in (33.2) ii) is nonzero everywhere in $\Sigma$, since the vectors within square brackets in (33.3), one tangent and one normal to $\Sigma$, are nonzero whenever $u \neq 0$.

\section{Proofs of Theorem 2.19 and Corollaries 2.20 - 2.21}

We begin by proving Theorem 2.19 in the special case where the $m$ distinct blown-up points, rather than being arbitrary, are selected in a particular way: the first $c$ of them lie in $\Sigma$, and the last $m-c$ in $M \backslash \Sigma$.

By blowing up the $m$ points we transform $\Sigma$ into a complex submanifold $\Sigma^{\prime}$ of the resulting complex surface $M^{\prime}$ with $\left[\Sigma^{\prime}\right]=([\Sigma], 1, \ldots, 1,0, \ldots, 0)$, where 1 occurs $c$ times. This is clear since the $\mathbf{Z}^{m}$ component of $[\Sigma]$ in the decomposition (2.11) is formed by intersection numbers of $\Sigma^{\prime}$ with the ordered $m$-tuple of exceptional divisors. Hence, by (30.1), the restriction of $c_{1}\left(M^{\prime}\right)$ to $\Sigma^{\prime}$ is zero, so that, in view of Theorem 2.18 the inclusion mapping $\Sigma^{\prime} \rightarrow M^{\prime}$ is homotopic to a totally real embedding $\hat{h}: \Sigma \rightarrow M^{\prime}$. (We identify $\Sigma^{\prime}$ with $\Sigma$ using the blow-down projection $\pi: M^{\prime} \rightarrow M$.) If $\hat{h}$ is chosen sufficiently $C^{1}$-close to the inclusion $\Sigma^{\prime} \rightarrow M^{\prime}$ (cf. Theorem 2.18),$\hat{h}(\Sigma)$ will have a single, transverse intersection with each of the first $c$ exceptional divisors and will not intersect the other $m-c$ of them. The composite $h=\pi \circ \hat{h}: \Sigma \rightarrow M$ thus is an embedding homotopic to the inclusion $\Sigma \rightarrow M$, having exactly $c$ complex points removable by blow-up (cf. Section 13), located at the first $c$ original blown-up points (that lay on $\Sigma$ ) and no other complex points.

Let $y_{1}, \ldots, y_{m}$ now be an arbitrary $m$-tuple of distinct points in $M$. Deforming $h$ slightly if necessary, we may assume that $y_{j} \notin h(\Sigma)$ for $j=1, \ldots, m$. (If some $y_{j}$ is one of the $c$ complex points of $h(\Sigma)$ removable by blow-up, we deform $h$ around $y_{j}$ using the flow of a $C^{\infty}$ vector field on $M$ supported in a small neigborhood of $y_{j}$, which is holomorphic near $y_{j}$, so that removability by blow-up is preserved.) Applying the final clause of Lemma 14.1 to these $h$ and $y_{j}$, we obtain an embedding $h^{\prime}: \Sigma \rightarrow M$ which, when the points $y_{1}, \ldots, y_{m}$ are blown up, becomes the required totally real embedding $f: \Sigma \rightarrow M^{\prime}$, completing the proof of Theorem 2.19. 
Proof of Corollary 2.20. This is just Theorem 2.19 with $c=3 d$ and $\Sigma$ realized as a nonsingular degree $d$ curve in $\mathbf{C P}^{2}$. (For instance, the curve given by $x^{d}+y^{d}+z^{d}=$ 0 in the homogeneous coordinates $[x, y, z]$.)

Proof of Corollary 2.21, If $d \leq 2$, the assertion follows from Corollary 2.20 for $d \leq 2$ (with a rearrangement of the blown-up points if $d=1$ ). Now let $d \geq 3$, and let $M$ be the complex surface obtained by blowing up a point in $\mathrm{CP}^{2}$. Using a complex automorphism of $\mathbf{C P}^{2}$, we may assume that the blown-up point is $[0,0,1]$. Equation $x^{d}=y^{d-1} z$, in the homogeneous coordinates $[x, y, z]$, defines a degree $d$ singular curve in $\mathbf{C P}^{2}$, and by blowing up its unique singularity, at $[0,0,1]$, we transform it into a nonsingular holomorphic curve $\Sigma \subset M$, diffeomorphic to $S^{2}$, with $[\Sigma]=(d, d-1)$. (In the holomorphic local coordinates $\xi, \eta$ for $M$ making the blow-down projection appear as $(\xi, \eta) \mapsto[\xi, \xi \eta, 1]$, equation $x^{d}=y^{d-1} z$ reads $\xi=\eta^{d-1}$, while the exceptional divisor is given by $\xi=0$.) We can now apply Theorem 2.19 to these $M, \Sigma$, with $c=2 d+1$ (cf. (30.1)) and $m=j-1$.

\section{The Remaining CaSes of Theorem 2.17}

In Section 31 we proved Theorem 2.17 except for two cases, which we deal with in this section. In the first remaining case, $\Sigma$ is assumed oriented and $M$ is obtained from $\mathrm{CP}^{2}$ by blowing up any set of $m$ distinct points, $1 \leq m \leq 8$. As shown in Lemmas 32.3 and 32.4 $\Sigma$ must then be either the torus $T^{2}$, with the degree $(0, \mathbf{0})$, or the sphere $S^{2}$, with one of the degrees (32.4) and their trivial modifications. (Cf. the beginnining of Section 32, ) We will now describe how each of these possibilities is realized.

For $(0, \mathbf{0})$, we fix a totally real 2 -torus in an open ball $U \subset \mathbf{C}^{2}$ (cf. (v) in Section (9) and then use a holomorphic embedding $U \rightarrow M$. That the degrees (32.4) and their trivial modifications are realized by totally real embedded 2-spheres with $m \leq 8$ is in turn clear, respectively, from Example 30.2 (combined with Lemma 12.1(a)), Corollary 15.3 for $m=3$ (along with Lemmas 12.1(a) and 32.4), and Corollary 2.21 for $d=2,3$.

To establish Theorem 2.17 for nonorientable surfaces $\Sigma$, which is the other remaining case, we first need an example and a lemma.

Given a simply connected almost complex surface $M$, a closed real surface $\Sigma$, and a fixed element $\boldsymbol{d}$ of $H_{2}\left(M, \mathbf{Z}_{[2]}\right)$ (notation of (2.3)), consider the following condition, imposed on $\boldsymbol{d}$, in which $q$ is defined by (1.4) and $\mathcal{Z}(\Sigma, M)$ is the set described in the lines following Theorem 2.2

$$
\text { Every }(\boldsymbol{i}, \boldsymbol{d}) \in \mathcal{Z}(\Sigma, M) \text { with the second component } \boldsymbol{d} \text { is the }
$$
index-degree pair of some totally real embedding $f: \Sigma \rightarrow M$.

Example 36.1. Condition (36.1) is satisfied by

(i) the torus $\Sigma=T^{2}$ and $M$ as above, with $\boldsymbol{d}=0$,

(ii) the Klein bottle $\Sigma=K^{2}$ and $\boldsymbol{d}=0$, with any $M$ as above for which $q=\infty$ or $q$ is finite but not divisible by 4 ,

(iii) $M=\mathbf{C P}^{1} \times \mathbf{C P}^{1}$ and $\Sigma=K^{2}$, with any $\boldsymbol{d} \in H_{2}\left(M, \mathbf{Z}_{2}\right) \backslash\{(1,1)\}$,

(iv) $M=\mathbf{C P}^{1} \times \mathbf{C P}^{1}$ and $\Sigma=S^{2}$, for $\boldsymbol{d}=( \pm 1, \mp 1) \in \mathbf{Z}^{2}=H_{2}(M, \mathbf{Z})$,

(v) $M=\mathbf{C P}^{2}$ and $\Sigma=\mathbf{R P}^{2}$, with $\boldsymbol{d}=1 \in\{0,1\}=\mathbf{Z}_{2}=H_{2}\left(M, \mathbf{Z}_{2}\right)$.

In fact, (i) and (ii) are immediate from (a)-(b) in Corollary 28.2. (iii) from Proposition 29.1, and (iv) from Remark 29.2 (where $\boldsymbol{i}$ must equal 0 by (24.1)). Finally, 
(v) follows since $\mathcal{Z}(\Sigma, M)=\mathfrak{I}_{6}\left(\mathbf{R} \mathrm{P}^{2}\right) \times \mathfrak{D}_{-}^{0}\left(\mathbf{C P}^{2}\right)$ has just one element (see (e) in Section 27, (24.3) and (24.4) ), and that element is realized by the totally real embedding described in (vi) of Section 9 .

In the following lemma, by the mod 2 reduction of $\boldsymbol{d} \in H_{2}\left(M, \mathbf{Z}_{2}\right)$ we mean d itself.

Lemma 36.2. Let $M$ be a fixed simply connected almost complex surface. If condition (36.1) holds for a closed real surface $\Sigma$ and $\boldsymbol{d} \in H_{2}\left(M, \mathbf{Z}_{[2]}\right)$, then (36.1) will remain satisfied after $\Sigma$ and $\boldsymbol{d}$ have been replaced by the connected sum $\Sigma \# T^{2} \# K^{2}$ and the $\bmod 2$ reduction of $\boldsymbol{d}$.

Proof. Let us assume (36.1), with our fixed $M$, for some given $\Sigma$ and $\boldsymbol{d}$. First, $M, \Sigma \# T^{2}$ and $\boldsymbol{d}$ then satisfy a modified version of (36.1), obtained when the word 'embedding' is replaced by the phrase immersion having just one double point, at which the self-intersection is transverse and, for orientable $\Sigma$, also negative in the sense of Section 16. This is clear from Example 36.1(i) applied, instead of $M$, to a simply connected open submanifold $U$ of $M \backslash \Sigma$, combined with Theorem 18.1 and (28.2). Specifically, to represent any given $(\boldsymbol{i}, \boldsymbol{d}) \in \mathcal{Z}\left(\Sigma \# T^{2}, M\right)$ with the second component $\boldsymbol{d}$ by a totally real immersion $f: \Sigma \# T^{2} \rightarrow M$ having a single self-intersection of the type just described, we write $\boldsymbol{i}=\left(\boldsymbol{i}_{*}, \mathbf{i}^{\prime}\right)$, as in (25.3) iii), and then obtain $f$ from the connected-sum operation performed on the totally real embeddings $\Sigma \rightarrow M$ and $T^{2} \rightarrow U \subset M$ that realize the index-degree pairs $\left(\boldsymbol{i}_{*}, \boldsymbol{d}\right)$ and $\left(\mathbf{i}^{\prime}, 0\right)$. (By (25.3), the additional condition imposed on elements of $\mathcal{Z}(\Sigma, M)$ or $\mathcal{Z}\left(\Sigma \# T^{2}, M\right)$ when $\Sigma$ is nonorientable and $\chi(\Sigma)$ is even, described in the lines following Theorem 2.2, holds for $(\boldsymbol{i}, \boldsymbol{d})$ if and only if it does for $\left(\boldsymbol{i}_{*}, \boldsymbol{d}\right)$.)

We now use Lemma 16.2 to remove the self-intersection of each totally real immersion $f: \Sigma \# T^{2} \rightarrow M$ obtained as above, which, as explained in Section 16, gives rise to a totally real embedding $\Sigma \# T^{2} \# K^{2} \rightarrow M$. Finally, the assertion about homotopy classes in Lemma 16.2 implies (36.1) for $\Sigma \# T^{2} \# K^{2}$ and $\boldsymbol{d}$. In fact, the connected-sum operation in Lemma16.2 resulting in $\Sigma \# T^{2} \# K^{2}$, may be viewed as involving one orientable summand: when $\Sigma$ is orientable, the summand in question is $\Sigma \# T^{2}$, while for nonorientable $\Sigma$ it is $T^{2}$ (since $\Sigma \# T^{2} \# K^{2}$ is then diffeomorphic to $\Sigma \# T^{2} \# T^{2}$ ). Thus, if $\Sigma$ is nonorientable, our claim follows from (25.3), since diffeomorphisms $T^{2} \rightarrow T^{2}$ equal to the identity on some disk in $T^{2}$ (and, therefore, admitting extensions to $\Sigma \# T^{2} \# T^{2}$ ) act on $H_{2}\left(T^{2}, \mathbf{Z}\right)=\mathbf{Z}^{2}$ so as to realize every automorphism in $\operatorname{SL}(2, \mathbf{Z})$. Consequently, the $T^{2}$ Maslov indices $\mathbf{Z}^{2} \rightarrow \mathbf{Z}_{q}$ constructed in Lemma 16.2 (which are trivial on one $\mathbf{Z}$ summand and arbitrary on the other) represent, after re-parametrization, all Maslov indices $\mathbf{Z}^{2} \rightarrow \mathbf{Z}_{q}$, as required. Similarly, for orientable $\Sigma$, we obtain our conclusion using diffeomorphisms of $K^{2}$ equal to the identity on a disk.

We will now prove the last remaining case of Theorem 2.17 in which $\Sigma$ is assumed nonorientable. First, let $M$ be $\mathbf{C}^{2}, \mathbf{C P}^{2}$ or $\mathbf{C P}^{1} \times \mathbf{C P}^{1}$. Every nonorientable closed surface admitting a totally real embedding in $M$ can now be obtained from just one or two low-genus primary surfaces by repeated applications of the operation $\Sigma \mapsto \Sigma \# T^{2} \# K^{2}$ (see Corollaries 2.12, 2.13 and 2.10). Specifically, for $M=\mathbf{C}^{2}$, or $M=\mathbf{C P}^{2}$, or $M=\mathbf{C P}^{1} \times \mathbf{C P}^{1}$, the primary surfaces are: $K^{2}$ alone, or $K^{2}$ and $\mathbf{R P}^{2}$ or, respectively, $K^{2}$ and $S^{2}$. The assertion of Theorem 2.17 for the complex surfaces $\mathbf{C}^{2}, \mathbf{C P}^{2}$ and $\mathbf{C P}^{1} \times \mathbf{C P}^{1}$, with all nonorientable $\Sigma$, is now immediate from Lemma 36.2, since, in view of Example 36.1, it holds for the 
primary surfaces just listed. (If $M=\mathbf{C}^{2}$ or $M=\mathbf{C P}^{2}$, (e) in Section 27 gives $q=\infty$ or $q=6$, and, in both cases, $\boldsymbol{d}=0$ for $\Sigma=K^{2}$ by (2.10) ii). Similarly, if $M=\mathbf{C P}^{1} \times \mathbf{C P}^{1},(2.10)$ ii) and (29.2) imply that $\boldsymbol{d} \neq(1,1)$ for $\Sigma=K^{2}$.)

Let $M$ now be obtained from $\mathbf{C P}^{2}$ by blowing up any set of $m \geq 1$ distinct points, and let $\Sigma$ be a totally real closed surface embedded in $M$. By the total mod 2 degree of $\Sigma$ we mean the pair $(d, s)$ with $d \in\{0,1\}$ and $s \in\{0,1, \ldots, m\}$, characterized as follows. The mod 2 reduction of the degree $[\Sigma] \in H_{2}\left(M, \mathbf{Z}_{[2]}\right)$ (equal to $[\Sigma]$ itself if $\Sigma$ in not orientable) can, in analogy with (30.2), be treated as an $(m+1)$-tuple $\left(d, q_{1}, \ldots, q_{m}\right)$ of elements of $\mathbf{Z}_{2}=\{0,1\}$. This gives the value of $d$, while $s$ is defined to be the number of times that $1 \in \mathbf{Z}_{2}$ occurs among the $q_{j}$. Thus, $s$ is the sum of the $q_{j}$ treated (and added) as integers with $q_{j} \in\{0,1\}$.

For $M, m, \Sigma, d, s$ with the properties just listed,

blowing up a point of $M$ that lies in $\Sigma$ replaces the quadruple $m, \Sigma, d, s$ with $m+1, \Sigma \# \mathbf{R P}^{2}, d, s+1$.

This is immediate from Lemma 12.1(b) for $n=k=2$ and Lemma 12.1(c).

If, in addition, $\Sigma$ is nonorientable or diffeomorphic to $S^{2}$, then

$$
d-s-\chi(\Sigma) \text { is divisible by } 4,
$$

by (2.10) with $\boldsymbol{d}^{2}=d-q_{1}-\ldots-q_{m}$, for $q_{j} \in\{0,1\} \subset \mathbf{Z}$ as above. The $S^{2}$ case follows if one applies the $\bmod 4$ reduction to (2.10) i), since $2=-2$ in $\mathbf{Z}_{4}$. We can rephrase (36.3) in the form of a table:

TABLE 2. The values, allowed by (36.3), of the total mod 2 degree $(d, s)$ for totally real embeddings with $1 \leq s \leq 7$ and the four 'primary' surfaces listed in the top row. Some values of $(d, s)$ for $S^{2}$ and for $s>7$ are listed as well.

\begin{tabular}{|c|c|c|c|c|c|}
\hline Row & $S^{2}$ & $\mathbf{R P}^{2}$ & $K^{2}$ & $K^{2} \# \mathbf{R} \mathbf{P}^{2}$ & $K^{2} \# K^{2}$ \\
\hline 1 & & & $(0,0)$ & $(0,1)$ & $(0,2)$ \\
\hline 2 & $(0,2)$ & $(0,3)$ & $(0,4)$ & $(0,5)$ & $(0,6)$ \\
\hline 3 & $(0,6)$ & $(0,7)$ & $(0,8)$ & $(0,9)$ & $(0,10)$ \\
\hline 4 & $(0,10)$ & $(0,11)$ & & & \\
\hline 5 & & $(1,0)$ & $(1,1)$ & $(1,2)$ & $(1,3)$ \\
\hline 6 & $(1,3)$ & $(1,4)$ & $(1,5)$ & $(1,6)$ & $(1,7)$ \\
\hline
\end{tabular}

Proving Theorem 2.17 for nonorientable surfaces is now reduced to realizing, for each row of Table 2 , the leftmost total mod 2 degree $(d, s)$ appearing in that row by a totally real embedding $\Sigma \rightarrow M$ (where $M$, again, arises from $\mathbf{C P}^{2}$ by blowing up $m$ points, and, this time, $m=s$ ). In fact, for the primary surfaces, the remaining total mod 2 degrees, in each row separately, are then immediately realized by starting from the leftmost one and then successively applying (36.2). Next, for all the remaining (nonprimary) nonorientable closed surfaces, Theorem 2.17 follows from Lemma 36.2 (which now states that realizability for $\Sigma$ implies the same for $\Sigma \# T^{2} \# K^{2}$, with $m$ and $(d, s)$ unchanged). On the other hand, Lemma 12.1(a) allows us to replace $m=s$ with any $m \geq s$.

The leftmost total mod 2 degree $(d, s)$ in each row is in turn realized as follows: Row 1, by Example 20.2 and Lemma 28.1. Row 2, in view of Example 30.2 Row 3 , from Corollary 2.21 for $d=2$ (and $j=6$ ); Row 4, by Corollary 2.21 for $d=4$ 
(and $j=10$ ); Row 5 , by (vi) of Section 9, and, finally, Row 6, from Corollary 2.20 for $d=1$ (and $m=3$ ).

The $m$ distinct blown-up points in the above argument can be made arbitrary, even though the left-to-right steps in every row of Table 2 use Lemma12.1, with the blown-up point lying on the original totally real surface. Namely, by Lemma12.1(c), after a small deformation each of the resulting totally real surfaces $\Sigma$ will have just one, transverse intersection point with any exceptional divisor that it intersects. Blowing down all such divisors gives rise to a finite set of complex points, all removable by blow-up, on a new surface $\Sigma^{\prime}$, and the final clause of Lemma 14.1 allows us to deform $\Sigma^{\prime}$ so as to move the complex points to arbitrarily prescribed locations, where they can be blown up again.

\section{Appendix. Spheres And tori in $\mathbf{C P}^{2} \# 9 \overline{\mathbf{C P}^{2}}$}

Our proof, in the last section, of Theorem 2.17 for nonorientable surfaces, works not only if $1 \leq m \leq 8$, but also for $m=9,10,11$. The question of extending the classification of Theorem 2.17 to $m \in\{9,10,11\}$ is thus reduced to determining which values of the degree are realized, for such $m$, by totally real embeddings of oriented closed surfaces.

Here we give a partial answer to this question for $m=9$. Namely, let $M=$ $\mathbf{C P}^{2} \# 9 \overline{\mathbf{C P}^{2}}$ be the complex surface obtained from $\mathbf{C P}^{2}$ by blowing up any ordered set of nine distinct points. By Lemma 32.3 , the only closed oriented surfaces admitting totally real embeddings in $M$ are $T^{2}$ and $S^{2}$. In the case of $T^{2}$, Lemma 32.3 also provides a list of possible degrees; in Example A.3 below we realize all those degrees by totally real embeddings. Theorem A.4 below provides, in turn, an "algebraic classification" of the degrees of totally real embeddings $S^{2} \rightarrow M$, analogous to that for $T^{2}$ in Lemma 32.3. We begin with some general remarks.

Since being totally real is an open property, a totally real immersion $f: \Sigma \rightarrow M$ with compact $\Sigma$ and $\operatorname{dim}_{\mathbf{R}} \Sigma<\operatorname{dim}_{\mathbf{C}} M$ leads, via a generic small deformation of $f$, to totally real embeddings. The following lemma shows that a similar deformation sometimes works when $\operatorname{dim}_{\mathbf{R}} \Sigma=\operatorname{dim}_{\mathbf{C}} M$.

Given a closed real manifold $\Sigma$, an integer $d \geq 2$, and a surjective homomorphism $\varphi: \pi_{1} \Sigma \rightarrow \mathbf{Z}_{d}$, let $\pi: \tilde{\Sigma} \rightarrow \Sigma$ be the $d$-fold covering projection corresponding to the subgroup $\operatorname{Ker} \varphi$ of $\pi_{1} \Sigma$. Thus, $\tilde{\Sigma}$ is a principal $\mathbf{Z}_{d}$ bundle over $\Sigma$, and we will denote by $\lambda$ the complex line bundle associated with it via the standard action of $\mathbf{Z}_{d}$ on $\mathbf{C}$. Note that $\lambda^{\otimes d}$ is trivial.

Lemma A.1. With $\Sigma, d, \varphi, \pi, \tilde{\Sigma}$ and $\lambda$ as above, let us suppose that $\lambda$ is realisomorphic to a vector subbundle of $T \Sigma$. Then, for any totally real embedding $f: \Sigma \rightarrow M$ in an almost complex manifold $M$, there exists a totally real embedding $\tilde{\Sigma} \rightarrow M$ which is $C^{\infty}$ homotopic, through totally real immersions, to the composite immersion $f \circ \pi$.

Proof. Since $f$ is totally real, our assumption about $\lambda$ allows us to choose an embedding $F: U \rightarrow M$, where $U$ is a neighborhood of the zero section $\Sigma$ in the total space of $\lambda$, such that $F=f$ on $\Sigma$. (In fact, we may let $F$ be the composite of an injective real vector-bundle morphism from $\lambda$ to the normal bundle of $f$, which exists in view of (5.1), followed by a tubular-neighborhood diffeomorphism.) As $\tilde{\Sigma}$ is naturally embedded in the total space of the unit circle bundle of $\lambda$ and $\pi: \tilde{\Sigma} \rightarrow \Sigma$ is the restriction to $\tilde{\Sigma}$ of the bundle projection $\pi: \lambda \rightarrow \Sigma$, the 
mappings given by $\tilde{\Sigma} \ni \xi \mapsto F(t \xi) \in M$, each of them depending on a fixed parameter $t \geq 0$ close to 0 , form a $C^{\infty}$ homotopy between $f \circ \pi$ (with $t=0$ ) and an embedding $\tilde{\Sigma} \rightarrow M$ (with any $t>0$ near 0 ). Being totally real is an open property; thus, for $t$ close to 0 such embeddings are totally real.

Example A.2. Given a totally real embedding $f: T^{2} \rightarrow M$ of the 2-torus in an almost complex surface $M$ and a $d$-fold self-covering $\pi: T^{2} \rightarrow T^{2}, d \geq 2$, there exists a totally real embedding $T^{2} \rightarrow M$ homotopic through totally real immersions to $f \circ \pi$. This is clear from Lemma A.1 for $\Sigma=\tilde{\Sigma}=T^{2}$. (The corresponding $\lambda$ is trivial, since so is $\lambda^{\otimes d}$ and $\pi_{1} \Sigma$ is free.)

Example A.3. Let $M=\mathbf{C P}^{2} \# 9 \overline{\mathbf{C P}^{2}}$ be the complex surface obtained by blowing up any set of nine distinct points in $\mathbf{C P}^{2}$. By Lemma 32.3, the degree of any totally real embedding $T^{2} \rightarrow M$ equals $(3 s, s, s, s, s, s, s, s, s, s)$ for some $s \in \mathbf{Z}$. We now show that, conversely, every such degree is realized by some totally real embedding $f: T^{2} \rightarrow M$. First, if $s=1$, the embedding $f$ is provided by Corollary 2.20 with $d=3$ and $m=9$. If $s>1$, we can use Example A.2 for this last $f$ and $d=s$. Finally, in the cases $s<0$ and $s=0$ it suffices to re-orient $\Sigma$ or, respectively, invoke Corollary 28.2 (a).

For the degrees of totally real embedded 2 -spheres, we have a partial result:

Theorem A.4. For every prescribed integer $d$, the system (32.1) with $m=9$ and $\chi=2$ has a unique solution $(d, \boldsymbol{q})=\left(d, q_{1}, \ldots, q_{m}\right)$ that satisfies the normalizing condition $q_{1} \geq \ldots \geq q_{m} ;$ cf. Remark 32.2. Explicitly, we have

$$
\left(q_{1}, \ldots, q_{9}\right)= \begin{cases}(s+1, s, s, s, s, s, s, s, s-1), & \text { if } d=3 s, s \in \mathbf{Z}, \\ (s+1, s+1, s+1, s, s, s, s, s, s), & \text { if } d=3 s+1, s \in \mathbf{Z}, \\ (s, s, s, s, s, s, s-1, s-1, s-1), & \text { if } d=3 s-1, s \in \mathbf{Z}\end{cases}
$$

In fact, (32.3) with $m=9$ and $\chi=2$ yields $|\boldsymbol{q}-\boldsymbol{s}|^{2} \in\{2,3\}$. Setting $p_{j}=$ $q_{j}-s$ and then decomposing 2 or 3 into all possible sums $\sum_{j=1}^{9} p_{j}^{2}$ with $p_{j} \in \mathbf{Z}$, $p_{1} \geq \ldots \geq p_{9}$ and $\sum_{j=1}^{9} p_{j}=3(d-3 s$ ) (which is the first equation in (32.1)), we easily obtain the required formula for $\left(q_{1}, \ldots, q_{9}\right)$.

\section{REFERENCES}

[1] A. Aeppli, On determining sets in a Stein manifold, Proc. Conf. Complex Analysis (Minneapolis, MN, 1964), 48-58, Springer, Berlin, 1965. MR0220975 (36:4027)

[2] V. I. Arnol'd, On a characteristic class entering into conditions of quantization (in Russian), Funkcional. Anal. i Priložen. 1 (1967), 1-14. MR0211415 (35:2296)

[3] P. Ahern and W. Rudin, Totally real embeddings of $S^{3}$ in $C^{3}$, Proc. Amer. Math. Soc. 94 (1985), 460-462. MR787894 (86g:32031)

[4] M. Audin, Fibrés normaux d'immersions en dimension double, points doubles d'immersions lagrangiennes et plongements totalement réels, Comment. Math. Helv. 63 (1988), 593-623. MR966952(89m:57032)

[5] E. Bedford, Invariant forms on complex manifolds with application to holomorphic mappings, Math. Ann. 265 (1983), 377-397. MR.721401 (85b:32046)

[6] V. Borrelli, On totally real isotopy classes, Internat. Math. Res. Not. 2002, 89-109. MR1874320 (2003a:57054) 
[7] Y. M. Eliashberg and V. M. Kharlamov, On the number of complex points of a real surface in a complex surface (in Russian), Proceedings of the Leningrad International Topological Conference (Leningrad, 1982), Nauka, Leningrad, 1983, 143-148. Zbl 0609.32016

[8] Y. Eliashberg and N. Mishachev, Introduction to the h-principle, Graduate Studies in Mathematics 48, AMS, Providence, RI, 2002. MR/1909245 (2003g:53164)

[9] T. Fiedler, Totally real embeddings of the torus into $\mathbf{C}^{2}$, Ann. Global Anal. Geom. 5 (1987), 117-121. MR944776 (89f:57043)

[10] F. Forstnerič, Some totally real embeddings of three-manifolds, Manuscripta Math. 55 (1986), 1-7. MR828408 (88f:32059)

[11] F. Forstnerič, On totally real embeddings into $C^{n}$, Exposition. Math. 4 (1986), 243-255. MR880125 (88g:32016)

[12] F. Forstnerič, A totally real three-sphere in $C^{3}$ bounding a family of analytic disks, Proc. Amer. Math. Soc. 108 (1990), 887-892. MR.1038758 (91g:32018)

[13] F. Forstnerič, Complex tangents of real surfaces in complex surfaces, Duke Math. J. 67 (1992), 353-376. MR1177310 (93g:32025)

[14] F. Forstnerič, Equivalence of real submanifolds under volume-preserving holomorphic automorphisms of $C^{n}$, Duke Math. J. 77 (1995), 431-445. MR1321065 (96b:32022)

[15] F. Forstnerič, Stein domains in complex surfaces, J. Geom. Anal. 13 (2003), 77-94. MR.1967038 (2004c:32050)

[16] F. Forstnerič and J. P. Rosay, Approximation of biholomorphic mappings by automorphisms of $C^{n}$, Invent. Math. 112 (1993), 323-349. MR1213106 (94f:32032)

[17] X. Gong, Unimodular invariants of totally real tori in $C^{n}$, American J. Math. 119 (1997), 19-54. MR1428057 (98f:32017)

[18] X. Gong, On totally real spheres in complex space, Math. Ann. 309 (1997), 611-623. MR.1483826 (99c:32023)

[19] M. Gromov, Convex integration of differential relations, I, Math. USSR, Izv. 7 (1973), 329343 (1974). MR0413206 (54:1323)

[20] M. Gromov, Partial Differential Relations, Ergebnisse, ser. 3, vol. 9, Springer-Verlag, BerlinNew York, 1986. MR.864505 (90a:58201)

[21] T. Kawashima, Some remarks on Lagrangian imbeddings, J. Math. Soc. Japan 33 (1981), 281-294. MR607945 (82e:53081)

[22] H.-F. Lai, Characteristic classes of real manifolds immersed in complex manifolds, Trans. Amer. Math. Soc. 172 (1972), 1-33. MR0314066 (47:2618)

[23] W. S. Massey, Pontryagin squares in the Thom space of a bundle, Pacific J. Math. 31 (1969), 133-142. MR0250332(40:3571)

[24] J. W. Milnor and J. D. Stasheff, Characteristic Classes, Annals of Mathematics Studies vol. 76, Princeton Univ. Press, Princeton, 1974. MR0440554 (55:13428)

[25] W. Rudin, Totally real Klein bottles in $\mathbf{C}^{2}$, Proc. Amer. Math. Soc. 82 (1981), 653-654. MR614897 (82i:32012)

[26] M. Slapar, Real surfaces in elliptic surfaces, Internat. J. Math. 16 (2005), 357-363. MR2133261 (2005k:32050)

[27] E. L. Stout and W. R. Zame, Totally real imbeddings and the universal convering spaces of domains of holomorphy: some examples, Manuscripta Math. 50 (1985), 29-48. MR.784138 (86h:32026)

[28] E. L. Stout and W. R. Zame, A Stein manifold topologically but not holomorphically equivalent to a domain in $C^{n}$, Adv. in Math. 60 (1986), 154-160. MR840302 (87i:32021)

[29] A. Weinstein, Lectures on symplectic manifolds. Corrected reprint, CBMS Regional Conference Series in Mathematics vol. 29, AMS, Providence, R.I., 1979. MR598470 (82b:58039)

[30] R. O. Wells, Compact real submanifolds of a complex manifold with non-degenerate holomorphic tangent bundles, Math. Ann. 179 (1969), 123-129. MR0237823 (38:6104)

[31] R. O. Wells, Function theory on differentiable submanifolds, Contributions to analysis (a collection of papers dedicated to Lipman Bers), 407-441. Academic Press, New York, 1974. MR0357856 (50:10322)

[32] H. Whitney, Topological properties of differentiable manifolds, Bull. Amer. Math. Soc. 43 (1937), 785-805. MR1563640

[33] H. Whitney, On the topology of differentiable manifolds, Lectures in Topology, pp. 101-141, University of Michigan Press, Ann Arbor, MI, 1941. MR0005300 (3:133a) 
[34] H. Whitney, The self-intersections of a smooth n-manifold in $2 n$-space, Ann. of Math. (2) 45 (1944), 220-246. MR0010274 (5:273g)

[35] H. Whitney, Classification of the mappings of a 3-complex in a simply connected space, Ann. of Math. (2) 50 (1949), 270-284. MR.0034020 (11:531c)

Department of Mathematics, The Ohio State University, Columbus, Ohio 43210

E-mail address: andrzej@math.ohio-state.edu

Department of Mathematics, The Ohio State University, Columbus, Ohio 43210 - And - Mathematical Institute, WrocŁaw University, 50-384 WrocŁaw, Poland - and Mathematical Institute, Polish Academy of Sciences, 51-617 Wroceaw, Poland

E-mail address: tjan@math.ohio-state.edu 